\title{
Multidisciplinary fall prevention : effects, feasibility and costs
}

Citation for published version (APA):

Hendriks, M. R. C. (2008). Multidisciplinary fall prevention : effects, feasibility and costs. [Doctoral Thesis, Maastricht University]. Datawyse / Universitaire Pers Maastricht. https://doi.org/10.26481/dis.20080611mh

Document status and date:

Published: 01/01/2008

DOI:

10.26481/dis.20080611mh

Document Version:

Publisher's PDF, also known as Version of record

\section{Please check the document version of this publication:}

- A submitted manuscript is the version of the article upon submission and before peer-review. There can be important differences between the submitted version and the official published version of record.

People interested in the research are advised to contact the author for the final version of the publication, or visit the DOI to the publisher's website.

- The final author version and the galley proof are versions of the publication after peer review.

- The final published version features the final layout of the paper including the volume, issue and page numbers.

Link to publication

\footnotetext{
General rights rights.

- You may freely distribute the URL identifying the publication in the public portal. please follow below link for the End User Agreement:

www.umlib.nl/taverne-license

Take down policy

If you believe that this document breaches copyright please contact us at:

repository@maastrichtuniversity.nl

providing details and we will investigate your claim.
}

Copyright and moral rights for the publications made accessible in the public portal are retained by the authors and/or other copyright owners and it is a condition of accessing publications that users recognise and abide by the legal requirements associated with these

- Users may download and print one copy of any publication from the public portal for the purpose of private study or research.

- You may not further distribute the material or use it for any profit-making activity or commercial gain

If the publication is distributed under the terms of Article $25 \mathrm{fa}$ of the Dutch Copyright Act, indicated by the "Taverne" license above, 
MULTIDISCIPLINARY FALL PREVENTION EFFECTS, FEASIBILITY AND COSTS 
ISBN: 9789052787305

Copyright, 2008, Marike RC Hendriks, Maastricht University, Department of Health Organization, Policy and Economics, Faculty of Health, Medicine and Life Sciences, PO Box 616, 6200 MD Maastricht, the Netherlands.

E-mail: marike.hendriks@beoz.unimaas.nl

University; School for Public Health and Primary Care (Caphri), Maastricht, Department of Clinical Epidemiology and Medical Technology Assessment, University Hospital Maastricht

Cover \& Titles: Esther van de Beek

Cover Illustration: Joost Rompa

Printed by Datawyse / Universitaire Pers Maastricht 


\title{
MULTIDISCIPLINARY FALL PREVENTION EFFECTS, FEASIBILITY AND COSTS
}

\author{
PROEFSCHRIFT \\ Ter verkrijging van de graad van doctor \\ aan de Universiteit Maastricht, \\ op gezag van de Rector Magnificus, \\ Prof. mr. G.P.M.F. Mols, \\ volgens het besluit van het College van Decanen, \\ in het openbaar te verdedigen \\ op woensdag 11 juni 2008 om 16.00 uur \\ door
} Marie Reinier Catherine Hendriks

$$
\begin{aligned}
& \text { U⿳⿵人一⿲丶丶㇒一 } \\
& \begin{array}{l}
\text { UNIVERSITAIRE } \\
\text { PERS MAASTRICHT }
\end{array}
\end{aligned}
$$


Promotores

Prof. dr. J.Th.M. van Eijk

Prof. dr. H.F.J.M. Crebolder

\section{Copromotores}

Dr. J.C.M. van Haastregt

Mr. dr. S.M.A.A. Evers

Beoordelingscommissie

Prof. dr. J.L. Severens (voorzitter)

Prof. dr. J.A. Knottnerus

Prof. dr. J.M.G.A. Schols

Prof. dr. H.J.J. Verhaar (Universitair Medisch Centrum Utrecht)

Prof. dr.ir. H.C.W. de Vet (Vrije Universiteit Amsterdam)

The Netherlands Organization for Health Research and Development (ZonMw, grant number 945-02-053) is gratefully acknowledged for funding the studies in this thesis. 


\section{CONTENTS}

1 General Introduction $\quad 7$

2 A multidisciplinary fall prevention programme for elderly per- 21 sons: a feasibility study

3 Effectiveness and cost-effectiveness of a multidisciplinary intervention programme to prevent new falls and functional decline among elderly persons at risk: design of a replicated randomised controlled trial [ISRCTN64716113]

4 Lack of effectiveness of a multidisciplinary fall prevention 53 programme in elderly people at risk: a randomized controlled trial [ISRCTN64716113]

5 Process factors explaining the ineffectiveness of a multidiscipli- 73 nary fall prevention programme

6 Cost-effectiveness of a multidisciplinary fall prevention programme in community-dwelling elderly people: a randomized controlled trial [ISRCTN64716113]

7 Discontinuous cost measurement: how to fill in the gaps?

8 General discussion

Summary

Samenvatting

Co-authors \& Affiliations 155

Dankwoord 157

Curriculum Vitae 

INTRODUCTION | 7

\section{INTRODUCTION}

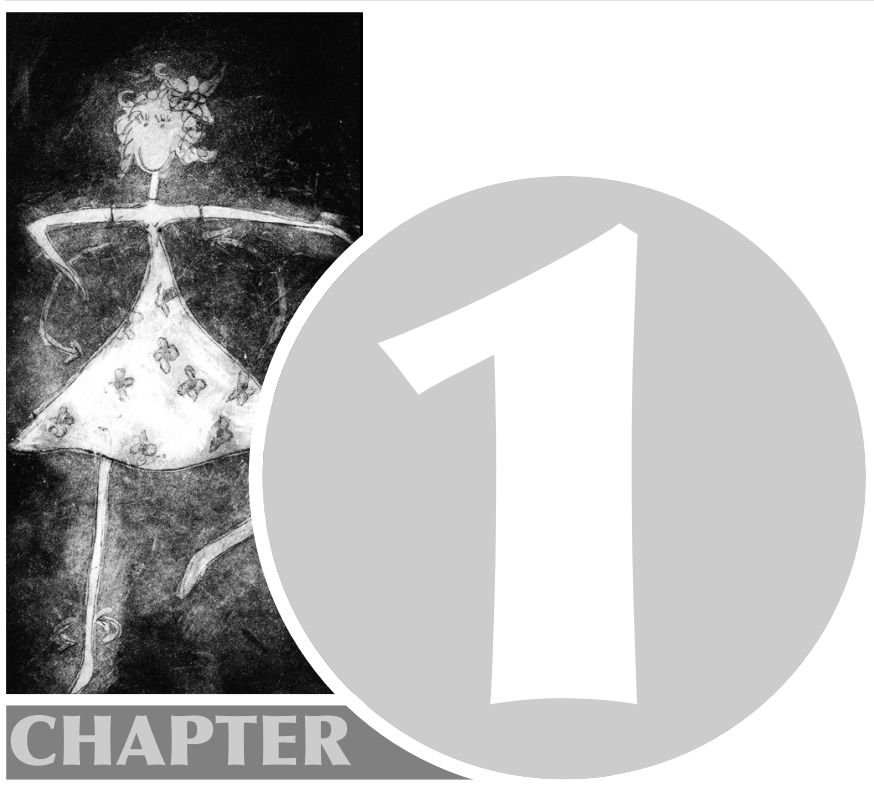





\section{FALLS}

Everyone has probably experienced a fall at some time in their life. In fact, falls are common occurrences for most people. When children learn to walk, they usually fall frequently. The process of learning various new physical skills and sports is often accompanied by falls. Generally, when persons slip or trip and fall, they get up quickly and walk on, sometimes being somewhat embarrassed, but sooner or later they forget the fall they have experienced. In elderly people, however, falls often have serious consequences. Many older people are unable to get up by themselves after a fall. ${ }^{1,2}$ Generally, as people grow older, the risk of falls increases and the consequences often become worse. $^{3}$

The present thesis describes a study on fall prevention in community-living elderly people at risk for falling. In this thesis, a fall is defined as an event that results in a person coming to rest inadvertently on the ground or other lower level. ${ }^{4}$ The following sections describe the incidence and consequences of falls among elderly people, the current state of knowledge about the aetiology of falls, the prevention of falls, and the importance of economic evaluations in fall prevention. Finally, the aims of the present study are discussed and an outline of the thesis is provided.

\section{INCIDENCE AND CONSEQUENCES OF FALLS}

Falls are a common problem among elderly people. About one-third of community-living people aged 65 years and over fall at least once a year ${ }^{3,5-9}$. This fall rate increases with age. ${ }^{3,5,7-10}$ Moreover, people who have sustained a fall are at greater risk of falling again. ${ }^{6,9,11-13}$ Falls in elderly people may have several adverse consequences for both the individual and the community.

Although most falls do not lead to serious injury, ${ }^{2}$ around $40 \%$ to $70 \%$ result in some kind of physical injury, varying from minor to major, for example soft tissue injuries (e.g. bruises or scrapes), contusions, lacerations, sprains, head injuries and joint dislocations. ${ }^{2,10,13-15}$ About $5 \%$ to $10 \%$ of the falls among community-living older persons result in fractures, including hip fractures. ${ }^{2,3,7,9,14,16,17}$ Hip fractures and traumatic brain injury are the most serious fall injuries. ${ }^{18,19}$ Injuries resulting from falls may cause temporary or perma- 
nent mobility decline, and/or decline in daily functioning. ${ }^{2,5,13,15,16}$ Falls can also have psychosocial consequences, such as fear of falling, avoidance of activity, decreased self-efficacy, loss of confidence, loss of independence and a decreased quality of life..$^{2,3,15,17,19-24}$ Between $30 \%$ and $73 \%$ of people who experience an accidental fall develop fear of falling., ${ }^{3,20,22,26}$ People who are afraid to fall, often express a low confidence in their ability to engage in certain activities without falling, and therefore regularly restrict activities that they could engage in safely., ${ }^{3,-23,25-29}$ Subsequently, activity avoidance may lead to physical inactivity, loss of independence or social isolation. ${ }^{28,29}$ Several studies have confirmed that fear of falling is associated with decreased quality of life, increased frailty, depressed mood, lower mobility and functional decline. ${ }^{3,17,22,24-27}$ From the perspective of elderly people, it is evident that falls should be prevented as much as possible, as was illustrated by Salkeld and colleagues, ${ }^{19}$ who reported that $80 \%$ of their respondents would rather be dead than experience the loss of independence and quality of life that results from a bad hip fracture and subsequent admission to a nursing home.

In addition to consequences for individuals, falls also have a societal impact, especially due to the additional use of healthcare resources. One-fifth of all falls necessitate medical attention for some kind of injury. ${ }^{6,14,15,17,30}$ Injurious falls in particular are related to increased morbidity, which subsequently may cause increased healthcare utilization, resulting in excessive healthcare costs. ${ }^{2,5,7,14,18,31-35}$ In terms of the diagnostic groups accounting for the highest healthcare expenditures in the Netherlands, falls rank third (accounting for $5.9 \%$ of the healthcare costs) after dementia and stroke among the oldest elderly (>85) and tenth (accounting for $2.5 \%$ of the healthcare costs) among those aged between 65 and 84 years. ${ }^{35,36}$ Hip fractures particularly contribute to fall-related health expenditures, ${ }^{37}$ for example due to admission to hospital or a nursing home. ${ }^{3,8,17,19,38}$ Falls have been recorded as a contributing factor in $40 \%$ of nursing home admissions. ${ }^{9}$ Recent studies confirm that falls are associated with substantial costs. ${ }^{35-37,39-43}$ The burden and costs of falls and fallinduced injuries are expected to rise further in the coming decades, because of the growing number of elderly persons resulting from the post-war baby boom. ${ }^{17,44}$ In view of the individual as well as societal impact of falls, the need for effective fall prevention strategies is evident. 


\section{AETIOLOGY OF FALLS}

In search of effective prevention strategies, various causes or risk factors for falls in community-living elderly people have been identified. ${ }^{3,6,7,9,10,14,45-49}$ Risk factors for falls can be roughly subdivided into intrinsic risk factors (individual, activity-related, health or predisposing factors) and extrinsic risk factors (situational or environmental factors). ${ }^{3,9,14,16,49}$ Examples of intrinsic risk factors are mobility impairments like muscle weakness, impaired balance or gait or impaired activities of daily living, while examples of extrinsic risk factors are slip and trip hazards, like loose carpets or wet floors but also inappropriate footwear. The literature provides no unequivocal list of risk factors contributing to the incidence of falls, partly because studies into the aetiology of falls often differ considerably in population, definitions of risk factors, methodology and statistical analyses. ${ }^{13,50}$ Table 1 presents, in alphabetical order, an overview of intrinsic and extrinsic risk factors which are considered to contribute to the incidence of falls in community-living elderly people.

Some risk factors cause falls directly (e.g. muscle weakness), but others can be considered markers of underlying causes (e.g. advanced age). ${ }^{3}$ As people grow older and become more frail, they often suffer simultaneously from several diseases and impairments. ${ }^{44}$ As a result, various risk factors for falling are present simultaneously in these elderly persons. Furthermore, they become increasingly susceptible to environmental hazards due to the accumulating effects of age and disease, impairments and disabilities. $3,7,13,14,48,49,51,52$ Although some falls are caused by a single factor, most falls are complex events caused by the interaction between several risk factors. ${ }^{3,7,14,49}$ The risk of falls increases with the number of risk factors present, ${ }^{2,3,7,48,49}$ and the presence of risk factors for falls in itself may increase the risk of developing additional risk factors. Consequently, frail elderly persons may enter a debilitating spiral of falls, fear of falling and functional decline..$^{26,27,53}$ 
Table 1 Risk factors for falls in community-living elderly people

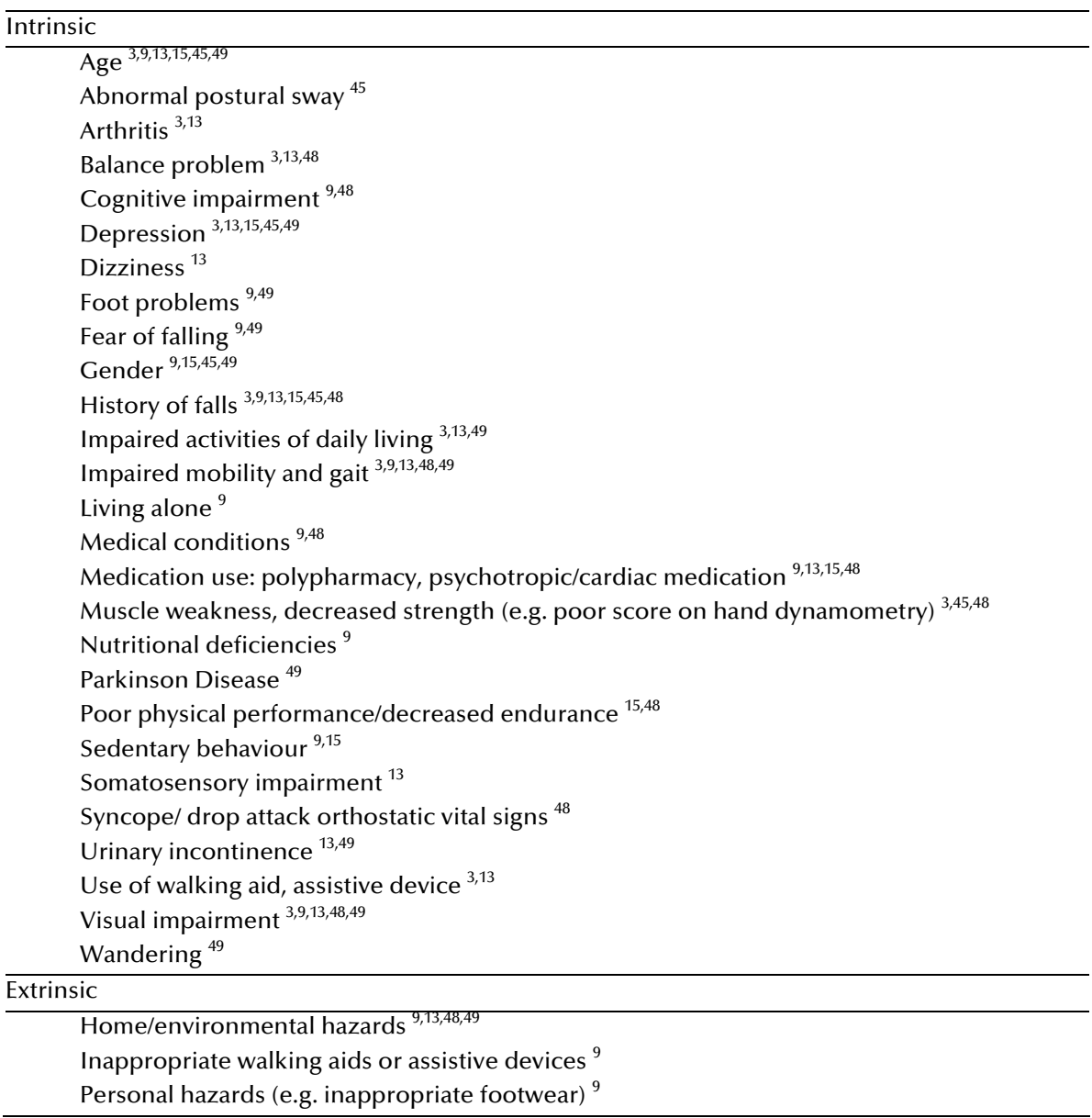

\section{PREVENTION OF FALLS}

Many risk factors for falls can potentially be treated or modified, 3,13,14,49,51 helping to prevent future falls. Fall prevention programmes therefore aim to treat or modify possible risk factors for falls, some being designed to reduce the impact of a single factor, others aiming at multiple risk factors. ${ }^{6}$ Different categories of fall prevention programmes described in the literature are: cognitive/behavioural interventions, cardiac pacemaker insertions, exercise/physical therapy programmes, home hazard/environmental assessment and modification, medication withdrawal/adjustment, nutritional/vitamin supplementation, pharmacological therapies, referral for visual deficiencies, 
and multidisciplinary, multifactorial intervention programmes. ${ }^{6,8,14}$ Multidisciplinary and multifactorial fall prevention programmes often consist of a postfall assessment or systematic risk factor screening, followed by recommendations and follow-up for risks uncovered ${ }^{8}$. Recent reviews confirm that multidisciplinary and multifactorial interventions that screen for health as well as environmental risk factors and address these factors are most likely to be effective in preventing falls. ${ }^{6,8,14}$ This seems to be a logical consequence of the fact that falls are often caused by interactions between several risk factors. ${ }^{8,17,18,25,54}$ Nevertheless, the effects of these interventions are generally small and there is some inconsistency in the results of different trials in this domain. ${ }^{3,6}$

The strongest evidence for effective fall prevention is found when multifactorial patient-centred interventions aim at a high-risk population, for example people who have sustained an injurious fall. ${ }^{8,955}$ Close and colleagues ${ }^{11}$ developed such a multifactorial programme aimed at a high-risk group: community-living elderly persons attending the Accident and Emergency (A\&E) department after an injurious fall. The programme, consisting of a medical and occupational-therapy assessment, was evaluated in the inner city of London (United Kingdom), and showed promising results in terms of preventing falls and functional decline. ${ }^{11}$ However, it is obvious that when a programme appears to be effective in one healthcare setting in a particular country, this is no guarantee that it will also be effective in another healthcare setting in other countries, so additional research is needed to confirm the effectiveness of the intervention in other healthcare settings in other countries.

In the Netherlands, there is currently no clear evidence for the effectiveness of multifactorial intervention programmes. Moreover, at the start of this research project, there were no guidelines on preventing falls in the Netherlands. ${ }^{56}$ Although there is much debate about fall prevention, there is no standard approach to fall risk assessment and management for older fallers who present to the A\&E department and are discharged home. ${ }^{13}$ No systematic attention is generally given in the Netherlands to the specific consequences of injurious falls for the daily functioning of individual patients in their unique situation. We therefore decided to evaluate the above successful British programme developed by Close and colleagues ${ }^{11}$ in a randomized controlled trial (RCT) in the Netherlands, to assess whether the effectiveness of the programme could also be established in the Dutch healthcare setting. 


\section{ECONOMIC EVALUATION OF FALL PREVENTION}

As stated above, the financial burden of fall-related injuries is significant. ${ }^{18,35-}$ 37,39-43 In the last decade, some economic evaluations of fall prevention programmes have been performed, but these studies were limited in their assessment of healthcare costs, did not include all healthcare and patient family costs, or studied other intervention programmes. ${ }^{11,57-64}$ Although fall prevention programmes initially require investments, such programmes may preserve people's autonomy and independence, and therefore result in a reduction of total long-term healthcare costs. Furthermore, if a fall prevention programme leads to a reduction in injurious falls and fractures and subsequent healthcare utilization, it is likely to be cost-effective. However, the cost savings achieved by multidisciplinary and multifactorial interventions remain unclear. ${ }^{9,17}$ Additional research is therefore needed to ascertain whether these multidisciplinary and multifactorial fall prevention strategies are costeffective. ${ }^{6,9,17,35,38,54}$ Moreover, policymakers and decision makers need information about cost-effectiveness to enable them to decide about implementing an intervention programme. Therefore, we also planned an economic evaluation to assess the cost-effectiveness of the multidisciplinary intervention programme to prevent falls in the Dutch healthcare setting, from a broad societal perspective.

Nowadays, healthcare interventions studies (not only those on fall prevention) include economic evaluations to identify the most cost-effective alternative. Economic evaluations are preferably performed from a societal perspective, which means that participants have to report their healthcare utilization continuously. ${ }^{65,66}$ Because this often represents a burden to the participants, often resulting in drop-outs and missing values, discontinuous measurement is increasingly being used (e.g. measuring during three months a year). ${ }^{67-69}$ Although previous studies have established the reliability of this method, ${ }^{65,70}$ it remains unclear what is the best way to estimate annual costs from these periodic measurements. This was another topic we wanted to address within our economic evaluation study. We therefore compared continuous measurements with discontinuous measurements. 


\section{AIMS AND OUTLINE OF THIS THESIS}

\section{Aims of this study}

In the Netherlands, the larger part of healthcare costs relate to care, rather than to cure. ${ }^{36}$ In view of this, it seems important to prevent falls in order to preserve long-term autonomy and quality of life among elderly people, and consequently prevent long-term costs of care. This is in accordance with the Dutch government's policy and laws, such as the Social Support Act that came into force in the Netherlands in 2007. This act promotes participation in society and independent functioning and living in the community. ${ }^{71}$ If falls and functional decline can be prevented in community-living elderly people, they can continue to live independently and preserve their autonomy. The target group of our study therefore consisted of elderly people at risk for falling who were living independently in the community.

This thesis describes a randomized controlled trial (RCT) evaluating the effectiveness, feasibility and cost-effectiveness of a multidisciplinary programme to prevent falls and functional decline in elderly people in the Netherlands. The programme was based on a British programme developed by Close and colleagues, ${ }^{11}$ which showed promising effects on falls and daily functioning. The programme comprised a medical and occupational-therapy assessment followed by referrals and/or recommendations if indicated, and was aimed at a high-risk group: community-living elderly persons attending an A\&E department after an injurious fall. We adapted the programme to the Dutch situation to assess its robustness and to ascertain whether it could be recommended for implementation in regular healthcare the Netherlands.

The main objective of this thesis is therefore to explore the effectiveness, feasibility and cost-effectiveness of the medical and occupational-therapy programme to prevent falls and functional decline in elderly people at risk, compared to usual healthcare in the Netherlands. This thesis describes five studies, whose objectives are summarized below.

1. To develop a Dutch version of a successful British multifactorial intervention programme to prevent falls among elderly people.

2. To assess the effectiveness of the adapted version of this fall prevention programme in the Netherlands in terms of falls, functional decline and a number of secondary outcomes. 
3. To assess the feasibility of the adapted Dutch version of the fall prevention programme for elderly persons and implementers.

4. To assess the cost-effectiveness of the adapted version of the fall prevention programme in the Netherlands, from a broad societal perspective.

5. To assess the best way to calculate annual costs when health expenditures are measured discontinuously.

\section{Outline of this thesis}

Chapter 2 describes the process of adapting the British intervention programme to prevent falls and the pilot study in which we assessed the feasibility of the adapted programme in Dutch healthcare. After the pilot study, a larger randomized controlled trial (RCT) was planned to assess the effectiveness and cost-effectiveness of the fall prevention programme. Chapter 3 presents the design of this two-group RCT with twelve months of follow-up, comprising an effect evaluation, a process evaluation and an economic evaluation. Details about recruitment of the study population are given, followed by a short description of the intervention and control programmes. Objectives as well as outcome measures are described for each of the three evaluations. Chapter 4 reports on our evaluation of the programme's effectiveness versus usual healthcare in the Netherlands, offering extensive analyses and results in terms of primary and secondary outcome measures. Chapter 5 presents the process evaluation of the programme which aimed to assess the feasibility of the programme. Chapter 6 describes the costeffectiveness evaluation including detailed descriptions of the health expenditures related to the programme compared with usual care. Chapter 7 reports a study to assess the best way to calculate annual costs from discontinuously measured health expenditures. We compared several discontinuous measurement patterns with continuous measurement, combining the discontinuous patterns with three imputation techniques: Individual Mean (IM), Last Observation Carried Forward (LOCF) and Next Observation Carried Backward (NOCB). The final chapter reflects on the major findings of this thesis, discusses its strengths and limitations and offers implications and recommendations for future fall prevention practice as well as fall prevention research. Since chapters 2 to 7 of this thesis are based on articles published in scientific journals, some overlap between them is inevitable. 


\section{REFERENCES}

1. Tinetti ME, Liu WL, Claus EB. Predictors and prognosis of inability to get up after falls among elderly persons. JAMA 1993;269(1):65-70.

2. Nevitt MC, Cummings SR, Hudes ES. Risk factors for injurious falls: a prospective study. J Gerontol 1991;46(5):M164-70.

3. Rubenstein LZ. Falls in older people: epidemiology, risk factors and strategies for prevention. Age Ageing 2006;35 Suppl 2:ii37-ii41.

4. The prevention of falls in later life. Dan Med Bull 1987;34 Suppl 4:1-24.

5. Blake AJ, Morgan K, Bendall MJ, et al. Falls by elderly people at home: prevalence and associated factors. Age Ageing 1988;17(6):365-72.

6. Gillespie LD, Gillespie WJ, Robertson MC, Lamb SE, Cumming RG, Rowe BH. Interventions for preventing falls in elderly people. Cochrane Database Syst Rev 2003(4):CD000340.

7. Tinetti ME, Speechley M, Ginter SF. Risk factors for falls among elderly persons living in the community. N Engl J Med 1988;319(26):1701-7.

8. Chang JT, Morton SC, Rubenstein LZ, et al. Interventions for the prevention of falls in older adults: systematic review and meta-analysis of randomised clinical trials. BMJ 2004; 328(7441):680.

9. Todd C, Skelton D. What are the main risk factors for falls among older people and what are the most effective interventions to prevent falls? Copenhagen: WHO Regional Office for Europe (Health Evidence Network), 2004: 1-28.

10. Masud T, Morris, R.O. Epidemiology of falls. Age Ageing 2001;30 (S4):3-7.

11. Close J, Ellis M, Hooper R, Glucksman E, Jackson S, Swift C. Prevention of falls in the elderly trial (PROFET): a randomised controlled trial. Lancet 1999;353(9147):93-97.

12. Nevitt MC, Cummings, S.R., Kidd, S., Black, D. Risk factor for recurrent nonsyncopal falls. JAMA 1989;261:2663-2668.

13. Russell MA, Hill KD, Blackberry I, Day LL, Dharmage SC. Falls risk and functional decline in older fallers discharged directly from emergency departments. J Gerontol A Biol Sci Med Sci 2006;61(10):1090-5.

14. Kannus $P$, Sievanen $H$, Palvanen $M$, Jarvinen T, Parkkari J. Prevention of falls and consequent injuries in elderly people. Lancet 2005;366(9500):1885-93.

15. Stel VS, Smit JH, Pluijm SM, Lips P. Consequences of falling in older men and women and risk factors for health service use and functional decline. Age Ageing 2004;33(1):58-65.

16. Fuller GF. Falls in the elderly. Am Fam Physician 2000;61(7):2159-68, 2173-4.

17. Kannus $P$, Khan KM. Prevention of falls and subsequent injuries in elderly people: a long way to go in both research and practice. CMAJ 2001;165(5):587-88.

18. Stevens JA. Falls among older adults--risk factors and prevention strategies. J Safety Res 2005; 36(4):409-11.

19. Salkeld G, Cameron ID, Cumming RG, et al. Quality of life related to fear of falling and hip fracture in older women: a time trade off study. BMJ 2000;320(7231):341-6.

20. Jorstad EC, Hauer K, Becker C, Lamb SE. Measuring the psychological outcomes of falling: a systematic review. J Am Geriatr Soc 2005;53(3):501-10.

21. Zijlstra GA, van Haastregt JC, van Eijk JT, van Rossum E, Stalenhoef PA, Kempen GI. Prevalence and correlates of fear of falling, and associated avoidance of activity in the general population of community-living older people. Age Ageing 2007;36(3):304-9.

22. Vellas BJ, Wayne SJ, Romero LJ, Baumgartner RN, P.J. G. Fear of falling and restriction of mobility in elderly fallers. Age Ageing 1997;26:189-193.

23. Vellas B, Cayla F, Bocquet H, de Pemille F, Albarede JL. Prospective study of restriction of activity in old people after falls. Age Ageing 1987;16(3):189-93. 
24. Arfken CL, Lach HW, Birge SJ, Miller JP. The prevalence and correlates of fear of falling in elderly persons living in the community. Am J Public Health 1994;84(4):565-70.

25. Tinetti M, Doucette JT, Claus EB. The contribution of predisposing and situational risk factors to serious fall injuries. J Am Geriatr Soc 1995;43:1207-13.

26. Yardley L, Smith H. A prospective study of the relationship between feared consequences of falling and avoidance of activity in community-living older people. Gerontologist 2002;42(1):1723.

27. Friedman SM, Munoz B, West SK, Rubin GS, Fried LP. Falls and fear of falling: which comes first? A longitudinal prediction model suggests strategies for primary and secondary prevention. J Am Geriatr Soc 2002;50(8):1329-35.

28. Murphy SL, Dubin JA, Gill TM. The development of fear of falling among community-living older women: predisposing factors and subsequent fall events. J Gerontol A Biol Sci Med Sci 2003;58(10):M943-7.

29. Delbaere K, Crombez G, Van Den Noortgate N, Willems T, Cambier D. The risk of being fearful or fearless of falls in older people: an empirical validation. Disabil Rehabil 2006;28(12):751-6.

30. Bergland A, Wyller TB. Risk factors for serious fall related injury in elderly women living at home. Inj Prev 2004;10(5):308-13.

31. Speechley $M$, Tinetti $M$. Falls and injuries in frail and vigorous community elderly persons. $J$ Am Geriatr Soc 1991;39(1):46-52.

32. Sattin RW, Rodriguez JG, DeVito CA, Wingo PA. Home environmental hazards and the risk of fall injury events among community-dwelling older persons. J Am Geriatr Soc 1998;46:669-76.

33. Kiel DP, O'Sullivan P, Teno JM, Mor V. Health care utilization and functional status in the aged following a fall. Med Care 1991;29(3):221-8.

34. Rizzo JA, Friedkin R, Williams CS, Nabors J, Acampora D, Tinetti ME. Health care utilization and costs in a Medicare population by fall status. Med Care 1998;36(8):1174-88.

35. Meerding WJ, Mulder S, van Beeck EF. Incidence and costs of injuries in The Netherlands. Eur J Public Health 2006;16(3):272-8.

36. Meerding WJ, Bonneux L, Polder JJ, Koopmanschap MA, van der Maas PJ. Demographic and epidemiological determinants of healthcare costs in Netherlands: cost of illness study. BMJ 1998;317(7151):111-5.

37. Polder JJ, Takken J, W.J. M, Kommer GJ, Stokx LJ. Kosten van Ziekten in Nederland. De zorgeuro ontrafeld. Bilthoven: Rijksinstituut voor Volksgezondheid en Milieu, 2002: 1-304.

38. American Geriatrics Society BGS, and American Academy of Orthopaedic Surgeons Panel on Falls Prevention. Guideline for the prevention of falls in older persons. J Am Geriatr Soc 2001;49(5):664-72.

39. Scuffham $\mathrm{P}$, Chaplin S, Legood R. Incidence and costs of unintentional falls in older people in the United Kingdom. J Epidemiol Community Health 2003;57(9):740-4.

40. Carroll NV, Slattum PW, Cox FM. The cost of falls among the community-dwelling elderly. J Manag Care Pharm 2005;11(4):307-16.

41. Newton JL, Kyle P, Liversidge P, Robinson G, Wilton K, Reeve P. The costs of falls in the community to the North East Ambulance Service. Emerg Med J 2006;23(6):479-81.

42. Stevens JA, Corso PS, Finkelstein EA, Miller TR. The costs of fatal and non-fatal falls among older adults. Inj Prev 2006;12(5):290-5.

43. Roudsari BS, Ebel BE, Corso PS, Molinari NA, Koepsell TD. The acute medical care costs of fallrelated injuries among the U.S. older adults. Injury 2005;36(11):1316-22.

44. Polder JJ, Achterberg PW. Cost of illness in the Netherlands. Highlights. Bilthoven: National Institute for Public Health and the Environment, 2004: 1-19.

45. Stalenhoef PA, Diederiks JP, Knottnerus JA, Kester AD, Crebolder HF. A risk model for the prediction of recurrent falls in community-dwelling elderly: a prospective cohort study. J Clin Epidemiol 2002;55(11):1088-94. 
46. Stel VS, Pluijm SM, Deeg DJ, Smit JH, Bouter LM, Lips P. A classification tree for predicting recurrent falling in community-dwelling older persons. J Am Geriatr Soc 2003;51(10):1356-64.

47. Tromp AM, Pluijm SM, Smit JH, Deeg DJ, Bouter LM, Lips P. Fall-risk screening test: a prospective study on predictors for falls in community-dwelling elderly. J Clin Epidemio/ 2001;54(8):83744.

48. Chang JT, Ganz DA. Quality indicators for falls and mobility problems in vulnerable elders. J Am Geriatr Soc 2007;55 Suppl 2:S327-34.

49. Cesari M, Landi F, Torre S, Onder G, Lattanzio F, Bernabei R. Prevalence and risk factors for falls in an older community-dwelling population. J Gerontol A Biol Sci Med Sci 2002; 57(11):M722-6.

50. Stalenhoef $P$, Crebolder HF, Knottnerus A, F. Hvd. Incidence, risk factors and consequences of falls among elderly subject living in the community. Eur J Pub Health 1997;7:328-334.

51. Koch M, Gottschalk M, Baker DI, Palumbo S, Tinetti ME. An impairment and disability assessment and treatment protocol for community-living elderly persons. Phys Ther 1994;74(4):286-94.

52. Campbell AJ, Borrie MJ, Spears GF. Risk factors for falls in a community-based prospective study of people 70 years and older. J Gerontol 1989;44(4):M112-7.

53. Poulstrup A, Jeune B. Prevention of fall injuries requiring hospital treatment among community-dwelling elderly. Eur J Pub Health 2000;10:45-50.

54. Feder G, Cryer C, Donovan S, Carter Y. Guidelines for the prevention of falls in people over 65. The Guidelines' Development Group. BMJ 2000;321(7267):1007-11.

55. Graafmans WC, Ooms ME, Hofstee HM, Bezemer PD, Bouter LM, Lips P. Falls in the elderly: a prospective study of risk factors and risk profiles. Am J Epidemiol 1996;143(11):1129-36.

56. CBO KvdG. Richtlijn preventie van valincidenten bij ouderen. 2004.

57. Rizzo JA, Baker DI, McAvay G, Tinetti ME. The cost-effectiveness of a multifactorial targeted prevention program for falls among community elderly persons. Med Care 1996;34(9):954-69.

58. Smith RD, Widiatmoko D. The cost-effectiveness of home assessment and modification to reduce falls in the elderly. Aust N Z J Public Health 1998;22(4):436-40.

59. Salkeld G, Cumming RG, O'Neill E, Thomas M, Szonyi G, Westbury C. The cost effectiveness of a home hazard reduction program to reduce falls among older persons. Aust $N$ Z J Public Health 2000;24(3):265-71.

60. Robertson MC, Devlin N, Gardner MM, Campbell AJ. Effectiveness and economic evaluation of a nurse delivered home exercise programme to prevent falls. 1: Randomised controlled trial. BMJ 2001;322(7288):697-9.

61. Robertson MC, Devlin N, Scuffham P, Gardner MM, Buchner DM, Campbell AJ. Economic evaluation of a community based exercise programme to prevent falls. J Epidemiol Community Health 2001;55(8):600-6.

62. Robertson MC, Gardner MM, Devlin N, McGee R, Campbell AJ. Effectiveness and economic evaluation of a nurse delivered home exercise programme to prevent falls. 2: Controlled trial in multiple centres. BMJ 2001;322(7288):701-4.

63. Campbell AJ, Robertson MC, La Grow SJ, et al. Randomised controlled trial of prevention of falls in people aged $>$ or $=75$ with severe visual impairment: the VIP trial. BMJ 2005; 331(7520):817.

64. Beard J, Rowell D, Scott D, et al. Economic analysis of a community-based falls prevention program. Public Health 2006;120(8):742-51.

65. Goossens ME, Rutten van Molken MP, Vlaeyen JW, van der Linden SM. The cost diary: a method to measure direct and indirect costs in cost-effectiveness research. J Clin Epidemiol 2000;53(7):688-95. 
66. Drummond MF, Sculpher MJ, Thorrance GW, O'Brien BJ, Stoddart GL. Methods for the Economic Evaluation of Health Care Programmes. Third ed. New York: Oxford University Press, 2005.

67. Freer CB. Health diaries: a method of collecting health information. $J R$ Coll Gen Pract 1980;30(214):279-82.

68. Richardson A. The health diary: an examination of its use as a data collection method. $J$ Adv Nurs 1994;19(4):782-91.

69. van den Brink M, van den Hout WB, Stiggelbout AM, Putter H, van de Velde CJ, Kievit J. Selfreports of health-care utilization: diary or questionnaire? Int J Technol Assess Health Care 2005;21(3):298-304.

70. Lamoureux EL, Chou SL, Larizza MF, Keeffe JE. The reliability of data collection periods of personal costs associated with vision impairment. Ophthalmic Epidemiol 2006;13(2):121-6.

71. Tjalma-den Oudsten H, Bleijenberg C, Kaspers F, Boom N. Civil participation under the Social Support Act of the Netherlands. The Hague: SGBO, Research and Consultancy Bureau of the Association of Dutch Municipalities, 2006. 


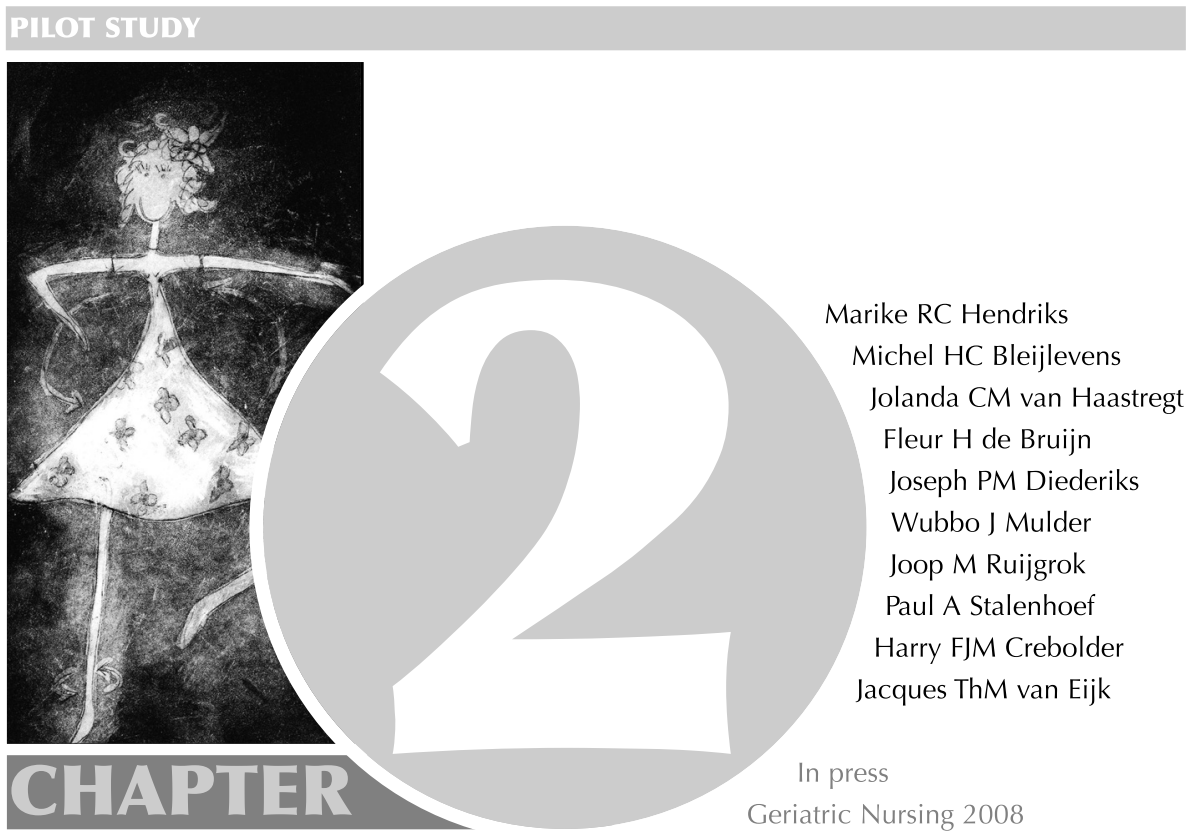




\begin{abstract}
Falling is a common problem among elderly people and has many negative consequences. In the Netherlands there is a need for effective fall prevention interventions aimed at elderly persons with an increased risk of falling. For this reason, we adapted a successful British fall prevention programme comprising a medical and occupational-therapy assessment to the Dutch healthcare setting. This paper describes the adaptation of this programme and a pilot study to assess its feasibility in Dutch healthcare according to the implementers of the intervention as well as the participants $(n=21)$.

This study showed that the Dutch intervention protocol is feasible in Dutch healthcare for both participants and implementers of the program. However, minor refinement of the intervention, is warranted to further improve its feasibility. The structured approach to adapt and pre-test an intervention protocol appeared to be essential when aiming to implement a complex intervention programme in a different healthcare setting.
\end{abstract}




\section{INTRODUCTION}

Falling is a common problem among elderly people. Falls are associated with an increase in morbidity and healthcare utilization, resulting in increased healthcare costs. ${ }^{1-6}$ Furthermore, people who have sustained a fall are at greater risk of falling again. ${ }^{7,8}$ In search of an effective intervention to prevent falls, many programmes have been developed and evaluated. Currently, there is considerable evidence for the effectiveness of multifaceted intervention programmes. ${ }^{7,9,10}$ One such multifaceted programme is a multidisciplinary programme developed by Close and colleagues (1999). ${ }^{8}$ This programme comprising a medical and occupational-therapy assessment has been evaluated in a randomized controlled trial (PROFET) and had favorable effects on falls and functioning. ${ }^{8}$ The PROFET study was aimed at people who had recently experienced an injurious fall. Persons who have recently experienced an injurious fall are at increased risk for additional falls, and may be considered a well motivated group for fall prevention. ${ }^{8}$ In the Netherlands there is a need for successful intervention programmes aimed at reducing falls among elderly persons who are at increased risk for falls. Therefore, we adjusted this successful British programme in order to develop a programme which fits in well with Dutch healthcare. The purpose of the present paper is to describe the adaptation of the intervention program, and subsequently to describe the feasibility of this adjusted programme according to implementers and participants.

\section{ADAPTATION OF THE INTERVENTION PROGRAM}

First, because no detailed intervention protocol was available of the British program, we had to reconstruct the intervention protocol based on the information provided in the literature ${ }^{8}$ and email communication with the main author, who was also the principal investigator of the British program. The PROFET study was targeted at elderly people, aged 65 years and over, who were living in the community in the London urban areas and attended the accident and emergency (A\&E) department with a primary diagnosis of a fall. A fall was defined as: inadvertently coming to rest on the ground or other lower level with or without loss of consciousness and other than as a consequence of sudden onset of paralysis, epileptic seizure, excess of alcohol intake, or overwhelming external force. ${ }^{8}$ Participants assigned to the intervention group 
underwent a medical and occupational-therapy assessment. The medical assessment in the British programme was performed by a geriatrician at a day hospital. The assessment comprised a comprehensive general examination and a detailed assessment of visual acuity, ${ }^{11}$ peripheral nervous system, ${ }^{12}$ balance, cognition, ${ }^{13}$ affect $^{14}$ and prescribed medications. On completion of the assessment, a primary cause for the index fall was assigned, and any identified risk factors for future falls were documented. When the geriatrician felt more detailed investigation or assessment was necessary, a referral was made to the relevant services. After the medical assessment, a single home visit was made by an occupational therapist for an assessment of functioning, ${ }^{15}$ potential environmental hazards and psychological consequences of the fall, ${ }^{16}$ followed by advice, education, and referrals if necessary. ${ }^{8}$ An overview of the contents of the medical and occupational-therapy assessment in the British programme can be found in of table 1 and table 2 respectively.

After reconstructing the program, we searched the literature for Dutch versions of the assessments used in the British protocol. If these were not available, we translated the assessments into Dutch or searched for comparable validated instruments. Next, we organized a meeting, structured according to the nominal group approach, ${ }^{17}$ to achieve consensus about possible protocol adaptations. Nine Dutch fall prevention experts from different disciplines (neurology, gerontology, geriatrics, geriatric nursing, physiotherapy, social and family medicine, occupational therapy and rehabilitation medicine) joined the meeting. These experts discussed the location, the disciplines of the implementers, and the content and assessment tools of the intervention program. The criterion of consensus was defined as $70 \%$ agreement on each topic discussed. In case of disagreement, we again searched the literature for relevant information about the disputed topic, discussed the topic with potential implementers of the intervention programme and other experts in fall prevention, and solved it.

The British programme was performed at a day hospital. Since day hospitals do not exist in the Netherlands, another suitable location had to be chosen. The consensus panel unanimously recommended that the medical assessment should take place in a well-equipped and patient-friendly environment, where participants can spend the waiting times between the assessments in a pleasant atmosphere and do not feel hospitalized. The panel recommended a department outside the actual University Hospital building, namely a recovery and rehabilitation clinic (after-care hospital). 
The British medical assessment was implemented by a geriatrician. To improve efficiency, the consensus panel unanimously recommended that the geriatrician could be assisted by a geriatric nurse doing assessments of cognition, affect, balance and mobility. Although the consensus panel recommended exact replication of most assessments, they proposed some additional assessments and recommended leaving out certain other assessments. An overview of the medical assessment of the Dutch intervention programme can be found in table 1. Reasons for adaptations of assessments are explained in table 3.

The panel underlined the role of the general practitioner (GP) as a gatekeeper in Dutch healthcare. In the Netherlands, (non-acute) referrals to medical specialists (secondary care) are implemented through the participants' GP (primary care). ${ }^{18,19}$ To improve efficiency and to increase the chances of successful implementation of the programme in regular care in the Netherlands in the future, the consensus panel recommended that on completion of the medical assessment, the geriatrician should evaluate the results and send a written summary of these results to the participant's GP. If necessary, this report should include recommendations for further referral to other relevant services. Participants should be recommended to contact their GP to be informed about the possible recommendations and/or referral resulting from the medical assessment. The GPs could then take action if they agreed with the recommendations and/or referrals to other services, as would have been the procedure in usual care. Furthermore, an intervention is most likely to be performed correctly if it fits in with daily routine and when those implementing it are familiar with the assessments. One way to achieve this is to train the implementers, another is to integrate the intervention as much as possible in regular healthcare. Whereas the British protocol described predefined cut-off points for assessments, the consensus panel recommended to avoid predefined cut-off points when no cut-off points were used in usual Dutch healthcare or no set of guidelines was available. Another reason for avoiding the use of cut-off points was that fall-related problems are multifactorial and overlapping; a person's clinical status is more than merely the sum of different diseases or impairments. ${ }^{20}$ Whether a particular impaired function results in an impairment or risk factor for falling, and should thus be addressed, depends on the participant's overall health status. Therefore, intervention decision rules cannot be prescribed beforehand, but should incorporate clinical judgment. 
After the medical assessment, as in the British program, an occupational therapist (OT) should perform a functional and environmental assessment at the participant's home. However, in contrast to the PROFET study, we chose to measure daily functioning by means of the 15-item Frenchay Activity Index (FAI) ${ }^{21}$ because the FAI shows no ceiling effect as the Barthel Index did in the PROFET study. ${ }^{8}$ The FAI has shown to be reliable, having a good construct validity, $^{22}$ and proved to be suitable for the general population of elderly people. $^{23}$ Moreover, the FAI focuses primarily on complex activities like housekeeping, recreation, hobbies and social interaction. These so-called instrumental abilities of daily living (IADL) may affect someone's quality of life considerably. ${ }^{21}$ Besides the FAI score, function should be assessed by means of the usual checklist of the local agency deciding on eligibility for care, aids and devices. ${ }^{24,25}$ Environmental hazards should be identified by means of a Dutch home-safety checklist. ${ }^{26}$ This checklist corresponds with that of the UK Health and Safety Executive, ${ }^{8}$ but is more extensive and in Dutch. On completion of the occupational-therapy assessment, recommendations should be made with regard to behavioural change, functional needs and safety within the home environment. Recommendations concerning technical aids and adaptations or additional support should be referred to social and community services. The panel recommended sending a personalized written description of the OT recommendations to the participants as well as their GPs, which may function as a reminder, and therefore might increase compliance. An overview of the occupational-therapy assessment of the Dutch intervention programme can be found in table 2 . 
Table 1 Overview of the medical assessments in the original British and adapted Dutch programmes*

\begin{tabular}{|c|c|c|c|}
\hline \multirow{2}{*}{\multicolumn{2}{|c|}{$\begin{array}{c}\text { Original British Programme - PROFET Study } \\
\text { Implementers }\end{array}$}} & \multirow{2}{*}{\multicolumn{2}{|c|}{$\frac{\text { Dutch Programme - Pilot Study }}{\text { Implementers }}$}} \\
\hline & & & \\
\hline \multicolumn{2}{|l|}{ Physician } & \multicolumn{2}{|l|}{$\begin{array}{l}\text { Geriatrician } \\
\text { Geriatric Nurse }\end{array}$} \\
\hline Assessments & Details/Tools/Test Batteries & Assessments & Details/Tools/Test Batteries \\
\hline $\begin{array}{l}\text { Standard examina- } \\
\text { tion }\end{array}$ & $\begin{array}{l}\text { Cardiovascular, respiratory, } \\
\text { abdominal, and neurologi- } \\
\text { cal system }\end{array}$ & $\begin{array}{l}\text { Standard examina- } \\
\text { tion }\end{array}$ & $\begin{array}{l}\text { Cardiovascular, respiratory, } \\
\text { and abdominal system }\end{array}$ \\
\hline Blood pressure & Supine and erect & Blood pressure & Supine and erect \\
\hline Vision & $\begin{array}{l}\text { Visual acuity (Snellen) } \\
\text { Visual fields } \\
\text { Cataract (Red Reflex) }\end{array}$ & Sense of hearing & $\begin{array}{l}\text { Visual acuity (Snellen) } \\
\text { Visual fields (confrontation) }^{11} \\
\text { Stereoscopic vision (TNO) } \\
\text { Whispered voice test }\end{array}$ \\
\hline $\begin{array}{l}\text { Peripheral nervous } \\
\text { system (focused on } \\
\text { legs) }\end{array}$ & $\begin{array}{l}\text { Tone \& Power (MRC } \\
\text { Scale) } \\
\text { Sensation (light touch) } \\
\text { Vibration (tuning fork first } \\
\text { metatarsophalangeal joint) } \\
\text { Proprioception (great toe) }\end{array}$ & $\begin{array}{l}\text { Peripheral nervous } \\
\text { system }\end{array}$ & $\begin{array}{l}\text { Tone \& Power (MRC Scale) } \\
\text { Sensation (monofilaments) } \\
\text { Vibration (tuning fork) } \\
\text { Proprioception (great toe) }\end{array}$ \\
\hline $\begin{array}{l}\text { Locomotor } \\
\text { apparatus: lumbar } \\
\text { spine, hips, knees } \\
\text { and feet }\end{array}$ & $\begin{array}{l}\text { Joint deformity } \\
\text { Range of movement }\end{array}$ & $\begin{array}{l}\text { Locomotor } \\
\text { apparatus: lumbar } \\
\text { spine, upper and } \\
\text { lower extremities }\end{array}$ & $\begin{array}{l}\text { Joint deformity } \\
\text { Range of movement } \\
\text { Handgrip dynamometry }\end{array}$ \\
\hline $\begin{array}{l}\text { Foot care and } \\
\text { footwear (subjective } \\
\text { assessment) }\end{array}$ & $\begin{array}{l}\text { Nail and skin care } \\
\text { Footwear }\end{array}$ & Feet and footwear & $\begin{array}{l}\text { Callus, skin ulcers, oedema } \\
\text { and arterial pulsations } \\
\text { Footwear }\end{array}$ \\
\hline Balance & $\begin{array}{l}\text { One leg test ( } 10 \text { seconds } \\
\text { with eyes open unable: } \\
\text { impaired) }\end{array}$ & Balance and mobili & $\begin{array}{l}\text { Romberg } \\
\text { Get Up and Go Test }\end{array}$ \\
\hline $\begin{array}{l}\text { Anthropometric } \\
\text { indices }\end{array}$ & $\begin{array}{l}\text { Triceps skin-fold thickness } \\
(\mathrm{mm}) \\
\text { Mid upper arm circumfer- } \\
\text { ence }(\mathrm{cm}) \\
\text { Body weight }(\mathrm{kg}) \\
\text { Height }(\mathrm{m})\end{array}$ & $\begin{array}{l}\text { Anthropometric } \\
\text { indices }\end{array}$ & $\begin{array}{l}\text { Body weight }(\mathrm{kg}) \\
\text { Height }(\mathrm{m})\end{array}$ \\
\hline Cognition & $\begin{array}{l}\text { Mini Mental State } \\
\text { Examination }{ }^{13}(\leq 24 \text { : } \\
\text { cognitive impairment })\end{array}$ & Cognition & $\begin{array}{l}\text { Mini Mental State Examina- } \\
\text { tion }^{13}\end{array}$ \\
\hline Affect & $\begin{array}{l}\text { Geriatric Depression } \\
\text { Scale }^{14}(\geq 6 \text { : possible } \\
\text { underlying depression })\end{array}$ & Affect & Geriatric Depression Scale ${ }^{14}$ \\
\hline Heart & $\begin{array}{l}\text { Electrocardiogram } \\
\text { Carotid Sinus Studies }\end{array}$ & Heart & Electrocardiogram \\
\hline Blood and urea & $\begin{array}{l}\text { Full blood count, urea, } \\
\text { electrolytes, liver function, } \\
\text { calcium, thyroid tests }\end{array}$ & Blood tests & If indicated \\
\hline Medication & & Medication & \\
\hline Referral And/ & Or Recommendation & Referral Anc & Or Recommendation \\
\hline $\begin{array}{l}\text { Direct referral to rel } \\
\text { of specialty within th } \\
\text { tion was proposed t }\end{array}$ & $\begin{array}{l}\text { evant service or department } \\
\text { ne hospital. Drug modifica- } \\
\text { o the GP, if necessary. }\end{array}$ & $\begin{array}{l}\text { No direct referral w } \\
\text { recommendations } 1 \\
\text { tion are sent to the } \\
\text { practitioner / family }\end{array}$ & $\begin{array}{l}\text { hin the hospital, but } \\
\text { esulting from this examina- } \\
\text { articipant's general } \\
\text { ohysician }\end{array}$ \\
\hline
\end{tabular}

* Differences between the original and Dutch programmes resulting from the process of adaptation are shown in italics. 
Table 2 Overview of occupational-therapy assessment in the original British and adapted Dutch programmes*

\begin{tabular}{|c|c|c|c|}
\hline \multicolumn{2}{|c|}{ Original British Programme - PROFET Study } & \multicolumn{2}{|c|}{ Dutch Program- Pilot Study } \\
\hline \multicolumn{2}{|c|}{ Implementer } & \multicolumn{2}{|c|}{ Implementers } \\
\hline \multicolumn{2}{|l|}{ Occupational Therapist } & \multicolumn{2}{|l|}{ Occupational Therapist } \\
\hline Assessments & Test Battery & Assessments & Test Battery \\
\hline \multirow[t]{2}{*}{ Function } & Barthel Index ${ }^{15}$ & Function 1 & $\begin{array}{l}\text { Checklist of the local } \\
\text { agency deciding on } \\
\text { eligibility for care, aids } \\
\text { and devices }{ }^{24,25}\end{array}$ \\
\hline & & Function 2 & $\begin{array}{l}\text { Frenchay Activity } \\
\text { Index }\end{array}$ \\
\hline Environmental hazards & $\begin{array}{l}\text { Checklist UK Health } \\
\text { and Safety Executive }\end{array}$ & Environmental hazards & $\begin{array}{l}\text { Dutch version of the } \\
\text { Home Checklist P.A. } \\
\text { Stalenhoef, } 1998^{26}\end{array}$ \\
\hline $\begin{array}{l}\text { Psychological conse- } \\
\text { quences of the fall }\end{array}$ & $\begin{array}{l}\text { Falls Handicap } \\
\text { Inventory }^{16}\end{array}$ & $\begin{array}{l}\text { Psychological conse- } \\
\text { quences of the fall }\end{array}$ & $\begin{array}{l}\text { Falls Handicap } \\
\text { Inventory }^{16}\end{array}$ \\
\hline \multicolumn{2}{|l|}{ Referral } & \multicolumn{2}{|l|}{ Referral } \\
\hline \multicolumn{2}{|c|}{$\begin{array}{l}\text { Advice and education about functional need } \\
\text { and safety within the home; minor home } \\
\text { adaptations and minor equipment were } \\
\text { supplied directly; participants who needed } \\
\text { technical aids, home adaptations, or additional } \\
\text { support were referred to social or hospital } \\
\text { services as usual. }\end{array}$} & \multicolumn{2}{|c|}{$\begin{array}{l}\text { Recommendations regarding adaptations to the } \\
\text { home environment, assistive devices, and home } \\
\text { care are referred to and delivered by social and } \\
\text { community services. A copy of the recommenda- } \\
\text { tions including recommendations of behavioural } \\
\text { change is sent to the participants as a reminder. }\end{array}$} \\
\hline
\end{tabular}

* Differences between the original and Dutch programmes resulting from the process of adaptation are shown in italics. 
Table 3 Overview of reasons for adaptations of assessments from the original British to the Dutch program

\begin{tabular}{|c|c|c|}
\hline Assessment & $\begin{array}{l}\text { Added / } \\
\text { rejected }\end{array}$ & Reason \\
\hline Stereoscopic vision $^{27}$ & Added & $\begin{array}{l}\text { A visual impairment is seldom merely caused by poor } \\
\text { visual acuity: depth perception also plays an important } \\
\text { role }^{30-32} \\
\text { Stereoscopic vision tests are simple and not time- } \\
\text { consuming }\end{array}$ \\
\hline Whispered voice test ${ }^{33}$ & Added & $\begin{array}{l}\text { Impaired sensory input (i.e. sense of hearing) is a risk } \\
\text { factor for imbalance and therefore probably for falls as } \\
\text { well }^{34}\end{array}$ \\
\hline Handgrip dynamometry test & Added & $\begin{array}{l}\text { Handgrip strength in the dominant hand is related to } \\
\text { total body muscle strength, and total body strength is a } \\
\text { good predictor of recurrent falls } s^{4,35}\end{array}$ \\
\hline Foot care & Rejected & Foot care assessment was regarded as too subjective \\
\hline Feet & Added & $\begin{array}{l}\text { Feet should be inspected for callus, skin ulcers, } \\
\text { oedema and arterial pulsations }\end{array}$ \\
\hline One-leg test & Rejected & $\begin{array}{l}\text { One-leg test is too difficult for elderly people in } \\
\text { general. } \\
\text { Difficulties in performing one-leg test may also be } \\
\text { caused by muscular problems }{ }^{36}, \text { such as decreased } \\
\text { strength of the hip extensors and flexors }\end{array}$ \\
\hline Romberg test ${ }^{28}$ & Added & $\begin{array}{l}\text { To assess the sense of position the Romberg test }{ }^{28} \text { was } \\
\text { preferred by and commonly used by examiners at the } \\
\text { Geriatrics Department }\end{array}$ \\
\hline Get Up and Go Test ${ }^{29}$ (GUGT) & Added & $\begin{array}{l}\text { The GUGT is a reliable and validated instrument to test } \\
\text { balance and gait } \\
\text { The GUGT was commonly used by examiners at the } \\
\text { Geriatrics Department }\end{array}$ \\
\hline $\begin{array}{l}\text { Triceps skin-fold thickness \& } \\
\text { mid upper arm circumference } \\
\text { (TST \& MUAC) }\end{array}$ & Rejected & $\begin{array}{l}\text { Assessment TST \& MUAC was regarded as too detailed } \\
\text { and time-consuming and therefore not expected to be } \\
\text { cost-effective } \\
\text { Height and weight provide sufficient anthropometric } \\
\text { information to be used as an indicator of the risk of } \\
\text { falls }\end{array}$ \\
\hline $\begin{array}{l}\text { Carotid Sinus Studies: carotid } \\
\text { sinus massage (CSM) and } \\
\text { auscultation }\end{array}$ & Rejected & $\begin{array}{l}\text { CSM as a diagnostic tool for the carotid hyperactivity } \\
\text { syndrome is not undisputed }{ }^{37} \text {, mainly because of its } \\
\text { rather drastic therapeutic implications (cardiac pacing), } \\
\text { and auscultation (as an assessment tool for carotid } \\
\text { atherosclerosis) is an imperfect screening test }{ }^{29} \\
\text { The results of Close's study are unclear with regard to } \\
\text { this particular part of the protocol }\end{array}$ \\
\hline $\begin{array}{l}\text { Full blood count, urea and } \\
\text { electrolytes, liver function } \\
\text { tests, calcium and thyroid } \\
\text { function tests and/or vitamin } \\
\text { B12 and folic acid }\end{array}$ & Rejected & $\begin{array}{l}\text { Only carried out if the geriatrician considered them } \\
\text { necessary }\end{array}$ \\
\hline
\end{tabular}




\section{METHODS OF THE PILOT STUDY}

\section{Design and participants}

Recruitment of participants for the pilot study took place during six weeks. Eligible participants were community-dwelling elderly people aged 65 years and over who attended the A\&E department of the University Hospital Maastricht for the consequences of a fall. Since the out of hours accident and emergency care in the town of Maastricht is carried out by the GP Cooperative (General Practitioner Cooperative), ${ }^{38,39}$ participants were selected at the GP Cooperative as well. People were excluded if they were unable to speak Dutch, cognitively impaired in combination with having no regular caregiver, admitted to a hospital or another institution (or planning to move house) during the intervention period, permanently wheelchair-dependent or bedridden and/or did not give informed consent. For practical reasons only those (eligible) persons who lived in the city of Maastricht were allocated to the intervention group, and underwent medical and occupational-therapy assessments, followed by recommendations or further referral if indicated. Persons living in surrounding areas received no intervention. The intervention period lasted one month, and participants were followed for one month after their inclusion. The study design and protocols of the pilot study were approved by the Medical Ethics Committee of the University Hospital and Maastricht University.

\section{Measurements and analyses}

We assessed the feasibility of the intervention program, by assessing the following topics: reasons for not joining the program; the extent to which the intervention protocol was applied as intended; feasibility of the assessments, recommendations and/or referral resulting from the assessments; perceived benefit and satisfaction among the participants of the intervention group and satisfaction of the medical and paramedical practitioners. Data were collected by means of structured registration forms, a self-administered questionnaire from the participants of the intervention group, and interviews with those who implemented the intervention programme after the intervention period. Analyses were done by means of descriptive statistics. 


\section{RESULTS}

\section{Participants}

Figure 1 shows the flow of participants during the pilot study. A total of 195 potentially eligible persons (55 men and 140 women) attended the A\&E Department and the GP Cooperative at the University Hospital of Maastricht with the consequences of a fall. Informed consent had been obtained from 36 persons to participate in the pilot study. The main reasons for not joining the study were 'not interested to participate' ( $n=57$ ), 'not living independently' (n $=34$ ) and 'unknown address' $(n=20)$. During the recruitment, we noticed that some persons tended to overestimate the contribution of external factors to their fall, and tended to underestimate the contribution of internal factors. The study population consisted of 12 men and 24 women with a mean age of 76, and 14 persons were living alone. In 20 persons the index fall had resulted in a fracture. Of the 36 eligible persons 21 were living in Maastricht and therefore allocated to the intervention group. Two persons withdrew before the medical assessment took place. Process evaluation questionnaires about the intervention programme were completed by 16 persons.

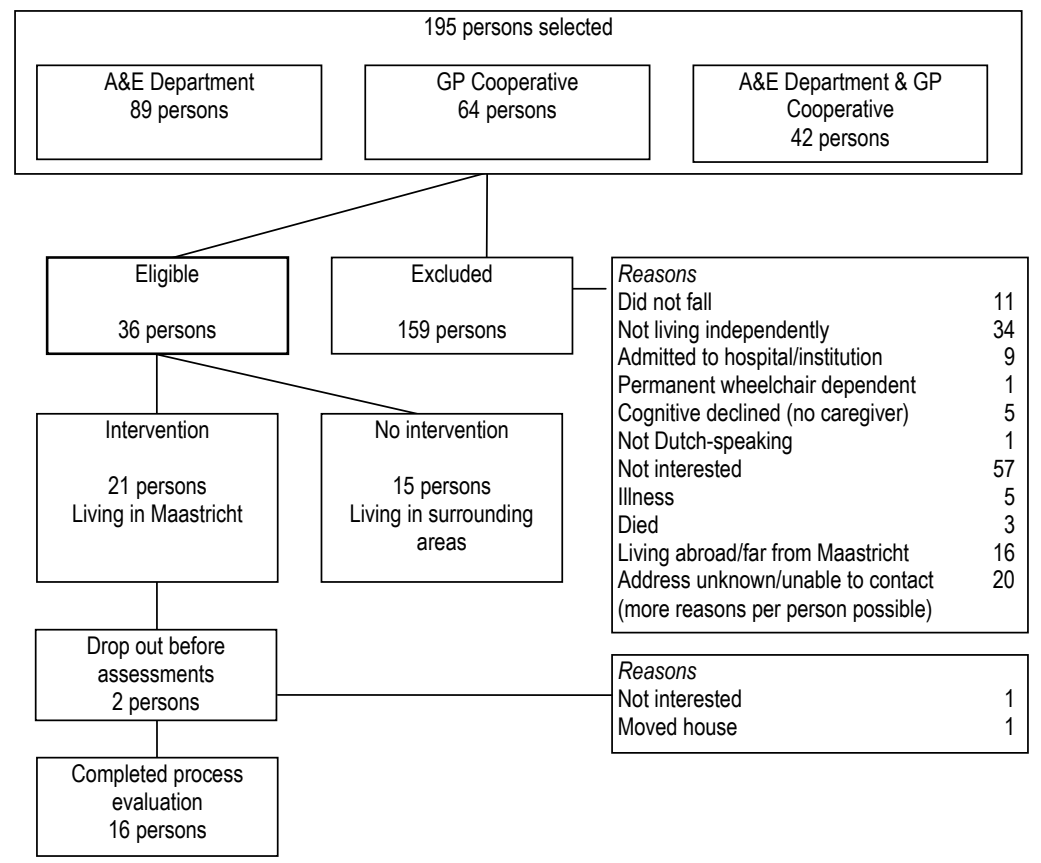

Figure 1 Participant flow of the pilot study 


\section{Results of the medical assessment}

Because two persons withdrew from the study before the medical assessment took place, 19 participants underwent the medical assessment. According to the geriatrician, the medical assessment was feasible and largely performed according to protocol, except that most participants were not able to recall their prescribed medications. To overcome this recall problem, the geriatrician recommended asking participants to bring their medications or a list of prescribed medications in future assessments. All assessments were filled on the registration forms. Furthermore, the geriatrician, considered himself to be insufficiently specialized to examine feet and footwear in detail and recommended to involve for example a rehabilitation physician (physiatrist).

The duration of the medical assessment varied between 45 to 60 minutes. Questionnaires revealed that most participants ( $n=15$ out of 16) perceived the duration of the medical assessment as acceptable. Only 3 participants found it difficult to perform the tasks and assessments, while 13 regarded them as simple. Most participants $(n=13)$ perceived the medical part of the intervention as beneficial.

The geriatrician perceived that most participants of the pilot study were in reasonably good physical condition. In 9 of the 19 participants the medical assessment resulted in recommendations and/or referral (details see table 4).

Table 4 Recommendations and/or referrals resulting from the medical assessment in the pilot study

\begin{tabular}{lc}
\hline Kind of recommendation/referral & Number of participants \\
\hline Dexa scan & 5 \\
Referral to cardiologist & 3 \\
Change prescribed medication & 3 \\
Blood tests & 1 \\
Referral to neurologist & 1 \\
Bandaging the legs & 1 \\
Total of recommendations/referrals & $9^{*}$ \\
\hline
\end{tabular}

* Participants can have more than one recommendation and/or referral.

Results of the medical assessment including the possible recommendations and/or referrals were sent to the participant's GP. Questionnaires revealed that half of the participants in the intervention group had not yet consulted their GP to get informed about the results of the medical assessment at the moment of the process evaluation (about 1-2 weeks after receiving the 
reminder to call their GP). All participants who asked their GP and received information or feedback about the assessment were satisfied about that.

The location chosen for the medical assessment (a recovery and rehabilitation clinic) was considered to be insufficiently equipped and it was difficult to reach by means of public transport. No ECG equipment was available, so this had to be brought in by the research team. Furthermore, if blood tests were necessary, participants had to go to the hospital by themselves afterwards.

Only 4 participants were able to visit the intervention location using their own means of transport. The other participants were picked up by taxi.

\section{Results of the occupational-therapy assessment}

After the medical assessment all participants were scheduled for a home visit by the occupational therapist. Because of scheduling problems, one participant did not receive the occupational-therapy assessment. Registration forms showed that the occupational-therapy assessment was performed according to protocol. The duration of the occupational-therapy home visit was about one hour. This was acceptable according to the majority of the participants ( $\mathrm{n}$ $=14$ out of 15). All participants found the occupational-therapy assessment beneficial and pleasant.

The occupational-therapy assessment resulted in at least one recommendation or suggestion for behavioural change for every participant. Furthermore, the majority of the participants $(n=11)$ received another recommendation for a home adaptation or assistive device (see table 5). Most participants $(n=12)$ indicated that they would definitely comply with the recommendations, and all participants found the recommendations useful. 
Table 5 Recommendations resulting from the occupational-therapy assessment in the pilot study

\begin{tabular}{|c|c|}
\hline Kind of recommendation & Number of participants \\
\hline Total home adaptations & $10^{*}$ \\
\hline Handrails or stair rails & 10 \\
\hline Leveling floors & 3 \\
\hline Lowering steps & 1 \\
\hline Installing automatic doors & 1 \\
\hline Total assistive devices & $3^{*}$ \\
\hline Walking devices (like rollators) & 3 \\
\hline Orthopedic footwear & 1 \\
\hline Total behavioural change & $18^{*}$ \\
\hline Removing loose carpets & 13 \\
\hline Use assistive devices & 13 \\
\hline Taking one's time & 8 \\
\hline Total of recommendations & 31 \\
\hline
\end{tabular}

* Participants can have more than one recommendation.

The occupational therapist judged the protocol to be feasible. Furthermore, the therapist also perceived most participants of the pilot study as being in reasonably good physical condition. The occupational therapist as well as the participants perceived the occupational therapist to be the right implementer to perform the occupational-therapy assessment.

\section{DISCUSSION}

This study showed that the Dutch intervention protocol turned out to be feasible in the Dutch situation for both participants and implementers of the program. Both medical and occupational-therapy assessments were applied as intended. Based on their experiences during the pilot the implementers made some recommendations to further optimize the intervention. Participants were satisfied with the intervention program, but half of them had not yet consulted their GP to get informed about the results of the medical assessment. According to the geriatrician, this may be explained by the short time between intervention and evaluation.

A limitation of the present study is that the interviews with medical and paramedical practitioners were performed by the investigators, and may therefore be susceptible to social desirable answers. To minimize social desirable answers from the participants we used self-administered questionnaires in stead of face to face interviews to assess the opinion of participants. 
This study showed the importance of a structured approach to adapting and pre-testing an intervention protocol when the aim is to implement it in a different healthcare setting. In order to fit the intervention in with Dutch regular care, we had to make a number of adaptations to the original protocol. Finally, the pilot study revealed that further optimizing of the adapted intervention was needed.

Based on the results of this feasibility study we recommend the following adaptations to optimize the intervention protocol. Because the location of the medical assessment during the pilot study was not adequately equipped and not accessible by public transport, we recommend performing the medical assessment in a hospital. Furthermore, because the geriatrician judged he was not specialized to examine feet and footwear in detail, and no recommendations about this aspect were given to the participants, we assessed whether an adjusted approach was desirable. We searched the literature, consulted a rehabilitation physician and other experts on fall prevention about standardized feet and footwear assessments, and recommend to add two assessments: a reliable footwear assessment form ${ }^{40}$ and a test with monofilaments to improve objective assessment of proprioception. Besides, we recommend involving a rehabilitation physician to perform these detailed and standardized assessments of feet and footwear.

With regard to future research, the pilot revealed that during the recruitment period some people overestimated the contribution of external factors to their fall. For example, people who were over walked by a dog, were excluded for the reason 'fall as a consequence of overwhelming external force' although it was a very small dog. In order to avoid the unjustified exclusion of eligible participants in future research, we recommend to broaden our definition of a fall: 'A fall is an event which results in a person coming to rest inadvertently on the ground or other lower level' ${ }^{41}$

Although the British programme showed promising effects in terms of reducing falls and functional decline in the British setting, this is no guarantee for its effectiveness in a different healthcare setting. This is especially true when an intervention is transposed to a different healthcare setting. Therefore, based on the results of the feasibility study presented in this paper a two group randomized controlled trial (ISRCTN 64716113; $\mathrm{n}=333$ ) $^{39}$ is currently being performed. This trial aims to assess the effectiveness and costeffectiveness of the final version of the programme in the Dutch healthcare setting. The results of this trial will be published as soon as they become available. 


\section{REFERENCES}

1. Speechley M, Tinetti M. Falls and injuries in frail and vigorous community elderly persons. J Am Geriatr Soc 1991;39(1):46-52.

2. Sattin RW, Rodriguez JG, DeVito CA, Wingo PA. Home environmental hazards and the risk of fall injury events among community-dwelling older persons. J Am Geriatr Soc 1998;46:669-76.

3. Nevitt MC, Cummings SR, Hudes ES. Risk factors for injurious falls: a prospective study. J Gerontol 1991;46(5):M164-70.

4. Blake AJ, Morgan K, Bendall MJ, et al. Falls by elderly people at home: prevalence and associated factors. Age Ageing 1988;17(6):365-72.

5. Tinetti ME, Speechley M, Ginter SF. Risk factors for falls among elderly persons living in the community. N Engl J Med 1988;319(26):1701-7.

6. Rizzo JA, Friedkin R, Williams CS, Nabors J, Acampora D, Tinetti ME. Health care utilization and costs in a Medicare population by fall status. Med Care 1998;36(8):1174-88.

7. Gillespie LD, Gillespie WJ, Robertson MC, Lamb SE, Cumming RG, Rowe BH. Interventions for preventing falls in elderly people. Cochrane Database Syst Rev 2003(4):CD000340.

8. Close J, Ellis M, Hooper R, Glucksman E, Jackson S, Swift C. Prevention of falls in the elderly trial (PROFET): a randomised controlled trial. Lancet 1999;353(9147):93-97.

9. Chang JT, Morton SC, Rubenstein LZ, et al. Interventions for the prevention of falls in older adults: systematic review and meta-analysis of randomised clinical trials. BMJ 2004; 328(7441):680.

10. Kannus P, Sievanen H, Palvanen M, Jarvinen T, Parkkari J. Prevention of falls and consequent injuries in elderly people. Lancet 2005;366(9500):1885-93.

11. Hetherington R. The Snellen chart as a test of visual acuity. Psychol Forsch 1954;24(4):349-57.

12. van der Ploeg RJ, Oosterhuis HJ. Fysische diagnostiek--het meten van spierkracht. [Physical examination--measurement of muscle strength]. Ned Tijdschr Geneeskd 2001;145(1):19-23.

13. Folstein MF, Folstein SE, McHugh PR. "Mini-mental state". A practical method for grading the cognitive state of patients for the clinician. J Psychiatr Res 1975;12(3):189-98.

14. Brink TL, Yesavage JA, Lum O, Heersema PH, Adey M, Rose TL. Screening tests for geriatric depression. Clin gerontologist 1982;1(1):37-43.

15. Mahoney FI, Barthel DW. Functional Evaluation: The Barthel Index. Md State Med J 1965;14:61-5.

16. Rai GS, Kiniorns M, Wientjes H. Falls Handicap Inventory (FHI)--an instrument to measure handicaps associated with repeated falls. J Am Geriatr Soc 1995;43(6):723-4.

17. Van de Ven AH, Delbecq AL. The nominal group as a research instrument for exploratory health studies. Am J Public Health 1972;62(3):337-42.

18. Meyboom de Jong B, Smith RJ, Hiddema van der Wal A, van der Werf GT. De poortwachtersfunctie van de huisarts onderzocht met verwijzingen en verwijskaarten. [ The family physician's sentinel function studied with reference to referrals and referral cards]. Ned Tijdschr Geneeskd 1996;140(29):1499-504.

19. Kulu Glasgow I, Delnoij D, de Bakker D. Self-referral in a gatekeeping system: patients' reasons for skipping the general-practitioner. Health Policy 1998;45(3):221-38.

20. Tinetti ME. Performance-oriented assessment of mobility problems in elderly patients. J Am Geriatr Soc 1986;34(2):119-26.

21. Schuling J, de Haan R, Limburg M, Groenier KH. The Frenchay Activities Index. Assessment of functional status in stroke patients. Stroke 1993;24(8):1173-7. 
22. Turnbull JC, Kersten P, Habib M, McLellan L, Mullee MA, George S. Validation of the Frenchay Activities Index in a general population aged 16 years and older. Arch Phys Med Rehabil 2000; 81(8):1034-8.

23. Van Haastregt JC, Diederiks JP, van Rossum E, de Witte LP, Voorhoeve PM, Crebolder HF. Effects of a programme of multifactorial home visits on falls and mobility impairments in elderly people at risk: randomised controlled trial. BMJ 2000;321(7267):994-8.

24. Rooken M, Van der Ven S. Standaard voor het afleggen van een huisbezoek door ergotherapeuten: Stroomschema en formulier. NVE-standaard. Utrecht: Nederlandse Vereniging voor Ergotherapie, 2001.

25. Ummels C, Lahaye V. Ergotherapeutische registratielijst voor een ADL-zelfverzorgingsobservatie: Handleiding en formulier. NVE-standaard. Utrecht: Nederlandse Vereniging voor Ergotherapie, 2001.

26. Stalenhoef P, Diederiks J, Knottnerus A, de Witte L, Crebolder H. How predictive is a homesafety checklist for indoor fall risk in the elderly living in the community? Eur J Gen Pract 1998; 4:114-120.

27. Walraven J. Amblyopia screening with random-dot stereograms. Am J Ophthalmol 1975; 80(5):893-900.

28. Van Vaerenbergh J, Broos P. Positieve Romberg-test en het voorspellen van vallen bij bejaarden. [Positive Romberg test and the probability of falls in the aged]. Tijdschr Gerontol Geriatr 1990;21(2):71-4.

29. Mathias S, Nayak US, Isaacs B. Balance in elderly patients: the "get-up and go" test. Arch Phys Med Rehabil 1986;67(6):387-9.

30. Nevitt MC, Cummings, S.R., Kidd, S., Black, D. Risk factor for recurrent nonsyncopal falls. JAMA 1989; 261:2663-2668.

31. Harwood RH. Visual problems and falls. Age Ageing 2001;30 Suppl 4:13-8.

32. Abdelhafiz $\mathrm{AH}$, Austin $\mathrm{CA}$. Visual factors should be assessed in older people presenting with falls or hip fracture. Age Ageing 2003;32(1):26-30.

33. Eekhof JA, de Bock GH, de Laat JA, Dap R, Schaapveld K, Springer MP. The whispered voice: the best test for screening for hearing impairment in general practice? $\mathrm{Br} J$ Gen Pract 1996; 46(409):473-4.

34. Gerson LW, Jarjoura D, McCord G. Risk of imbalance in elderly people with impaired hearing or vision. Age Ageing 1989;18(1):31-4.

35. Stalenhoef PA, Diederiks JP, Knottnerus JA, Kester AD, Crebolder HF. A risk model for the prediction of recurrent falls in community-dwelling elderly: a prospective cohort study. J Clin Epidemiol 2002;55(11):1088-94.

36. Era P, Heikkinen E. Postural sway during standing and unexpected disturbance of balance in random samples of men of different ages. J Gerontol 1985;40(3):287-95.

37. McAnulty JH. Carotid sinus massage in patients who fall: will it define the role of pacing? J Am Coll Cardiol 2001;38(5):1497.

38. Van Uden CJ, Winkens RA, Wesseling GJ, Crebolder HF, van Schayck CP. Use of out of hours services: a comparison between two organisations. Emerg Med J 2003;20(2):184-7.

39. Hendriks MR, van Haastregt JC, Diederiks JP, Evers SM, Crebolder HF, van Eijk JT. Effectiveness and cost-effectiveness of a multidisciplinary intervention programme to prevent new falls and functional decline among elderly persons at risk: design of a replicated randomised controlled trial [ISRCTN64716113]. BMC Public Health 2005;5(1):6.

40. Menz HB, Sherrington C. The Footwear Assessment Form: a reliable clinical tool to assess footwear characteristics of relevance to postural stability in older adults. Clin Rehabil 2000; 14(6):657-64.

41. The prevention of falls in later life. Dan Med Bull 1987;34 Suppl 4:1-24. 

EFFECTIVENESS AND COST-EFFECTIVENESS OF A MULTIDISCIPLINARY INTERVENTION PROGRAMME TO PREVENT NEW FALLS AND FUNCTIONAL DECLINE AMONG ELDERLY PERSONS AT RISK: DESIGN OF A REPLICATED RANDOMISED CONTROLLED TRIAL [ISRCTN64716113]

\section{DESICN}

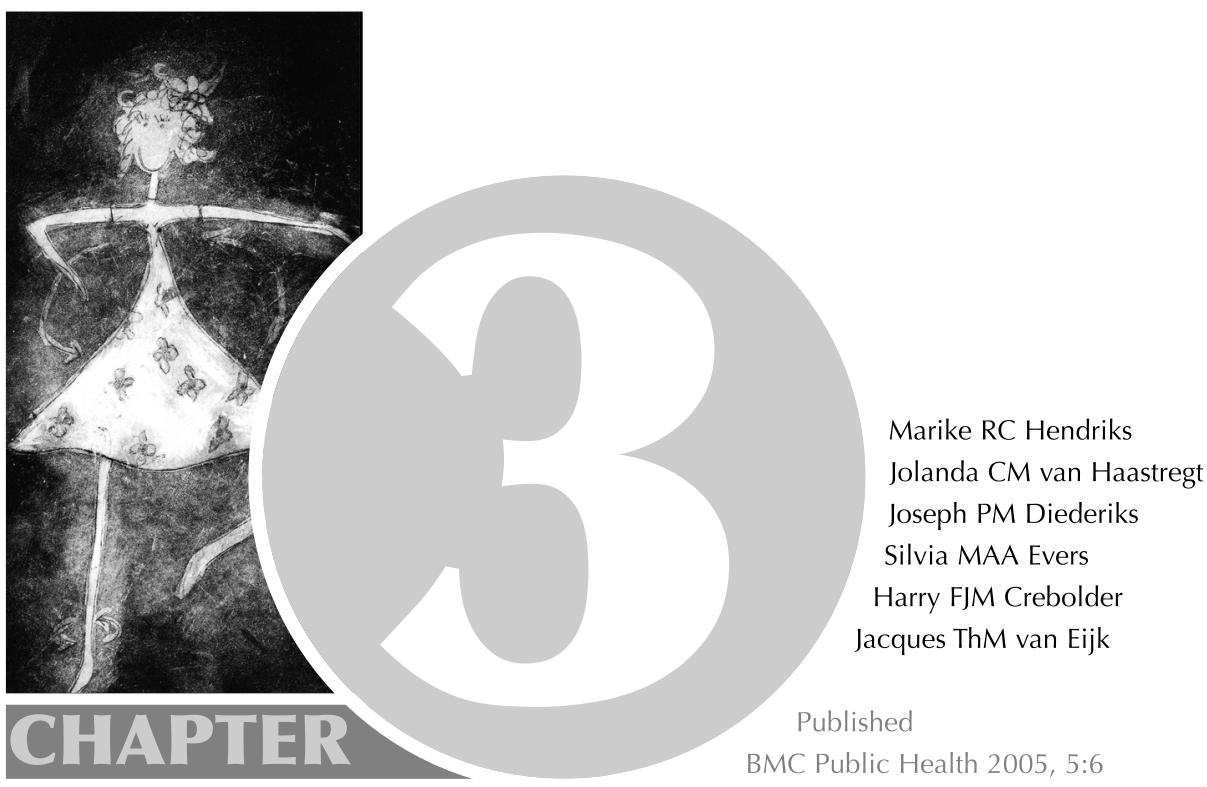




\section{ABSTRACT}

Background Falls are common among community-dwelling elderly people and can have a considerable impact on quality of life and healthcare costs. People who have sustained a fall are at greater risk of falling again.

We replicated a British randomized controlled trial which demonstrated the effectiveness of a multidisciplinary intervention programme to prevent falls.

The objective is to describe the design of a replication study evaluating a multidisciplinary intervention programme on recurrent falls and functional decline among elderly persons at risk. The study consists of an effect evaluation, an economic evaluation and a process evaluation.

Methods/design The programme is aimed at community-dwelling elderly people aged 65 years or over who have visited an accident and emergency department (A\&E department) or a general practitioners' cooperative (GP cooperative) because of a fall.

The design involves a two-group randomized controlled trial. Participants are followed for twelve months after baseline. The intervention programme consists of a detailed medical and occupational-therapy assessment with referral to relevant services if indicated. People in the control group receive usual care.

The main outcome measures of the effect evaluation are number of falls and daily functioning. The economic evaluation will be performed from a societal perspective. A process evaluation will be carried out to evaluate the feasibility of the intervention programme. 


\section{BACKGROUND}

\section{Publishing the design of a study}

This article describes the design of a replication of a randomized controlled trial (RCT) evaluating the effectiveness and cost-effectiveness of a multidisciplinary intervention programme to prevent further falls among elderly people at risk.

Publishing the design and protocol of a study before results are available is important for several reasons. A published protocol allows easier comparison between what was originally intended and hypothesised and what was actually done, ${ }^{1}$ and it gives readers greater insight into the methodological quality of a study. Furthermore, it has often been recognised that negative or adverse outcomes are less likely to be published..$^{1-3}$ Publishing the design of a study before its start announces that a study will be undertaken, which encourages publication of the results and in any case informs researchers where they can find the data for inclusion in systematic reviews. ${ }^{1,2}$ Thus, publishing a design article can prevent publication bias.

\section{Prevention of falls}

About one-third of people over the age of 65 fall at least once a year. ${ }^{4}$ People who have fallen show an increase in morbidity, mortality and healthcare utilization, ${ }^{5}$ which implies increased healthcare costs. In addition, people who have sustained a fall are at greater risk of falling again. ${ }^{5}$ Since preventing falls has been a matter of interest for years, many programmes aimed at preventing falls have been developed and evaluated. Unfortunately, many of these have turned out to be ineffective. ${ }^{4}$ However, there is now considerable evidence of the effectiveness of multifaceted interventions. Programmes likely to be effective in preventing falls among elderly people are multidisciplinary, multifactorial programmes screening for health and environmental risk factors and offering interventions, both for the general population of community-dwelling elderly people and for elderly people with a history of falling selected because of known risk factors. ${ }^{4}$ An example of such an intervention programme is the successful programme developed by Close et al.. ${ }^{5}$ This programme is aimed at people aged 65 years or older who live in the community and have visited an accident and emergency department because of a fall. 
The intervention programme consists of a detailed medical and occupationaltherapy assessment with referral to relevant services if indicated. The intervention has been evaluated in a randomized controlled trial, which demonstrated that this multidisciplinary intervention implemented among people at risk was highly effective in reducing the number of recurrent falls and associated injuries in London (United Kingdom). ${ }^{5}$

Because details of the status of the participants, the context of the intervention and the content and presentation appear to be critical, it has been recommended to re-evaluate effective intervention programmes in different healthcare systems. ${ }^{4}$ We therefore decided to evaluate the effectiveness of the intervention developed by Close $^{5}$ in Dutch healthcare, by replicating this study in the Netherlands.

\section{Objective and research questions}

The main objective of our current study is to evaluate the effects of a multidisciplinary intervention programme on recurrent falls and functional decline among elderly persons who have visit a general practitioners' cooperative (GP cooperative) and/or an accident and emergency department (A\&E department) because of a fall. This objective has resulted in the following research questions.

1. Is a multidisciplinary intervention programme more effective than usual care in preventing new falls and functional decline among communitydwelling elderly people who visit a GP cooperative and/or A\&E department at a hospital because of a fall?

2. Is the multidisciplinary intervention programme cost-effective compared to usual care when assessed from a societal perspective?

Besides the effect evaluation and economic evaluation, a process evaluation is being carried out to assess the feasibility and applicability of the intervention programme for those receiving and implementing the intervention.

\section{DESIGN AND METHODS}

\section{Design}

Figure 1 shows the design of the study presented, which is a two-group randomized controlled trial. At this stage, the randomization process has already been completed. Randomization was achieved by means of computer- 
ised alternative allocation and performed by an external agency. Randomization took place after completion of a self-administered baseline questionnaire. People allocated to the control group received usual healthcare, while people in the intervention group underwent a medical and occupationaltherapy assessment. The intervention period is scheduled to last for a maximum of 3.5 months after the baseline measurement. After baseline measurement, all subjects are followed for a twelve-month period. During this followup period, falls and healthcare utilization are measured continuously. Subjects are contacted monthly by telephone for an interview about their falls and healthcare utilization. In addition, self-administered questionnaires are sent to the subjects after four and twelve months.

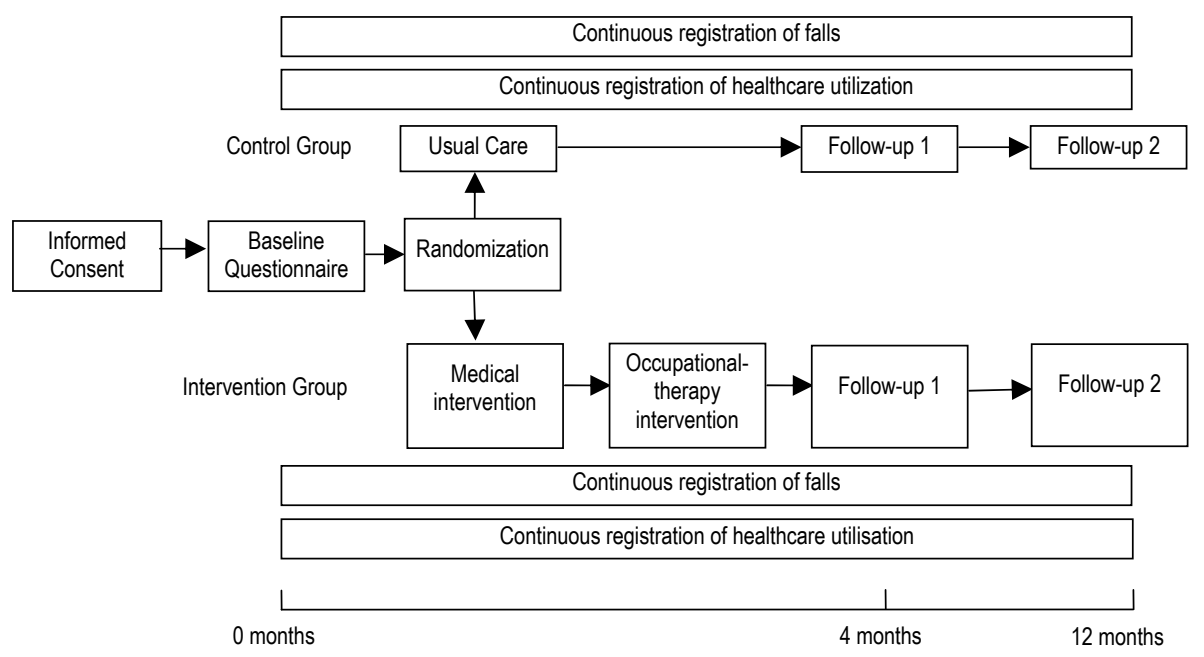

Figure 1 Study Design

We have taken various measures to ensure blinding in the data collection process. Questionnaires are collected anonymously and sorted by number. Follow-up measurements by phone are contracted out to an independent call centre, whose operators are unaware whether the subjects have been allocated to the intervention or the control group. The study design and protocols were approved by the Medical Ethics Committee of the University Hospital and University of Maastricht. 


\section{Target population}

Various studies have been conducted to assess the effectiveness of programmes to prevent falls. Although most studies were aimed at the general population of elderly people, details of the status of the participants appear to be critical. ${ }^{4,6}$ Several authors have suggested that interventions are likely to have greater effect when targeting people at risk. ${ }^{7,8}$ People who attend an A\&E department with an injurious fall form a high-risk group, and are expected to be more receptive to an intervention programme aimed at reducing falls than the general population of community-dwelling elderly people. In a study by Close et al., about half of the patients who attended an A\&E department with a fall had experienced an earlier fall in the previous year, compared to about one third of the elderly people in the general population. ${ }^{5}$ Like Close et al., ${ }^{5}$ we chose community-dwelling elderly people aged 65 years or over who had sustained an injurious fall as the target population of our intervention programme. The following definition of a fall was used: 'A fall is an event which results in a person coming to rest inadvertently on the ground or other lower level'. This definition is derived from that used by the Kellogg International Work Group. ${ }^{9}$

\section{Recruitment of the study population}

Recruitment of subjects took place at the local GP cooperative and the A\&E department of the University Hospital in Maastricht. The Maastricht GP cooperative is a group of GPs from practices in the town of Maastricht and the surrounding area who have founded a non-profit organisation to provide care for their own patients after hours. ${ }^{10}$ The Maastricht GP cooperative has been set up at the hospital's A\&E department and covers the out-of-hours service for all local GPs. ${ }^{11}$

The following inclusion criteria were used: age 65 years or older, communitydwelling, having visited the A\&E department or GP cooperative at the University Hospital Maastricht for the consequences of a fall, and living in Maastricht or its surrounding area. People were only allowed to enter the programme after completing and returning an informed consent form. Exclusion criteria were: not able to speak or understand Dutch, not able to complete questionnaires or interviews by telephone, cognitive impairment (a score of less than 4 on the Abbreviated Mental Test 4 (AMT 4), ${ }^{12,13}$ long-term admission to a hospital or other institution (more than four weeks from the date of inclusion), permanently bedridden, or fully dependent on a wheelchair. 


\section{Sample size calculation}

Sample size calculations were based on the expected effects of the intervention on the main outcome measure, the percentage of people sustaining a fall during one year of follow-up. The study by Close et al. ${ }^{5}$ found that the percentage of persons who sustained a recurrent fall was $52 \%$ in the usual care group and $32 \%$ in the intervention group. If we want to detect the same reduction in the percentage of persons sustaining a recurrent fall in our study, with a power (1-beta) of $90 \%$ and alpha of 0.05 , we need 123 patients in each group (a total of 246). Based on the experiences of Close et al. and our own experiences in trials among elderly people in the Netherlands, ${ }^{6}$ we expect a dropout rate of about $25 \%$ during the one-year follow-up period. This means that about 164 persons per group (a total of 328) have to be included in the study. The inclusion period was 14 months.

\section{Intervention programme}

To adapt the programme developed by Close et al. ${ }^{5}$ to the Dutch situation, and to make improvements based on recent insights, we performed a review of the literature, convened a consensus meeting and tested the adapted version in a pilot study $(n=36)$. Based on this process, we made some improvements to Close et al.'s programme.

The final programme includes a medical and occupational-therapy assessment resulting in recommendations. The medical assessment consists of an examination performed by a geriatrician, a geriatric nurse and a rehabilitation physician to identify and address risk factors for falling. The examination includes a comprehensive general examination, but in addition focussed on a more detailed assessment of visual acuity, stereoscopic vision, mobility, balance, cognition, affect, use of medication and examination of feet and footwear. Recommendations or indications for referral resulting from this examination are sent to the patient's GP. After the medical assessments, an occupational therapist visits the patients to identify possible risk factors for falling in the home environment. The therapist makes recommendations regarding adaptations to the home environment, assistive devices, home care and behavioural change. Recommendations by the occupational therapist are sent directly to the subjects themselves and to their GPs. As stated before, the intervention period is scheduled to last for a maximum of 3.5 months after the baseline measurement. 
An important addition to Close et al.'s protocol ${ }^{5}$ is the collaboration with a rehabilitation physician (physiatrist) in the medical part of the intervention. In addition to the screening by a geriatrician, our programme also involves screening by a rehabilitation physician who examines the subjects' feet and the shoes which the subjects wore at the time of the fall. Details of the process of adaptation and the contents of the intervention programme will be published elsewhere.

\section{Usual care in the Netherlands}

People in the control group receive usual care. At present, no guidelines exist for the systematic assessment of the underlying causes of an injurious fall presented at an A\&E department or GP cooperative in the Netherlands. In usual care, medical risks and other risk factors such as environmental hazards in the home and patients' risk behaviour are not systematically registered and addressed by hospital physicians, specialists or general practitioners. Moreover, no systematic attention is currently being paid to the specific consequences of an injurious fall for the daily functioning of individual patients in their unique situation. We placed no restrictions on co-interventions.

\section{Effect evaluation}

The primary outcome measures of the effect evaluation are number of falls and daily functioning. Number of falls is subdivided into three separate measures: the percentage of elderly people sustaining a fall during the oneyear follow-up period, recurrent falls during follow-up (i.e., the percentage of elderly people sustaining two or more falls), and injurious falls during followup (the percentage of elderly people receiving medical care after a fall). Falls are recorded continuously by means of a fall calendar during the twelvemonth follow-up period. Subjects are called monthly to report their falls as recorded on the fall calendar relating to the previous month.

We decided to measure daily functioning by means of the Frenchay Activity Index $(\mathrm{FAI}){ }^{14}$ in contrast to Close et al., ${ }^{5}$ who used the Barthel Index. Our reason for choosing this instrument was that the FAI has proved to be suitable for the general population of elderly people ${ }^{6}$ and has at least two advantages over the Barthel Index. One is that the Barthel Index shows a ceiling effect when applied to elderly people who have sustained a fall. ${ }^{5}$ The other is that most activities of daily living (ADL) scales, like the Barthel Index, do not refer to complex activities like housekeeping, recreation, hobbies and social 
interaction. These so-called instrumental abilities (IADL) may affect the quality of life considerably, and the FAI focuses primarily on these IADL abilities. ${ }^{14}$ The FAI is measured by means of self-administered questionnaires at baseline and after four and twelve months.

Our secondary outcome measures are: recuperation from the fall, health complaints, perceived health measured by means of the first two items of the RAND-36, ${ }^{15}$ ADL and IADL disability measured by means of the GARS (Groningen Activity Restriction Scale), ${ }^{16}$ mental health measured by means of the HADS (Hospital Anxiety and Depression Scale) ${ }^{17,18}$ and quality of life measured by means of the European Quality of Life instrument (EuroQol) ${ }^{19}$. The secondary outcome measures are assessed by means of self-administered questionnaires at four and twelve months.

Besides the primary and secondary outcome measures, we assess some background variables which are considered to be predictors, confounders or effect modifiers. The following personal characteristics are assessed: age, sex, marital status, living alone and socio-economic status. In addition, we assess the circumstances and causes of the falls reported at the GP cooperative and/or A\&E department, the consequences of the falls (using the Falls Handicap Inventory), ${ }^{20}$ the type of injury, falls in the previous year (retrospective), the patient's height, weight, use of medication and social contacts (using an adjusted version of items 4 and 5 of the Rand Social Health Battery), ${ }^{21,22}$ and the occurrence of life events. All background variables are measured at baseline.

\section{Economic evaluation}

The economic evaluation in our study is being performed from a societal perspective, which implies that all costs and outcomes are taken into account if possible. The economic evaluation will be a combination of a costeffectiveness and a cost-utility analysis. The primary outcome measure for the cost-effectiveness analysis will be the percentage of people sustaining a fall during one year of follow-up. As mentioned above, falls are recorded by means of a calendar. Within the cost-utility analysis, the effects are measured in terms of generic health-related quality of life descriptions, measured according to the standard Dutch version of the EuroQol (EQ 5-D) ${ }^{19}$ in selfadministered questionnaires at baseline and after four and twelve months. A direct value for every state of health is generated using the social tariff, ${ }^{23}$ 
which involves an algorithm for interpolating EuroQol results to population utilities.

We will assess programme costs, healthcare costs and patient and family costs. All costs are measured by means of a cost diary, ${ }^{24}$ in which patients continuously record volumes of healthcare utilization during the twelvemonth follow-up period. Subjects are asked to report their cost diary relating to the previous month during the same monthly telephone interview in which they report falls from the calendar. The volume of each category we measure will be multiplied by the cost price of each category. Cost prices are presented in Euros. Healthcare costs are estimated according to the Dutch guideline for cost analysis in healthcare research. ${ }^{25}$ Where such guidelines are not available for a specific category, real costs or tariffs are used to estimate costs.

\section{Process evaluation}

The process evaluation involves assessing the extent to which the intervention programme is performed according to protocol, the nature of the recommendations made to the participants, participants' compliance with these recommendations and the opinions of participants, physicians and therapists about the intervention programme and recommendations. Data on these topics are collected using the following methods: structured registration forms for the medical and occupational parts of the intervention programme; self-administered evaluation forms filled in by the participants after the medical intervention; interviews by telephone with the participants six weeks or longer after the recommendations are sent and interviews with all participating physicians and therapists at the end of the intervention period.

\section{Analysis}

Data will be primarily analysed according to the intention-to-treat principle, i.e., including all participants with valid data, regardless of whether they received or did not receive the intervention. Subsequently, the results of the intention-to-treat analysis will be compared with the results of an ontreatment analysis, to assess whether protocol deviations have caused bias. Participants with documented deviations from the study protocol (i.e., participants in the intervention group who did not receive the entire intervention or participants in either the intervention or the control group with incomplete follow-up data) will be excluded from this on-treatment analysis. 
Comparability between the intervention and control groups will be assessed at baseline to check for differences between the two groups. Outcome at four and twelve months will be compared between the intervention and control groups by both univariate and multivariate techniques. We will use multivariate analysis to adjust for possible differences in baseline scores and background variables between the intervention and control groups. Dropouts and losses-to-follow up will be described.

The economic evaluation will involve calculating cost-effectiveness and costutility ratios. The additional costs and additional benefits of the intervention programme compared with usual care will be examined by calculating incremental cost-effectiveness and cost-utility ratios. These incremental ratios represent the difference in mean costs between the intervention and usual care groups in the numerator and the difference in mean effects in the denominator. ${ }^{26}$

Since the recruitment period is only 14 months, and the follow-up period is also relatively short (12 months), it is unlikely that there will be substantial differences between costs made by and for patients who started in the first part of the recruitment period and those who started in the last part. Therefore, discounting of costs is not required. Finally, a sensitivity analysis will be done to assess the generalisability of the assumptions made in the costing process. This sensitivity analysis, which involves calculating the upper and lower limits of the confidence interval of cost and effect variables, will allow us to explore and quantify the uncertainty not related to sampling variations.

The process evaluation will mainly be analysed by means of descriptive techniques.

\section{Progress of the study}

Recruitment of eligible subjects commenced in December 2002 and ended in February 2004, resulting in a total of 333 eligible subjects being included in the trial. Randomization resulted in the allocation of 166 participants to the intervention group and 167 to the control group. Of the 333 persons recruited, $105(32 \%)$ are male and $228(68 \%)$ are female.

The follow-up period is to end in May 2005. Results on the effects of the programme will be available in 2006 and will be published in the relevant journals. 


\section{DISCUSSION}

Although the intervention has been subject of earlier research, this study will provide new information about the effectiveness in the Dutch situation. Furthermore, the results of the economic evaluation can provide information about the cost-effectiveness of the intervention and the effects on quality of life. In case of shown effectiveness and cost-effectiveness, we aim to implement this intervention into usual healthcare.

\section{ACKNOWLEDGEMENTS}

This study was funded by the Netherlands Organisation for Health Research and Development (ZonMw), grant number 945-02-053.

\section{REFERENCES}

1. Godlee F. Publishing study protocols: making them visible will improve registration, reporting and recruitment. BMC News and Views 2001;2:4.

2. Thornton A, Lee P. Publication bias in meta-analysis: its causes and consequences. J Clin Epidemiol 2000;53(2):207-16.

3. Steenstra IA, Anema JR, Bongers PM, de Vet HC, van Mechelen W. Cost effectiveness of a multi-stage return to work program for workers on sick leave due to low back pain, design of a population based controlled trial [ISRCTN60233560]. BMC Musculoskelet Disord 2003;4(1):26.

4. Gillespie LD, Gillespie WJ, Robertson MC, Lamb SE, Cumming RG, Rowe BH. Interventions for preventing falls in elderly people. Cochrane Database Syst Rev 2003(4):CD000340.

5. Close J, Ellis M, Hooper R, Glucksman E, Jackson S, Swift C. Prevention of falls in the elderly trial (PROFET): a randomised controlled trial. Lancet 1999;353(9147):93-97.

6. Dijcks BP, Neyens JC, Schols JM, van Haastregt JC, de Witte LP. [Falls in nursing homes: on average almost two per bed per year, resulting in a fracture in 1.3\%]. Ned Tijdschr Geneeskd 2005;149(19):1043-7.

7. Koch M, Gottschalk M, Baker DI, Palumbo S, Tinetti ME. An impairment and disability assessment and treatment protocol for community-living elderly persons. Phys Ther 1994; 74(4):286-94.

8. Cumming RG, Thomas M, Szonyi $G$, et al. Home visits by an occupational therapist for assessment and modification of environmental hazards: a randomized trial of falls prevention. J Am Geriatr Soc 1999;47(12):1397-402.

9. The prevention of falls in later life. Dan Med Bull 1987;34 Suppl 4:1-24.

10. Leibowitz R, Day S, Dunt D. A systematic review of the effect of different models of after-hours primary medical care services on clinical outcome, medical workload, and patient and GP satisfaction. Fam Pract 2003;20(3):311-7.

11. Van Uden CJ, Winkens RA, Wesseling GJ, Crebolder HF, van Schayck CP. Use of out of hours services: a comparison between two organisations. Emerg Med J 2003;20(2):184-7.

12. Swain DG, Nightingale PG. Evaluation of a shortened version of the Abbreviated Mental Test in a series of elderly patients. Clin Rehabil 1997;11(3):243-8. 
13. Swain DG, O'Brien AG, Nightingale PG. Cognitive assessment in elderly patients admitted to hospital: the relationship between the shortened version of the Abbreviated Mental Test and the Abbreviated Mental Test and Mini-Mental State Examination. Clin Rehabil 2000;14(6):608-10.

14. Schuling J, de Haan R, Limburg M, Groenier KH. The Frenchay Activities Index. Assessment of functional status in stroke patients. Stroke 1993;24(8):1173-7.

15. Van der Zee KI, Sanderman R. Het meten van de algemene gezondheidstoestand met de RAND-36: een handleiding [Measuring general health with the RAND-36:manual]. Groningen: Noordelijk Centrum voor Gezondheidsvraagstukken, Rijksuniversiteit Groningen, 1993.

16. Kempen GI, Miedema I, Ormel J, Molenaar W. The assessment of disability with the Groningen Activity Restriction Scale. Conceptual framework and psychometric properties. Soc Sci Med 1996;43(11):1601-10.

17. Bjelland I, Dahl AA, Haug TT, Neckelmann D. The validity of the Hospital Anxiety and Depression Scale. An updated literature review. J Psychosom Res 2002;52(2):69-77.

18. Flint AJ, Rifat SL. Factor structure of the hospital anxiety and depression scale in older patients with major depression. Int J Geriatr Psychiatry 2002;17(2):117-23.

19. Brooks R. Euroqol:the current state of play. Health policy 1996;37(1):53-72.

20. Rai GS, Kiniorns M, Wientjes H. Falls Handicap Inventory (FHI)--an instrument to measure handicaps associated with repeated falls. J Am Geriatr Soc 1995;43(6):723-4.

21. Donald CA, Ware JE, Brook RH, Davies-Avery A. Conceptualisation and measurement of health for adults in the Health Insurance Study. Santa Monica, CA: The rand Corporation, 1978.

22. McDowell I, Newell C. Measuring Health: a guide to rating scales and questionnaires. New York: Oxford University Press, 1996.

23. Dolan P. Modeling valuations for EuroQol health states. Med Care 1997;35(11):1095-108.

24. Goossens ME, Rutten van Molken MP, Vlaeyen JW, van der Linden SM. The cost diary: a method to measure direct and indirect costs in cost-effectiveness research. J Clin Epidemiol 2000;53(7):688-95.

25. Oostenbrink JB, Koopmanschap MA, Rutten FFH. Handleiding voor kosten onderzoek; methoden en richtlijnprijzen voor economische evaluaties in de gezondheidszorg. Amstelveen: College voor zorgverzekeringen, 2000.

26. Drummond MF, O'Brien BJ, Stoddart GL, Torrance GW. Methods for the Economic Evaluation of Health Care Programmes. New York: Oxford University Press, 2003. 



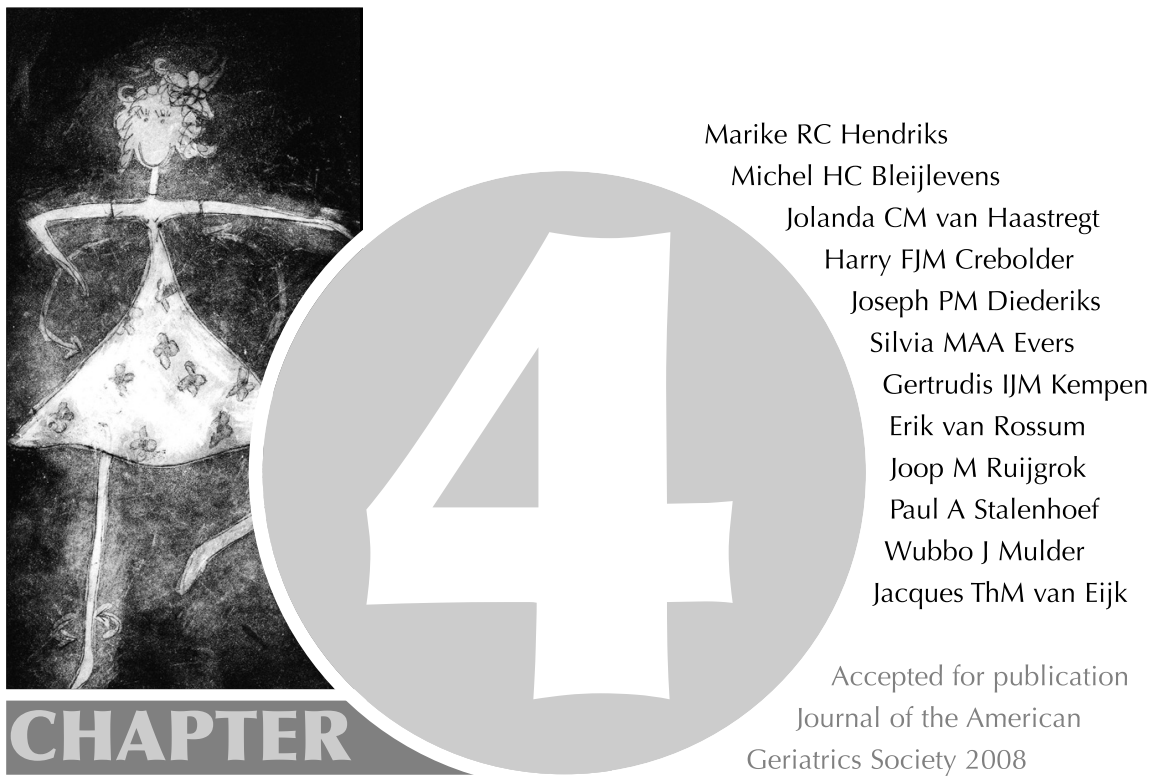




\section{ABSTRACT}

\section{Objective}

We adapted a successful British fall prevention programme to Dutch healthcare and assessed whether this pragmatic multidisciplinary programme was more effective than usual care in preventing new falls and functional decline among elderly people.

Design

A two-group randomized controlled trial with 12 months of follow-up.

Setting

University hospital and home-based intervention, the Netherlands.

Participants

333 community-dwelling Dutch elderly people aged 65 years or over who were seen at an accident and emergency department after a fall.

Intervention

Participants in the intervention group underwent a detailed medical and occupationaltherapy assessment to evaluate and address risk factors for recurrent falls, followed by recommendations and referral if indicated. People in the control group received usual care.

Measurements

Number of people sustaining a fall (fall calendar) and daily functioning (Frenchay Activity Index).

Results

Results showed no statistically significant favorable effects on falls (Odds Ratio $=0.86,95 \%$ Confidence Interval $(\mathrm{Cl})=0.50$ to 1.49 ) or daily functioning (Regression Coefficient $=0.37, \mathrm{CI}$ $=-0.90$ to 1.63) after twelve months of follow-up.

\section{Conclusion}

The multidisciplinary fall prevention programme is not effective in preventing falls and functional decline in this Dutch healthcare setting. We do not recommend implementing the programme in its present form in the Netherlands. Our trial shows that there can be considerable discrepancy between the "ideal" (experimental) version of a programme and the implemented version of the same programme. We therefore stress the importance of implementation research assessing feasibility and effectiveness of such a programme in a specific healthcare setting. 


\section{INTRODUCTION}

Falls are common occurrences among elderly people worldwide and may have several adverse consequences, such as physical injuries and psychological distress, leading to decreased functioning and quality of life. ${ }^{1,2}$ Injurious falls in particular are associated with an increase in healthcare utilization. ${ }^{3}$ The need for effective fall prevention strategies is thus evident. There is now considerable evidence for the effectiveness of multidisciplinary and multifactorial interventions that screen for health and environmental risk factors and address these factors. ${ }^{1,4,5}$ Close and colleagues developed a medical and occupational-therapy assessment for elderly persons attending an accident and emergency department in the inner city of London (United Kingdom) after an injurious fall. This programme showed convincing favorable effects on falls and daily functioning. ${ }^{2}$ When a programme has been shown to be effective in an experimental setting, however, it is important to assess whether it is also effective when implemented as a part of routine healthcare. We therefore evaluated a version of this programme, adapted to the Dutch heath system, to assess its robustness and to ascertain whether it could be recommended for implementation in the Netherlands.

The objective of the current study was to assess whether a multidisciplinary fall prevention programme was more effective than usual care in preventing new falls and functional decline among community-dwelling Dutch elderly people who attended an accident and emergency department (A\&E department) after a fall. In this study a fall is defined as an event that results in a person coming to rest inadvertently on the ground or other lower level, ${ }^{6}$ and an injurious fall as a fall for which medical care was sought.

\section{METHODS}

\section{Study design and participants}

The study design was a two-group randomized controlled trial with 12 months of follow-up. Outcomes were measured at four and twelve months after baseline. Sample size calculations indicated that 164 persons per group had to be included to detect the same reduction in falls as was found by Close and colleagues (52\% fallers in the usual care group and $32 \%$ fallers in the interven- 
tion group), with a power of $90 \%$, an alpha of 0.05 , and an expected dropout rate of $25 \%$ during 12 months of follow-up. ${ }^{2,7}$ Our study included communitydwelling elderly people aged 65 years and over who attended the A\&E department of the University Hospital Maastricht for the consequences of a fall. Since the out-of-hours accident and emergency care in Maastricht is carried out by the General Practitioner's Cooperative (GP Cooperative) ${ }^{8}$ participants were also recruited at the GP Cooperative. People were excluded if they were unable to speak Dutch, were cognitively impaired (a score of less than 4 on the Abbreviated Mental Test 4), ${ }^{9}$ had been admitted for more than 4 weeks to a hospital or another institution, and/or were permanently wheelchairdependent or bedridden. Eligible participants who signed the informed consent form and returned a completed baseline questionnaire were allocated to the intervention or control group by computerized alternate random allocation by an external agency. Participants allocated to the intervention group underwent medical and occupational-therapy assessments, followed by recommendations or further referral if indicated. The control group received usual care only. No restrictions were placed on co-interventions. To ensure blinding during data collection, measurements by phone were contracted out to an independent call centre (Centre for Data and Information Management, MEMIC), whose operators were unaware of group allocation. The design was approved by the Medical Ethics Committee of the Maastricht University and University Hospital, and is described in detail elsewhere. ${ }^{7}$

\section{Intervention}

\section{Fall prevention programme}

We adapted the programme developed by Close and colleagues ${ }^{2}$ to make it implementable in the Dutch setting, and subsequently assessed its feasibility in a pilot study. The feasibility study revealed that some adaptations programme adaptations were necessary to increase the chance of successful implementation in the Netherlands. The adaptation process and feasibility study are described elsewhere. ${ }^{10}$ Briefly, the adapted programme consisted of structured medical and occupational-therapy assessments to assess and address potential risk factors for new falls. The medical and occupational assessments were described in a protocol. The medical assessments were performed at the Maastricht University Hospital and comprised a comprehensive general examination, and a more detailed assessment of vision, ${ }^{11,12}$ sense of hearing, locomotor apparatus, ${ }^{13}$ feet and footwear, ${ }^{14}$ peripheral nervous system, balance and mobility (Romberg and Get Up and Go Test), ${ }^{15,16}$ anthropometry, cognition (Mini Mental State Examination), ${ }^{17}$ affect (Geriatric De- 
pression Scale), ${ }^{18}$ blood tests if indicated and medication use. Table 1 presents details about the assessments.

Table 1 Medical and occupational-therapy assessments

\begin{tabular}{|c|c|}
\hline \multicolumn{2}{|l|}{ Medical Assessment } \\
\hline Assessments & Details/Tools/ Test Batteries \\
\hline Standard examination & $\begin{array}{l}\text { Anamnesis and fall history } \\
\text { Cardiovascular, respiratory, abdominal system } \\
\text { and neurological system }\end{array}$ \\
\hline Blood pressure & Supine and erect \\
\hline Vision & $\begin{array}{l}\text { Visual acuity (Snellen) }{ }^{11} \\
\text { Visual fields (confrontation) } \\
\text { Stereoscopic vision }^{12}\end{array}$ \\
\hline Sense of hearing & Whispered voice test \\
\hline $\begin{array}{l}\text { Locomotor apparatus: lumbar spine, upper } \\
\text { and lower extremities }\end{array}$ & $\begin{array}{l}\text { Tone \& Power (MRC Scale) }{ }^{13} \\
\text { Joint deformity } \\
\text { Range of movement } \\
\text { Handgrip dynamometry }\end{array}$ \\
\hline Feet and footwear & $\begin{array}{l}\text { Callus, skin ulcers, oedema and arterial } \\
\text { pulsations } \\
\text { Footwear assessment form }{ }^{14}\end{array}$ \\
\hline Peripheral nervous system & $\begin{array}{l}\text { Sensation (monofilaments) } \\
\text { Vibration (tuning fork) } \\
\text { Proprioception (great toe) }\end{array}$ \\
\hline Balance and mobility & $\begin{array}{l}\text { Romberg } \\
\text { Get Up and Go Test } \\
{ }^{16}\end{array}$ \\
\hline Anthropometric indices & $\begin{array}{l}\text { Body weight }(\mathrm{kg}) \\
\text { Height }(\mathrm{m})\end{array}$ \\
\hline Cognition & Mini Mental State Examination ${ }^{17}$ \\
\hline Affect & Geriatric Depression Scale ${ }^{18}$ \\
\hline Heart & Electrocardiogram \\
\hline Blood tests & If indicated \\
\hline Medication & Prescribed medication list \\
\hline Occupational-therapy assessment & \\
\hline Assessments & Test Battery \\
\hline Function assessment & $\begin{array}{l}\text { Frenchay Activity Index }{ }^{19} \\
\text { Checklist of the local agency deciding on } \\
\text { eligibility for care, aids and devices }{ }^{20,21}\end{array}$ \\
\hline $\begin{array}{l}\text { Environmental hazards } \\
\text { Psychological consequences of the fall }\end{array}$ & $\begin{array}{l}\text { Dutch version of the Home Checklist }{ }^{22} \\
\text { Falls Handicap Inventory }\end{array}$ \\
\hline
\end{tabular}

The medical assessment in the British programme was performed by a geriatrician working in a day hospital who was at the same time the main researcher of the trial evaluating the programme. In the British setting, the geriatrician could directly refer patients to other relevant disciplines. In the Netherlands, this approach would not fit in with regular care, because the geriatrician is not a member of the A\&E department staff and non-acute 
referrals to medical specialists have to be made via the participants' GP (otherwise health insurance companies will not cover the costs of these referrals). ${ }^{19}$ In addition, GPs are familiar with the health status of their patients and can act as supervisors to provide the best possible care. We therefore decided to incorporate the GPs in the programme by sending them a summary of the results of the medical assessments, written by the geriatrician. If necessary, this summary also included recommendations and/or referrals to relevant services. Participants were recommended to contact their GP about these results, recommendations, and/or referrals following the medical assessment. The GPs could then take action if they agreed with the recommendations and/or referrals. As the geriatrician is usually assisted by a geriatric nurse in the Netherlands, we also incorporated a geriatric nurse to do the less complex assessments. In addition, a rehabilitation physician was included, because rehabilitation physicians are more specialized in examining feet and footwear ${ }^{14}$ than geriatricians.

After the medical assessment, an occupational therapist visited the participants at home for a structured functional and environmental assessment. Daily functioning was assessed by the 15-item Frenchay Activity Index (FAI), ${ }^{20}$ and an occupational-therapy checklist. ${ }^{21,22}$ Environmental hazards were identified and registered by means of a home-safety checklist. ${ }^{23}$ In addition, the Falls Handicap Inventory $(\mathrm{FHI})^{24}$ was used to assess handicaps associated with repeated falls. The participants received recommendations with regard to behavioural change, functional needs and safety within the home environment. Technical aids and adaptations or additional support were directly referred to and delivered by social and community services. To increase compliance, participants were sent a letter with the recommendations and/or referrals from the occupational therapist, by way of reminder. A copy of this letter was sent to the participants' GP.

The medical assessment was scheduled to take place in the first month after baseline. Subsequently, the home assessment was scheduled within 1 month after the medical assessment. Afterwards, a summary of the results and recommendations for further referral were sent to the participants' GP. Therefore, it was expected to take at about 2.5 months (with a maximum 3.5 months) after baseline measurement for all recommendations to reach the participants and be implemented. 


\section{Usual care}

Currently, no standard approach to fall risk assessment is available for fallers presenting to the A\&E department and being discharged home. ${ }^{25}$ In usual care in the Netherlands, medical risks and other risk factors for falls, such as environmental hazards in the home and patients' risk behaviour, are not systematically recorded and addressed by hospital physicians, specialists or general practitioners. Moreover, when people present to the A\&E department or the GP cooperative, no systematic attention is usually given to the specific consequences of injurious falls for the daily functioning of individual patients in their unique situation.

\section{Measurements}

The primary outcome measures were falls (i.e. falls, recurrent falls, injurious falls and time to first fall) and daily functioning. Falls were measured as the percentage of participants sustaining at least one fall during the follow-up, recurrent falls as the percentage of participants sustaining two or more falls during follow-up and injurious falls as the percentage of participants who sought medical care after a fall. Time to the first fall was measured in weeks. Participants recorded their falls continuously on a fall calendar during twelve months after baseline. They were contacted monthly by telephone by an independent call centre (MEMIC) to report the falls noted on the calendar. Daily functioning was measured by means of the Frenchay Activity Index ${ }^{20}$ in a self-administered questionnaire at baseline and after four and twelve months.

Secondary outcome measures were: recuperation from the index fall ( 1 item), health complaints (19 items), perceived health (first item of the RAND-36), ${ }^{26}$ activities of daily living (ADL) and instrumental ADL disability (Groningen Activity Restriction Scale), ${ }^{27}$ mental health (Hospital Anxiety and Depression Scale), ${ }^{28}$ fear of falling ( 1 item), activity avoidance ( 1 item), social participation (2 items), and quality of life (EuroQol converted into utilities according to Dolans' tariffs). ${ }^{29,30}$ These outcomes were also measured by means of selfadministered questionnaires at baseline and after four and twelve months.

At baseline, we assessed the following background characteristics: age, gender, living situation (living alone versus not living alone), level of education (primary school or less versus more than primary school), self-reported weight, type of injury resulting from the index fall (fracture or joint dislocation, i.e. major injury, versus minor injury), handicaps associated with the index fall (Falls Handicap Inventory), ${ }^{24}$ and number of illnesses (20 items). 


\section{Analysis}

SPSS statistical software (version 13) was used for the analyses. Baseline characteristics of the intervention and control groups were analyzed by means of descriptive statistics. Short-term and long-term effects were analyzed according to the intention-to-treat principle, including all participants with valid data, regardless of whether they received the programme or not. Differences in outcomes between the groups were analyzed by means of multiple linear and logistic regressions and were adjusted for potential differences in covariates measured at baseline (i.e. the relevant outcome measure, age, gender, living situation, education, injury from the index fall, weight, illnesses, recurrent falls, and psychological consequences of the index fall). Survival analysis by means of Cox Proportional Hazards regression was used to study the time to first fall for each participant. In addition, per protocol analyses were performed on primary outcomes (at twelve months) for those participants in the intervention group who received the complete medical and occupational-therapy assessments. Subgroup analyses were performed with groups considered to be at increased fall risk, ${ }^{31,32}$ viz. people with a history of more than one fall in the previous year, people with mobility impairments (defined as reporting some problems with walking, or worse, on the mobility item of the EuroQol), ${ }^{29}$ poor ADL functioning (defined as a score below 30 on the FAI), ${ }^{20}$ and older age (80 years and older). Per-protocol and subgroup analyses were adjusted for the same covariates as the intention-totreat analysis.

Analyses of falls and recurrent falls included persons for whom at least 9 of the 12 months of the fall calendar data were available. Missing data were replaced by the individual mean of valid data. Injurious falls were analyzed by means of complete case analysis, because imputation of the data would lead to underreporting of injurious falls. In addition, we performed a sensitivity analysis to check whether analyses with those participants with complete data on all 12 fall calendar months (complete case analyses) would result in other conclusions about effectiveness. 


\section{RESULTS}

\section{Participants}

A detailed overview of the progress of participants during the trial is shown in figure 1. From January 2003 until March 2004, 2362 persons were recruited after they had attended the A\&E department and/or GP cooperative because of a fall. A majority of them did not meet the inclusion criteria $(n=774,38 \%)$ or refused to participate (e.g. not interested, too time consuming; $n=531,26 \%$ ). Finally, 333 participants gave informed consent and were randomly allocated to the intervention $(n=166)$ or control group $(n=167)$. After 12 months of follow-up, $25 \%(n=42)$ of the participants had dropped out of the intervention group, and $20 \%(n=33)$ out of the control group. The main reason for dropping out of the study was health problems. Reasons for dropout were similar in both groups.

Table 2 shows the baseline characteristics of the study population. Randomization achieved roughly equal balance between the groups.

The 75 participants who withdrew from the study during the twelve-month follow-up period were on average older (77.2 versus 74.2 years, $p=0.000$ ), had lower scores on the $\mathrm{FAI}$ at baseline (people who withdrew being more inactive, 20.8 versus 24.2, $\mathrm{p}=0.002$ ), and had higher scores on the $\mathrm{FHI}$ at baseline (participants who withdrew reporting more impairments associated with repeated falls, 30.7 versus $20.2, p=0.000)$. In addition, participants who withdrew reported more illnesses at baseline than those who completed the study (3.5 versus 2.9, $\mathrm{p}=0.033$; not tabulated). Education, income, and living situation of dropouts and completers were comparable. After the twelvemonth follow-up period, the intervention and control groups remained comparable in terms of background characteristics, except for level of education (data not tabulated). Drop-outs of the intervention group showed a higher percentage of low educated participants compared to completers $(38 \%$ versus $21 \%)$. After four and twelve months, data on $274(80 \%)$ and $258(77 \%)$ participants, respectively, were available for intention-to-treat analysis. 


\section{2}

CHAPTER 4

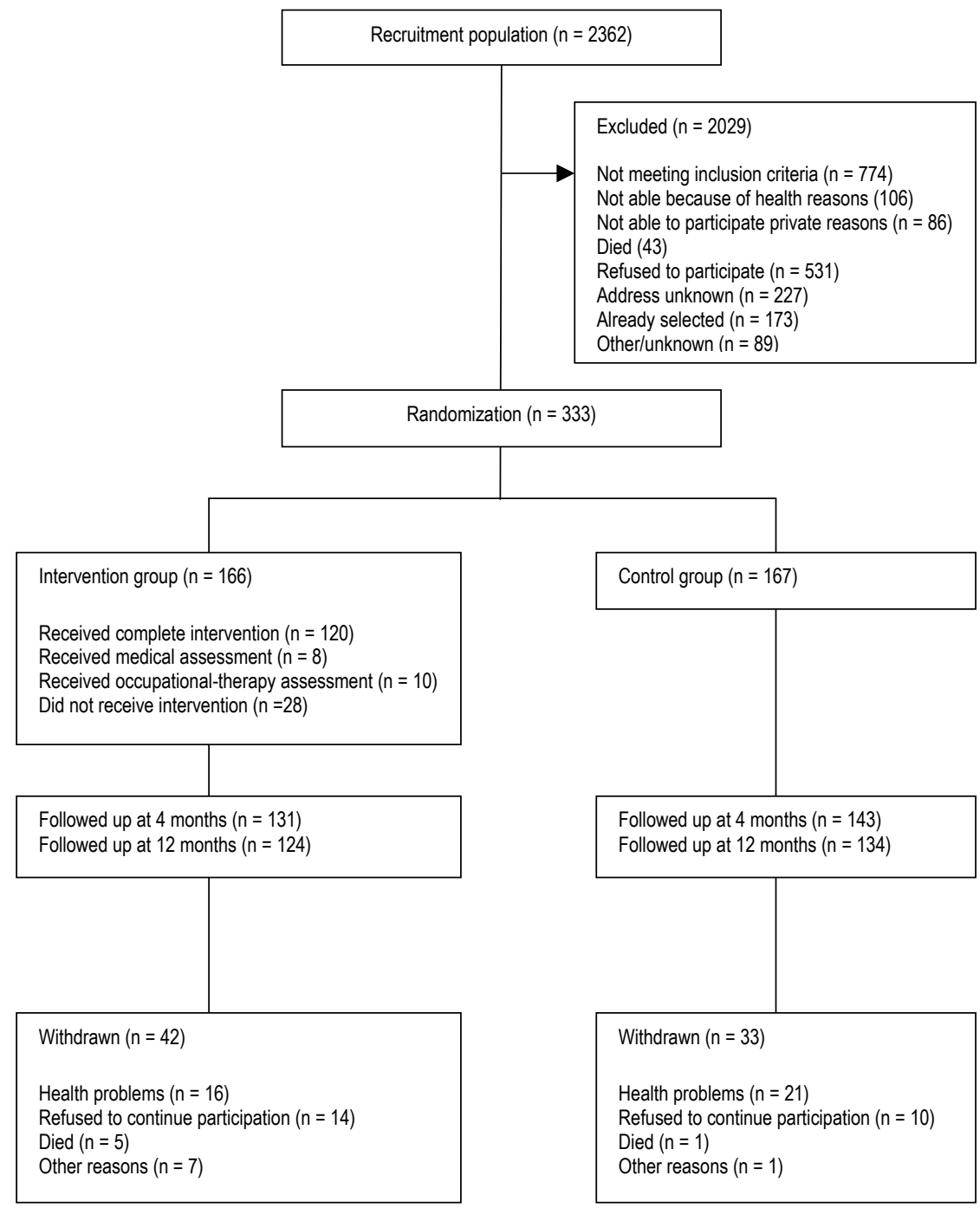

Figure 1 Flow of participants 
Table 2 Background characteristics and outcome measures at baseline for the intervention and control groups*

\begin{tabular}{|c|c|c|}
\hline \multirow{2}{*}{ Background characteristics } & $\begin{array}{l}\text { Intervention group } \\
\qquad(\mathrm{n}=166)\end{array}$ & $\begin{array}{l}\text { Usual care group } \\
\qquad(\mathrm{n}=167)\end{array}$ \\
\hline & & \\
\hline Mean (sd) age & $74.5(5.9)$ & $75.2(6.9)$ \\
\hline Female & 111 (66.9) & $117(70.1)$ \\
\hline Living alone & $73(44.2)$ & $71(42.5)$ \\
\hline$\leq$ primary school education & $42(25.3)$ & $52(31.1)$ \\
\hline $\begin{array}{l}\text { Major injury from index fall (fracture or joint } \\
\text { dislocation) }\end{array}$ & $66 \quad(39.8)$ & $60 \quad(35.9)$ \\
\hline Mean (sd) self-reported weight & $73.4(13.7)$ & $71.8(12.3)$ \\
\hline $\begin{array}{l}\text { Mean (sd) psychological consequences of the fall } \\
\text { (Falls Handicap Inventory) }\end{array}$ & $22.4(20.7)$ & $22.8(19.6)$ \\
\hline Mean (sd) number of illnesses & $2.8(2.0)$ & $3.2(2.5)$ \\
\hline \multicolumn{3}{|l|}{ Outcome measures } \\
\hline $\begin{array}{l}\text { Persons who had sustained at least one fall in the } \\
\text { previous year }\end{array}$ & $166(100)$ & $167(100)$ \\
\hline $\begin{array}{l}\text { Persons who had sustained more than one fall in } \\
\text { the previous year }\end{array}$ & $82(49.4)$ & $82(49.1)$ \\
\hline $\begin{array}{l}\text { Mean (sd) daily functioning (Frenchay Activity } \\
\text { Index) }\end{array}$ & $23.2(8.7)$ & $23.7(8.6)$ \\
\hline
\end{tabular}

*values are numbers (percentages) unless stated otherwise

\section{Outcomes}

A total of $138(83 \%)$ of the participants in the intervention group had received at least one of the assessments: $120(72 \%)$ of the participants had received both the medical and occupational-therapy assessments, ten persons only the medical assessment, eight persons only the occupational. Ninety-seven percent of the items of both assessments were carried out according to protocol. The medical assessment and the home visit took place within an average of 5 and 10 weeks after baseline, respectively. The results and recommendations were sent to the GPs an average of 3.5 months after baseline. A total of 50 (intended) referrals and 25 recommendations resulted from the medical assessment and were included in the geriatrician's letter to the GP. Of those receiving the medical assessments $(n=130), 56$ participants $(43 \%)$ received at least one recommendation or referral. Of those receiving the occupational-therapy assessments $(n=128), 117$ participants $(91 \%)$ received 456 recommendations. Overall the assessments resulted in at least one referral or recommendation for $89 \%(n=123)$ of the participants who received at least one of the assessments $(n=138)$. The process evaluation ${ }^{33}$ revealed that only half of the participants asked their GP about the referrals and recommendations, and 
a quarter of them did not receive the intended referrals and recommendations from their GP. When the referrals and recommendations actually reached the participants, self-reported compliance with referrals and recommendations from the medical assessments was $75 \%$. The self-reported compliance with the recommendations the participants reported to have received from the occupational-therapy assessment was also $75 \%$.

No significant differences between the two groups were observed in terms of falls or daily functioning (table 3) after four and twelve months. The analysis of falls and recurrent falls for the time after the programme was implemented (five to twelve months), and the complete case analysis of falls and recurrent falls showed no significant differences between the groups either (data not tabulated).

Survival analyses showed no differences in time to first fall between the groups, neither for the total follow-up period (one to twelve months, $\operatorname{Exp}(B)=$ 1.08; $p=0.66$ ), nor for the time after the programme was implemented (five to twelve months, $\operatorname{Exp}(B)=0.79 ; p=0.30$, not tabulated).

As regards the secondary outcomes, we found no differences between the groups, neither at four nor at twelve months (table 4). No adverse events or side effects were reported.

Subsequently, per protocol analyses were performed with all participants in the intervention group who had received both the medical and occupationaltherapy assessments $(n=120 ; 72 \%$ ) versus the complete control group. These analyses showed no significant differences on any outcome measure (data not shown). Furthermore, subgroup analyses did not show any differences in terms of falls between the subgroups in the intervention and control groups (data not shown). Only daily functioning (FAI) significantly improved in participants aged 80 years and over allocated to the intervention group (control $n=29$ versus intervention $n=22 ; B=4.134 ; p=0.019$; not tabulated). 


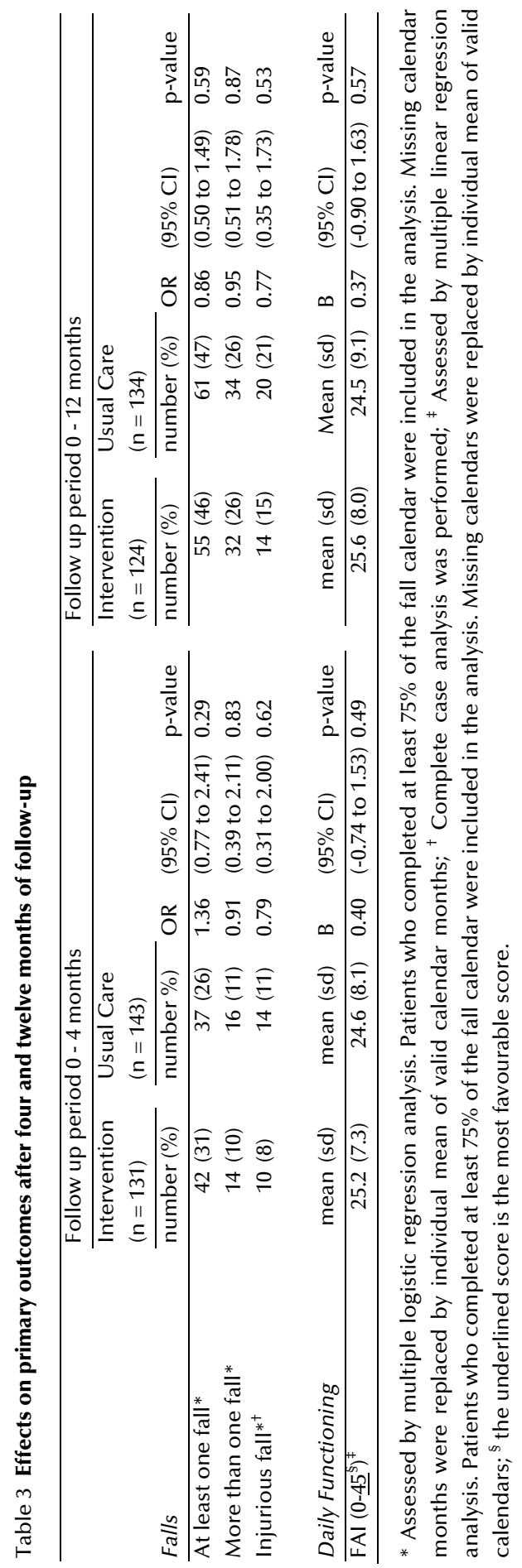




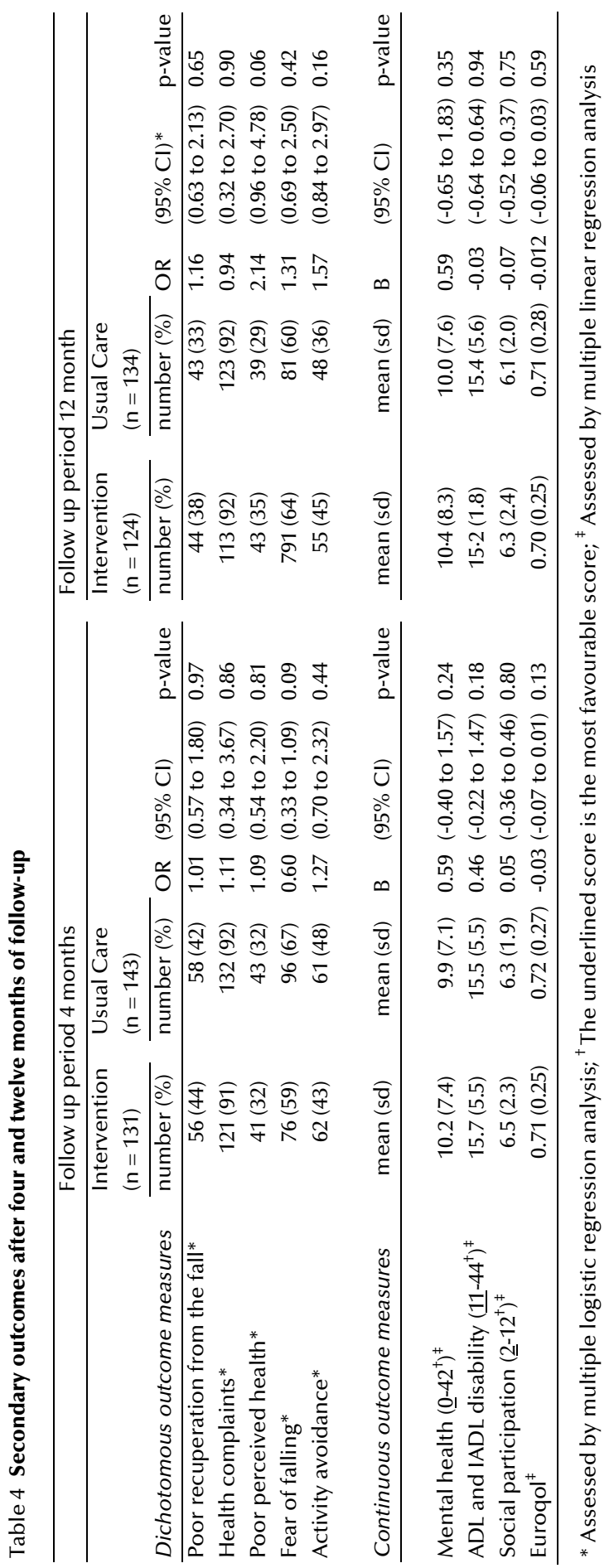




\section{CONCLUSION AND DISCUSSION}

We found no effect of our programme on falls, daily functioning or secondary outcome measures in our intention to treat analyses. Furthermore the analyses in which we compared those persons who received the complete intervention with the control group (per protocol analyses) did not show any effects in falls or daily functioning either. The same accounts for separate analyses of falls for the time after the programme was implemented (five to twelve months), and complete case analyses (including those participants with complete data). Our findings are in sharp contrast with those by Close and colleagues ${ }^{2}$ in the UK, who found that the multidisciplinary fall prevention programme on which we based our programme had favorable effects on the number of fallers and daily functioning. Furthermore, our results do not corroborate the conclusion by Gillespie and others that multifactorial risk assessment and management programmes generally appear to be effective in reducing falls. ${ }^{1,45}$ Gillespie and co-workers did note, however, that the effects are generally small, and they mention the problem of heterogeneity of results, indicating that this heterogeneity can probably be explained by differences in the status of the participants, the context, and details of the content and presentation of the intervention. ${ }^{1}$ Moreover, a very recent systematic review on multifactorial assessment and prevention programmes concluded that the evidence of benefit from these assessments and prevention programmes may be smaller than thought. ${ }^{34}$

There may be several explanations for the ineffectiveness of our programme and the marked difference between our results and those of the British trial. First, the results of our trial may have been influenced by the essential adaptations we made to the protocol, in order to integrate the programme as much as possible in routine Dutch healthcare. We added two disciplines (rehabilitation physician and geriatric nurse) to the medical assessment team and involved GPs in the programme. This resulted in an extended implementation period of about 3.5 months. This relatively long time between the fall and completion of the programme may have reduced the effectiveness of the programme. However, shortening this period would be a rather difficult without deviating from routine procedures in the Netherlands.

Second, some of the GPs involved in the trial had patients in both the intervention and the control groups in their practices. Therefore, it is possible that 
the programme influenced the way these GPs managed falls in their practices. However, because the referrals and the recommendations resulted from an individualized assessment we think this possible contamination effect was negligible. Moreover, the GPs were not aware of which patients participated in the control group.

Third, the differences between the outcomes of the two studies can be explained by differences in the trial populations. It is possible that patients enrolled in the British trial were more at risk of falls and/or more underserved and therefore more likely to benefit from the programme than the patients we assessed. Although we performed the same selection procedure, our study population was on average somewhat younger than the population selected for Close's study (74.9 versus 78.2 years). ${ }^{2}$ Moreover, more people died in the British study than in our study ( $n=46,12 \%$ versus $n=6,2 \%)^{2}$ indicating that the British sample consisted of more frail persons. However, the number of recurrent fallers in our control group was comparable to the findings of the control group of Close and other studies. ${ }^{2,35,36}$ Furthermore, subgroup analyses among participants who were older and more at risk did not reveal favorable effects of the programme on falls either. With regard to the available healthcare services, Thorbly described that London's health services do not sufficiently meet the needs of Londoners. ${ }^{37}$ This implies that the programme may have added more to routine care in London than to regular care in our Dutch setting. However, in our study in for $11 \%$ of the participants no further action was recommended, whereas in the study conducted by Close $16 \%$ of the participants required no further action. Close reported in her study that in only $24(16 \%)$ of the assessments no further action was required. ${ }^{2}$ Unfortunately, further comparison of the contents of the referral and recommendations resulting from the two trials is rather difficult, as Close does not report on the details of the referral or recommendations.

Fourth, the effectiveness of our programme may have been influenced by deviations from the protocol. A detailed process evaluation performed alongside the trial, however, revealed that our programme was largely performed according to protocol (with $97 \%$ of the protocol items performed as planned), and was considered to be feasible by those who administered the assessments. This makes it highly unlikely that deviations from protocol can explain the absence of favorable effects. 
Fifth, a lack of compliance with the programme may have influenced the outcomes. Only half of the participants who underwent the medical assessment actually called their GP, and a quarter of them did not receive the intended referrals and recommendations from their GP. Therefore many participants did not receive intended interventions. Recommending to participants that they contact their GP, and subsequently sending them a reminder was apparently not an effective way to make them call their GP. The fact that the GPs did not follow the geriatrician's suggestions in a quarter of the cases may have been caused by lack of time or differences of opinion, but also by overlap between the suggestions made by the geriatrician and the care and services already provided to these patients. ${ }^{38-41}$ The self-reported compliance with the referrals and recommendations that actually reached the participants was $75 \%$, which seems reasonably good. As Close and colleagues ${ }^{2}$ did not report on the compliance rate in their programme, it is not possible to compare the two studies in this respect.

Overall, we conclude that our multidisciplinary fall prevention programme in community-dwelling older people was not effective in the Dutch healthcare setting. Therefore, we do not recommend implementing this programme in its present form in usual care in the Netherlands. Involving the GP and extending the implementation period was in accordance with routine care in the Netherlands, but it may have reduced the effectiveness of the programme. There are some indications that higher intensity interventions that provide direct action (e.g. treatments) to address fall risk factors may be more effective than interventions that provide information and referral. ${ }^{34}$ Therefore, we recommend searching for possibilities to overcome the problem of non-acute referral, in order to increase the efficiency and effectiveness of the programme. Furthermore, mechanisms need to be found to enhance adherence of participants to the recommendations. Our study revealed that proven effectiveness of a prevention programme in one healthcare setting is no guarantee for its effectiveness in another setting. Moreover, there can be considerable discrepancy between an experimental version of a programme and the implemented version of the same programme. We therefore stress the importance of implementation research assessing feasibility and effectiveness of a programme in specific healthcare settings. To achieve effective fall prevention in practice, we need to study the barriers to implementation and try to overcome these problems. ${ }^{41}$ 


\section{ACKNOWLEDGEMENTS AND FUNDING}

We would like to thank the following persons for their contributions: Charlotte H. Mom-Schuurmann, CIZ Centre healthcare indications in the Netherlands; MEMIC, Centre for Data and Information Management; Maastricht University Hospital; Municipal authorities of Maastricht. This study was funded by the Netherlands Organization for Health Research and Development (ZonMw), grant number 945-02-053. P.O. Box 93 245, 2509 AE Den Haag, The Netherlands (Email info@zonmw.nl). The funding source had no involvement.

\section{REFERENCES}

1. Gillespie LD, Gillespie WJ, Robertson MC, Lamb SE, Cumming RG, Rowe BH. Interventions for preventing falls in elderly people. Cochrane Database Syst Rev 2003(4):CD000340.

2. Close J, Ellis M, Hooper R, Glucksman E, Jackson S, Swift C. Prevention of falls in the elderly trial (PROFET): a randomised controlled trial. Lancet 1999;353(9147):93-97.

3. Rizzo JA, Friedkin R, Williams CS, Nabors J, Acampora D, Tinetti ME. Health care utilization and costs in a Medicare population by fall status. Med Care 1998;36(8):1174-88.

4. Chang JT, Morton SC, Rubenstein LZ, et al. Interventions for the prevention of falls in older adults: systematic review and meta-analysis of randomised clinical trials. BMJ 2004; 328(7441):680.

5. Kannus $P$, Sievanen $H$, Palvanen $M$, Jarvinen $T$, Parkkari J. Prevention of falls and consequent injuries in elderly people. Lancet 2005;366(9500):1885-93.

6. The prevention of falls in later life. Dan Med Bull 1987;34 Suppl 4:1-24.

7. Hendriks MR, van Haastregt JC, Diederiks JP, Evers SM, Crebolder HF, van Eijk JT. Effectiveness and cost-effectiveness of a multidisciplinary intervention programme to prevent new falls and functional decline among elderly persons at risk: design of a replicated randomised controlled trial [ISRCTN64716113]. BMC Public Health 2005;5(1):6.

8. Van Uden CJ, Winkens RA, Wesseling GJ, Crebolder HF, van Schayck CP. Use of out of hours services: a comparison between two organisations. Emerg Med J 2003;20(2):184-7.

9. Swain DG, Nightingale PG. Evaluation of a shortened version of the Abbreviated Mental Test in a series of elderly patients. Clin Rehabil 1997;11(3):243-8.

10. Hendriks MR, Bleijlevens $M H$, van Haastregt JC, et al. A multidisciplinary fall prevention program for elderly persons: a feasibility study. Geriatric Nursing: accepted for publication; available upon request 2008:000-000.

11. Hetherington R. The Snellen chart as a test of visual acuity. Psychol Forsch 1954;24(4):349-57.

12. Walraven J. Amblyopia screening with random-dot stereograms. Am J Ophthalmol 1975;80(5): 893-900.

13. Van der Ploeg RJ, Oosterhuis HJ. Fysische diagnostiek--het meten van spierkracht. [Physical examination--measurement of muscle strength]. Ned Tijdschr Geneeskd 2001;145(1):19-23.

14. Menz HB, Sherrington C. The Footwear Assessment Form: a reliable clinical tool to assess footwear characteristics of relevance to postural stability in older adults. Clin Rehabil 2000; 14(6):657-64. 
15. Van Vaerenbergh J, Broos P. Positieve Romberg-test en het voorspellen van vallen bij bejaarden. [Positive Romberg test and the probability of falls in the aged]. Tijdschr Gerontol Geriatr 1990;21(2):71-4.

16. Mathias S, Nayak US, Isaacs B. Balance in elderly patients: the "get-up and go" test. Arch Phys Med Rehabil 1986;67(6):387-9.

17. Folstein MF, Folstein SE, McHugh PR. "Mini-mental state". A practical method for grading the cognitive state of patients for the clinician. J Psychiatr Res 1975;12(3):189-98.

18. Brink TL, Yesavage JA, Lum O, Heersema PH, Adey M, Rose TL. Screening tests for geriatric depression. Clin gerontologist 1982;1(1):37-43.

19. Kulu Glasgow I, Delnoij D, de Bakker D. Self-referral in a gatekeeping system: patients' reasons for skipping the general-practitioner. Health Policy 1998;45(3):221-38.

20. Schuling J, de Haan R, Limburg M, Groenier KH. The Frenchay Activities Index. Assessment of functional status in stroke patients. Stroke 1993;24(8):1173-7.

21. Rooken M, Van der Ven S. Standaard voor het afleggen van een huisbezoek door ergotherapeuten: Stroomschema en formulier. NVE-standaard. Utrecht: Nederlandse Vereniging voor Ergotherapie, 2001.

22. Ummels C, Lahaye V. Ergotherapeutische registratielijst voor een ADL-zelfverzorgingsobservatie: Handleiding en formulier. NVE-standaard. Utrecht: Nederlandse Vereniging voor Ergotherapie, 2001.

23. Stalenhoef P, Diederiks J, Knottnerus A, de Witte L, Crebolder H. How predictive is a homesafety checklist for indoor fall risk in the elderly living in the community? Eur J Gen Pract 1998; 4:114-120.

24. Rai GS, Kiniorns M, Wientjes H. Falls Handicap Inventory (FHI)--an instrument to measure handicaps associated with repeated falls. J Am Geriatr Soc 1995;43(6):723-4.

25. Russell MA, Hill KD, Blackberry I, Day LL, Dharmage SC. Falls risk and functional decline in older fallers discharged directly from emergency departments. J Gerontol A Biol Sci Med Sci 2006;61(10):1090-5.

26. Van der Zee KI, Sanderman R. Het meten van de algemene gezondheidstoestand met de RAND-36: een handleiding [Measuring general health with the RAND-36:manual]. Groningen: Noordelijk Centrum voor Gezondheidsvraagstukken, Rijksuniversiteit Groningen, 1993.

27. Kempen GI, Miedema I, Ormel J, Molenaar W. The assessment of disability with the Groningen Activity Restriction Scale. Conceptual framework and psychometric properties. Soc Sci Med 1996;43(11):1601-10.

28. Spinhoven P, Ormel J, Sloekers PP, Kempen GI, Speckens AE, Van Hemert AM. A validation study of the Hospital Anxiety and Depression Scale (HADS) in different groups of Dutch subjects. Psychol Med 1997;27(2):363-70.

29. Brooks R. Euroqol:the current state of play. Health policy 1996;37(1):53-72.

30. Dolan P. Modeling valuations for EuroQol health states. Med Care 1997;35(11):1095-108.

31. Tinetti ME, Speechley M, Ginter SF. Risk factors for falls among elderly persons living in the community. N Engl J Med 1988;319(26):1701-7.

32. Campbell AJ, Borrie MJ, Spears GF. Risk factors for falls in a community-based prospective study of people 70 years and older. J Gerontol 1989;44(4):M112-7.

33. Bleijlevens $M H$, Hendriks MR, van Haastregt JC, et al. Process factors explaining the ineffectiveness of a multidisciplinary fall prevention programme. Submitted available upon request 2008.

34. Gates S, Lamb SE, Fisher JD, Cooke MW, Carter YH. Multifactorial assessment and targeted intervention for preventing falls and injuries among older people in community and emergency care settings: systematic review and meta-analysis. BMJ 2008;336(7636):130-3.

35. Tinetti ME, Baker DI, McAvay G, et al. A multifactorial intervention to reduce the risk of falling among elderly people living in the community. N Engl J Med 1994;331(13):821-7. 
36. O'Loughlin J, Robitaille Y, Bolvin J-F, Suissa S. Incidence of and risk factors for falls and injurious falls among the community-dwelling elderly. Am J Epidemiol 1993;137:342-54.

37. Thorlby R, Dixon J, Dickson N. Health for London: showing England the way? BMJ 2007; 335(7611):108-9.

38. Yarnall KS, Pollak KI, Ostbye T, Krause KM, Michener JL. Primary care: is there enough time for prevention? Am J Public Health 2003;93(4):635-41.

39. Fortinsky RH, lannuzzi-Sucich M, Baker DI, et al. Fall-risk assessment and management in clinical practice: views from healthcare providers. J Am Geriatr Soc 2004;52(9):1522-6.

40. Chou WC, Tinetti ME, King MB, Irwin K, Fortinsky RH. Perceptions of physicians on the barriers and facilitators to integrating fall risk evaluation and management into practice. $J$ Gen Intern Med 2006;21(2):117-22.

41. Tinetti ME, Gordon C, Sogolow E, Lapin P, Bradley EH. Fall-risk evaluation and management: challenges in adopting geriatric care practices. Gerontologist 2006;46(6):717-25.

42. Blake AJ, Morgan K, Bendall MJ, et al. Falls by elderly people at home: prevalence and associated factors. Age Ageing 1988;17(6):365-72.

43. Rubenstein LZ. Falls in older people: epidemiology, risk factors and strategies for prevention. Age Ageing 2006;35 Suppl 2:ii37-ii41.

44. Todd C, Skelton D. What are the main risk factors for falls among older people and what are the most effective interventions to prevent falls? Copenhagen: WHO Regional Office for Europe (Health Evidence Network), 2004: 1-28. 
PROCESS FACTORS EXPLAINING THE INEFFECTIVENESS OF A MULTIDISCIPLINARY FALL PREVENTION PROGRAMME

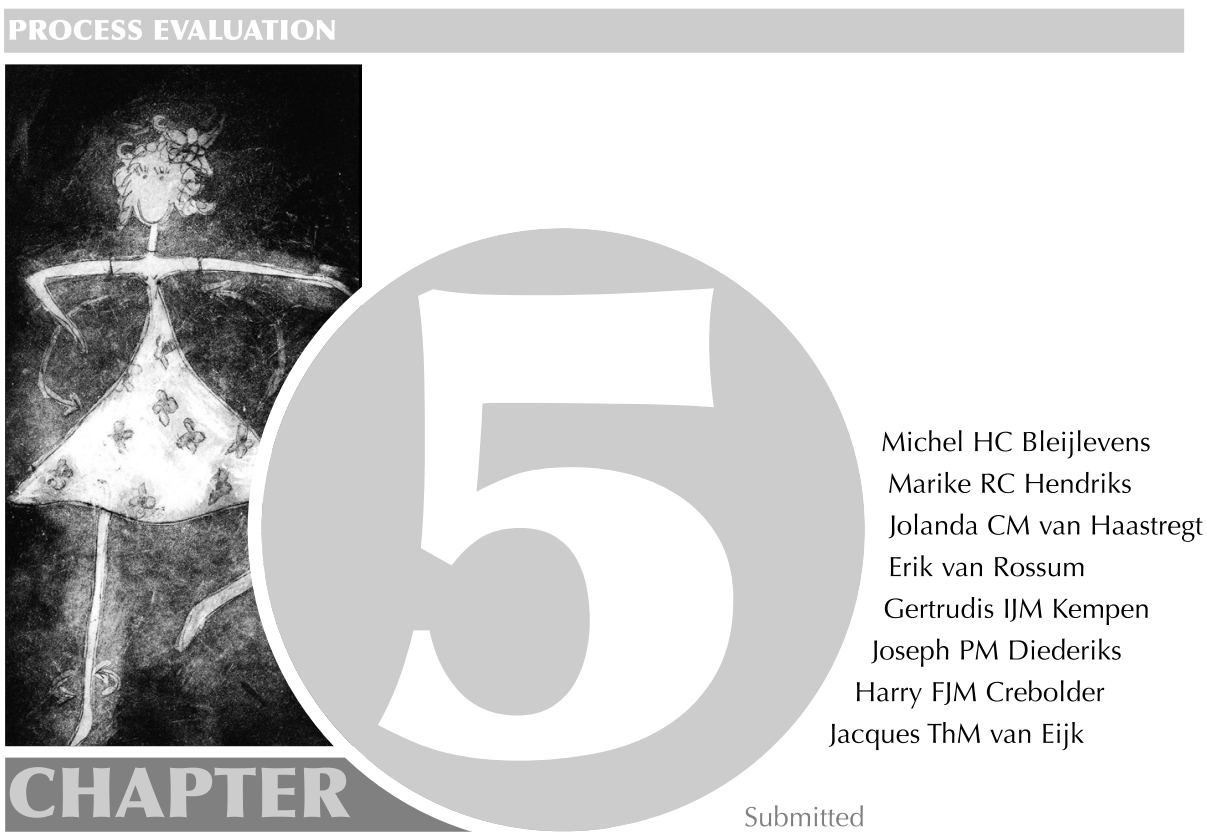




\section{ABSTRACT}

Background. Falls are a major health threat to older community-living people, and initiatives to prevent falls should be a public health priority. We evaluated a Dutch version of a successful British fall prevention programme. Results of this Dutch study showed no effects on falls or daily functioning. In parallel to the effect evaluation, we carried out a detailed process evaluation to assess the feasibility of our multidisciplinary assessment and referral programme. The present study reports on the results of this process evaluation.

Methods. The assessment and referral programme comprised a medical and occupational-therapy assessment, resulting in recommendations and/or referrals to other services if indicated. We used self-administered questionnaires, structured telephone interviews, structured recording forms, structured face-to-face interviews and a plenary group discussion to collect data from participants allocated to the intervention group $(n=166)$ and from all practitioners who performed the assessments $(n=8)$. The following outcomes were assessed: the extent to which the assessment and referral programme was performed according to protocol, the nature of the recommendations and referrals provided to the participants, participants' self-reported compliance and participants' and practitioners' opinions about the programme.

Results. Both participants and practitioners judged the programme to be feasible. The programme was largely performed according to protocol. The number of referrals and recommendations ensuing from the medical assessment was relatively small. Participants' self-reported compliance as regards contacting their GP to be informed of the recommendations and/or referrals ensuing from the medical assessment was low to moderate. However, self-reported compliance with such referrals and recommendations was reasonable to good. A large majority of participants reported they had benefited from the programme.

Conclusion. The results of the present study show that the assessment and referral programme was feasible for both practitioners and participants. The main factors that seem to be responsible for the lack of effectiveness are the relatively low number of referrals and recommendations ensuing from the medical assessments and participants' low compliance as regards contacting their GP about the results of the medical assessment. We do not recommend implementing the assessment and referral programme in its present form in regular care. 


\section{BACKGROUND}

Falls are a major health threat to older people living in the community, and initiatives to prevent these falls should be a public health priority. Approximately one third of community-dwelling people aged 65 and over fall at least once a year. ${ }^{1-6}$ About one fifth of all falls result in an injury that requires medical attention, and about one tenth lead to serious physical consequences, such as fractures, joint dislocations and lacerations. ${ }^{6-9}$ In addition, falls can have considerable psychosocial consequences, like fear of falling, depression and social isolation. ${ }^{10-12}$ Together, these physical and psychosocial consequences are responsible for reduced physical activity, ${ }^{11,13}$ early admission to hospital or nursing home, ${ }^{2,14}$ increased mortality and morbidity ${ }^{14,15}$ and loss of autonomy. ${ }^{2,10}$

Close and colleagues developed a multidisciplinary fall prevention programme aimed at community-dwelling people aged 65 years and over who had visited the accident and emergency (A\&E) department because of a fall. ${ }^{16,17}$ Although this programme showed promising effects in this British setting, this is no guarantee for its effectiveness in other healthcare settings. We therefore developed a Dutch version of this successful programme and tested its effect on falls and daily functioning by means of a randomized controlled trial. ${ }^{17}$ The results of this trial showed that the programme did not have any effect on falls or daily functioning. ${ }^{18}$ In parallel to this randomized controlled trial, we carried out a detailed process evaluation primarily aimed at assessing the feasibility of our multidisciplinary programme. The second aim of this process evaluation was to identify factors which might explain the lack of effectiveness of our programme. This paper presents the results of this process evaluation. We translated the two aims of our evaluation into the following four specific research questions:

1. To what extent was the fall prevention programme performed according to protocol?

2. What was the nature of the recommendations and referrals made to the participants?

3. What was the participants' self-reported compliance?

4. What are the participants' and practitioners' opinions about the programme? 


\section{METHODS}

\section{Fall prevention programme}

The fall prevention programme consisted of a medical and occupationaltherapy assessment, followed by recommendations or further referral if indicated. The medical assessment consisted of examinations performed by a geriatrician, a geriatric nurse and a rehabilitation physician at the hospital. ${ }^{17}$ The assessment included a comprehensive general examination and a detailed assessment of vision, sense of hearing, locomotor apparatus, feet and footwear, peripheral nervous system, mobility, balance, anthropometry, cognition, affect, blood test if indicated and medication use. On completion of the medical assessment, the geriatrician evaluated the results and sent a written summary to the participant's general practitioner (GP). This letter included recommendations and/or referrals to relevant services, if necessary. The participants were advised to contact their GP to be informed of the results of the medical assessment and the recommendations and/or referrals to other services ensuing from it.

The occupational-therapy assessment was performed by an occupational therapist at the participant's home and comprised a functional and environmental assessment. ${ }^{17}$ On completion of this assessment, recommendations with regard to behavioural change, functional needs and safety within the home environment were immediately given to the patient. Recommendations and referrals concerning technical aids and adaptations or additional support to be provided by social and community services were implemented in accordance with the procedures prevailing in regular care. The participants received a letter with the recommendations and/or referrals, by way of reminder. A copy was sent to the participants' GPs, to inform them of the results of the assessment. 


\section{Study population}

The study population of this process evaluation can be divided into two groups:

1. All 166 participants allocated to the intervention group (referred to below as participants).

2. The medical and paramedical practitioners who performed the medical and occupational-therapy assessments (one geriatrician, three geriatric nurses, two rehabilitation physicians and two occupational therapists) (referred to below as practitioners). ${ }^{17}$

\section{Data collection}

Table 1 shows the aspects of the intervention process that were assessed and the methods used. Data were collected from participants by means of selfadministered questionnaires and structured interviews by telephone. Independent assistants asked the participants to fill out a questionnaire immediately after the medical assessment in order to assess their opinion about this assessment. For practical reasons and to avoid social desirable answers, the participants did not receive a questionnaire from the occupational therapist immediately after the occupational-therapy assessment. In order to assess the participants' opinion about the occupational-therapy assessment, detailed questions about this assessment were embedded in the structured telephone interviews which took place about six months after the recommendations and referrals had been sent to the GPs. These telephone interviews also comprised questions assessing participants' compliance with the referrals and recommendations and their overall opinion about the programme.

We used structured recording forms, structured face-to-face interviews and a plenary group discussion to collect data from the practitioners regarding the performance according to protocol, the nature of the recommendations and referrals, the compliance of the participants with the referrals and recommendations, and their opinion about the programme. The recording forms were filled out by the practitioners during or immediately after the assessments. The structured face-to-face interviews with the practitioners were scheduled immediately after all participants had undergone the assessments, and the plenary group discussion with the practitioners and the research team was carried out six months after all participants had undergone the assessments. 
Table 1 Outcome measures and measurement instruments of the process evaluation

\begin{tabular}{|c|c|c|c|c|c|c|}
\hline & \multicolumn{6}{|c|}{ Events in chronological order $\rightarrow$} \\
\hline & $\mathrm{R}$ & Q & $\mathrm{FI}$ & $\mathrm{L}$ & $\mathrm{T}$ & $\mathrm{PD}$ \\
\hline \multicolumn{7}{|l|}{ Performance of programme according to protocol } \\
\hline Deviations from protocol & $\mathrm{x}$ & & $\mathrm{x}$ & & & $\mathrm{x}$ \\
\hline Timing and duration of the assessments & $\mathrm{x}$ & $\mathrm{x}$ & $\mathrm{x}$ & & & $\mathrm{x}$ \\
\hline Nature of recommendations and referrals from assessments & & & & $\mathrm{x}$ & & \\
\hline \multicolumn{7}{|l|}{ Participants' compliance with referrals and recommendations } \\
\hline Self-reported compliance with contacting GP & & & & & $\mathrm{x}$ & \\
\hline $\begin{array}{l}\text { Self-reported compliance with referrals and recommenda- } \\
\text { tions resulting from the medical assessment }\end{array}$ & & & & & $\mathrm{x}$ & \\
\hline $\begin{array}{l}\text { Self-reported compliance with recommendations resulting } \\
\text { from the occupational-therapy assessment }\end{array}$ & & & & & $\mathrm{x}$ & \\
\hline \multicolumn{7}{|l|}{ Opinion about the programme } \\
\hline Benefit and satisfaction experienced by the participants & & $x$ & & & $\mathrm{x}$ & \\
\hline Practicability of the recording forms & & & $x$ & & & \\
\hline Acceptability of the programme to participants & & & $x$ & & & \\
\hline Recommendations (for implementation) & & & $\mathrm{x}$ & & & $\mathrm{x}$ \\
\hline
\end{tabular}

$\mathrm{R}=$ Structured recording forms for the practitioners regarding the medical and occupationaltherapy assessments; $\mathrm{Q}=$ Self-administered questionnaires for all participants who underwent the medical assessment; $\mathrm{FI}=$ Structured individual face-to-face interviews with the practitioners; $\mathrm{L}=$ Letters written by the geriatrician and occupational therapists to GPs, listing recommendations and/or referrals; $\mathrm{T}=$ Structured interviews by telephone with the participants who underwent the medical and/or the occupational-therapy assessment, about 6 months after the recommendations ensuing from the assessment(s) had been sent to the GP; PD = Plenary group discussion with the practitioners and the research team.

\section{Data analysis}

Quantitative data (e.g. duration of the assessments, perceived benefit) were analyzed by means of descriptive statistics. Qualitative data (i.e. answers to open questions in the self-administered questionnaires, individual interviews and the plenary group discussion) were classified into categories, based on the content of the answers given.

\section{Ethical considerations}

The Medical Ethics Committee of Maastricht University and the University Hospital Maastricht approved this process evaluation, being a part of the randomized controlled trial. ${ }^{17}$ 


\section{RESULTS}

\section{Attendance and response rate}

The flow of participants through the process evaluation is shown in figure 1. Of the 166 persons allocated to the intervention group, 28 (17\%) did not undergo any assessment because they withdrew from the study before the start of the assessments $(n=27)$ or had a problem with scheduling the assessments $(n=1)$. A total of 138 participants underwent at least one of the two assessments: 120 underwent both assessments, ten only the medical assessment and eight only the occupational-therapy assessment. Reasons for undergoing only one assessment were personal circumstances $(n=14)$ and withdrawal from the study before the occupational-therapy assessment was scheduled $(n=4)$. None of these reasons were related to the programme. All 130 participants who underwent the medical assessment received a selfadministered evaluation questionnaire immediately after the medical assessment. The response to this questionnaire was $100 \%$. Of the 138 participants who underwent at least one assessment, thirteen withdrew from the study after completing the medical and/or occupational-therapy assessment. The remaining 125 participants were contacted for a structured interview by phone, about six months after the results of the assessments had been sent to the GPs. Two persons could not be contacted, resulting in a response of $98 \%$. Of these 123 participants, 116 had undergone the medical assessment and 117 had undergone the occupational-therapy assessment.

The practitioners filled in recording forms during the assessments for all 130 participants who underwent the medical assessment and for all 128 participants who underwent the occupational-therapy assessment. All but one practitioner (an occupational therapist) took part in the structured face-toface interviews immediately after the implementation period of the programme. In addition, the practitioners, except one geriatric nurse and one rehabilitation physician, participated in the plenary group discussion six months after the last assessments. 


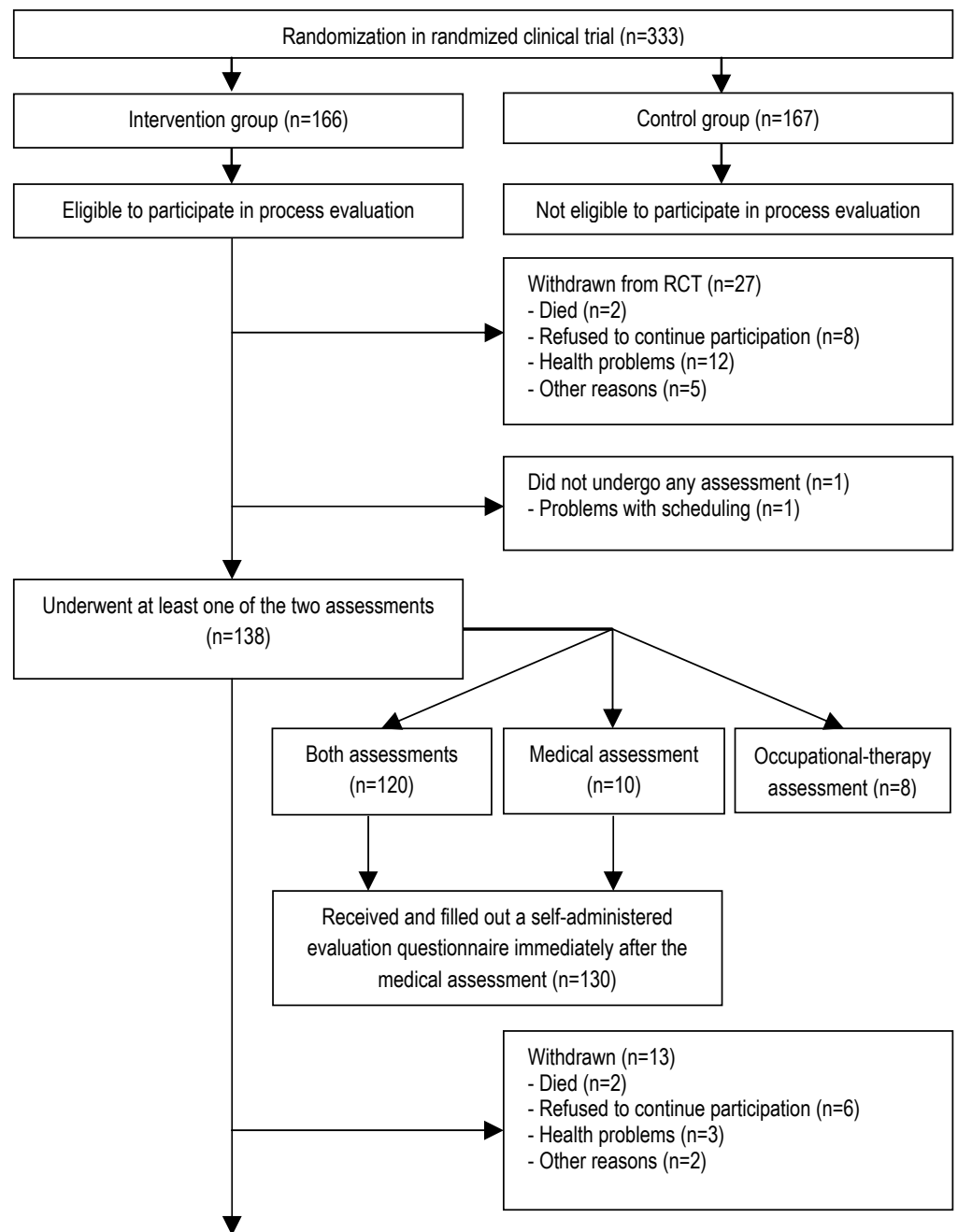

Contacted for a structured interview by telephone to evaluate the programme $(n=125)$

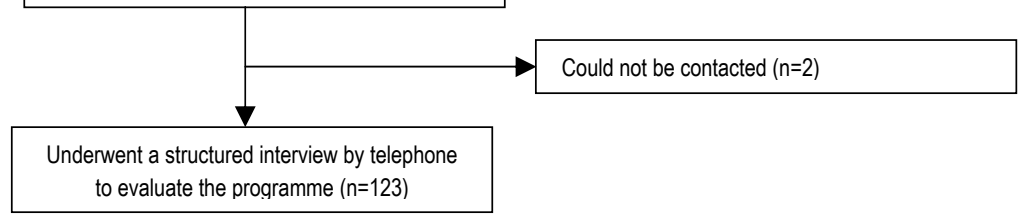

\section{Figure 1 Flow chart of participants}




\section{Performance of programme according to protocol}

\section{Protocol deviations}

The recording forms filled in by the practitioners showed that $97 \%$ of the protocol items were carried out according to protocol. Analyzing the recording forms revealed only one minor protocol deviation. During the medical assessment blood pressure was not measured in the erect position (in stead of measuring both sitting and in erect position) in 28 of the 130 participants $(22 \%)$. The information obtained from the forms was in agreement with the information gathered during the face-to-face interviews and the plenary group discussion.

Duration of the assessments, time between baseline measurement and sending the letters with recommendations

The geriatrician, the geriatric nurses and the rehabilitation physicians reported that it took 60 to 90 minutes to perform the medical assessment. The mean amount of time the geriatrician spent processing the referrals and recommendations to the GPs was estimated to be 15 minutes. The mean duration of each occupational-therapy assessment was 55 minutes and the mean time spent on processing a recording form was 21 minutes. The reported time needed for the medical and occupational-therapy assessments was in agreement with the protocol. The period between baseline measurement and sending letters to the GPs with recommendations was on average 3.5 months.

\section{Nature of the recommendations and/or referrals}

Referrals and recommendations resulting from the assessments

Table 2 shows the nature of the referrals and recommendations ensuing from the medical and occupational-therapy assessments. The referrals and recommendations made by the geriatrician comprised referrals to other specialists or therapists and recommendations concerning measures such as change of medication and orthopaedic footwear. The recommendations made by the occupational therapists can be subdivided into four categories: (1) adaptations to the home environment (e.g. installing hand rails, shower chair, raised toilet); (2) behavioural change (e.g. adapting speed of working, using antiskid mats, removing loose rugs, using hand rails); (3) health services (e.g. intake for assistive living, intake for a home for the elderly, GP consultation); and (4) assistive devices (e.g. walking device, lift chair). 
Table 2 Referrals and recommendations resulting from the medical and occupational-therapy assessments

\begin{tabular}{lc}
\hline & $\begin{array}{c}\text { Number of R/R resulting } \\
\text { from assessments }\end{array}$ \\
\hline Referrals from Medical assessments ( $n=130)$ & 8 \\
Cardiologist & 8 \\
Osteoporosis examination & 25 \\
Orthopaedic shoemaker & 1 \\
Orthopaedic instrument maker & 4 \\
Physiotherapist & 4 \\
Other referrals & 50 \\
Total & \\
& \\
Recommendations from medical assessments (n=130) & 7 \\
Adjust medication & 3 \\
Adjust footwear & 8 \\
Further examination & 2 \\
Vitamin B supplementation & 5 \\
Other recommendations & 25 \\
Total & \\
Recommendations from occupational-therapy assessments ( $n=128)$ & \\
Adaptations to the home environment & 124 \\
Behavioural change & 279 \\
Health services & 6 \\
Assistive devices & 11 \\
Total & 420 \\
\hline
\end{tabular}

$* \mathrm{R} / \mathrm{R}=$ referral $/$ recommendation

As reported by the geriatrician, the medical assessments resulted in 50 referrals and 25 recommendations for the 130 participants, which is on average 0.58 referrals/recommendations per participant. Forty-three percent of the participants $(n=56)$ received at least one referral or recommendation, and $57 \%(n=74)$ received no referral or recommendation.

As reported by the occupational therapists, 128 participants received a total of 456 recommendations (3.56 per participant) during the occupational-therapy assessments. For $91 \%$ of the participants $(n=117)$, the occupational-therapy assessment resulted in at least one referral or recommendation. For $9 \%$ $(n=11)$, it did not result in any referral or recommendation.

Overall, of the 138 participants who underwent at least one of the two assessments, 123 participants (89\%) received at least one recommendation or referral. 


\section{Participants' compliance}

\section{Contact with GP}

Of the 123 persons interviewed by telephone, 7 had not undergone a medical assessment and could therefore not answer the question whether they had contacted their GP. Of the remaining 116 participants, about half $(n=61)$ had contacted their GP to ask for the outcomes of the medical assessment, $45 \%$ $(n=52)$ had not contacted their GP and 3 persons (2\%) did not answer this question. Reasons for not contacting the GP were: forgotten $(n=28)$; not being aware of the possibility to contact the GP $(n=13)$; still intending to contact the GP $(n=6)$; not considering it necessary to contact the GP $(n=4)$ and death $(n=1)$.

\section{Self-reported compliance with recommendations and referrals}

Figure 2 reports on the net implementation of the referrals and recommendations ensuing from the medical assessments. For 14 of the participants who contacted their GP $(n=30)$, the medical assessment resulted in 28 referrals and 14 recommendations. After the implementation period of the programme, these 14 participants reported that 8 recommendations and 10 referrals had actually reached them through the GP and had been implemented. For 20 participants who did not contact their GP, the medical assessment resulted in 16 referrals and 8 recommendations. Because these participants did not contact their GPs, these referrals and recommendations did not reach the participants. However, 7 participants complied with the referral to an orthopaedic shoemaker even though none of them had contacted their GP, because the referral was made directly by the rehabilitation physician during the medical assessment.

Figure 3 reports on the net implementation of the recommendations ensuing from the occupational-therapy assessments. A total of 108 participants received 420 recommendations. At the end the implementation period of the programme, 95 of these 108 participants reported that they had received and complied with 249 recommendations.

Table 3 shows the results on the nature of the referrals and recommendations ensuing from the medical assessment for those participants who called their GP and the participants' self-reported compliance. Overall, the participants who called their GP complied with 18 of the 24 referrals and recommendations ensuing from the medical assessment, a compliance of $75 \%$. 
Table 4 shows the results on the nature of the recommendations ensuing from the occupational-therapy assessment and the participants' self-reported compliance. Overall, the participants reported having complied with $59 \%$ of the recommendations they had received from the occupational-therapy assessment.

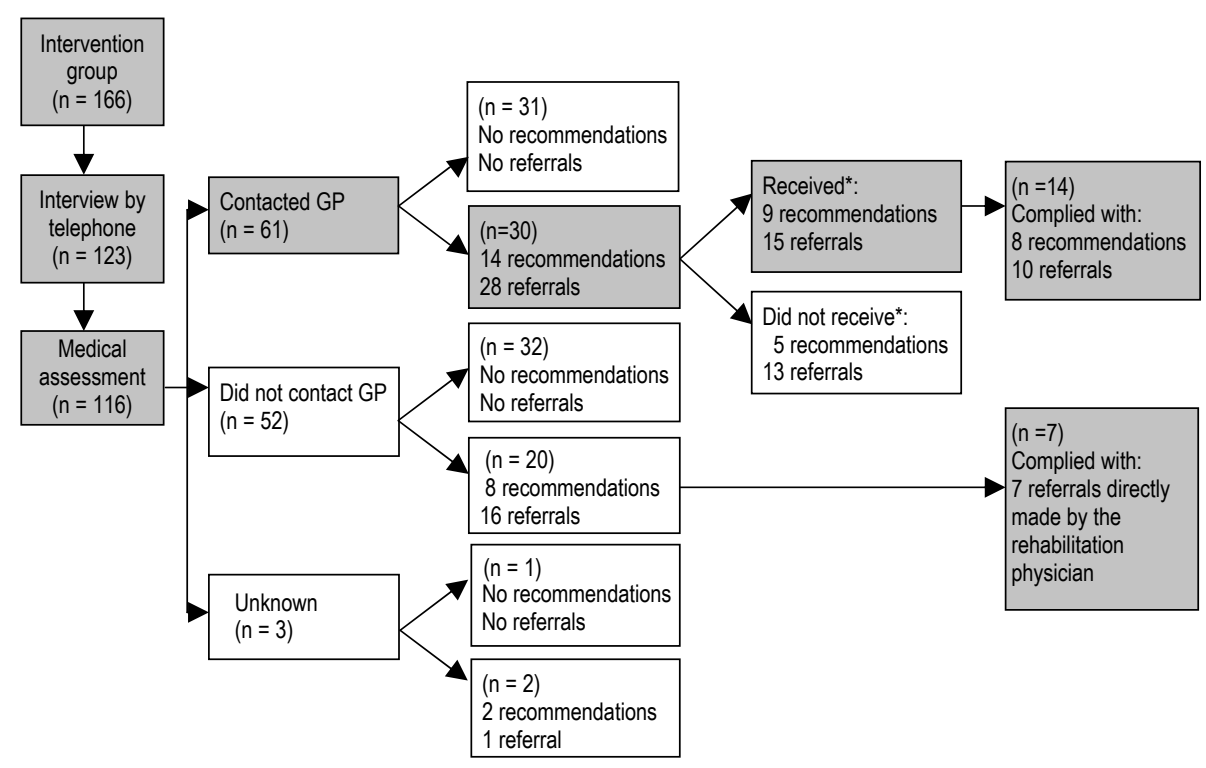

Figure 2 Net implementation of the referrals and recommendations ensuing from the medical assessment

* no numbers of participants are presented due to overlap between categories (some participants did only receive part of the referrals and/or recommendations).

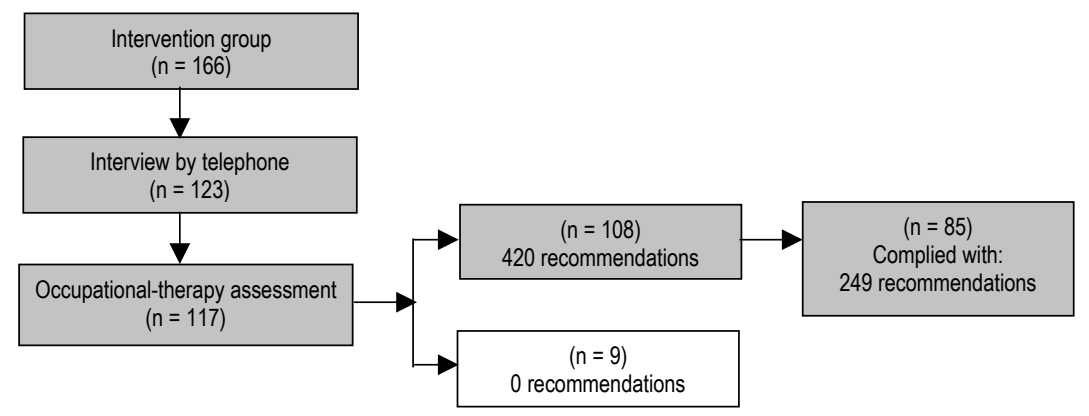

Figure 3 Net implementation of the recommendations ensuing from the occupational-therapy assessment 
Table 3 Referrals and recommendations ensuing from the medical assessment for those participants who called their GP, and participant's self-reported compliance

\begin{tabular}{lccc}
\hline & $\begin{array}{c}\mathrm{R} / \mathrm{R}^{*} \text { resulting from } \\
\text { medical assessment }\end{array}$ & $\begin{array}{c}\mathrm{R} / \mathrm{R}^{*} \text { received } \\
\text { from GP }\end{array}$ & $\begin{array}{c}\text { Self-reported } \\
\text { compliance }\end{array}$ \\
\hline Referrals & 5 & 3 & 3 \\
Cardiologist & 7 & 2 & 1 \\
Osteoporosis examination & 11 & 9 & 6 \\
Orthopaedic shoemaker & 1 & 0 & - \\
Orthopaedic instrument maker & 2 & 1 & 0 \\
Physiotherapist & 2 & 0 & - \\
Other referrals & 28 & 15 & 10 \\
Total referrals & & & 3 \\
& 3 & 3 & - \\
Recommendations & 3 & 2 & 1 \\
Adjust medication & 3 & 0 & 3 \\
Adjust footwear & 1 & 3 & 8 \\
Further examination & 4 & 9 & 18 (75\%) \\
Vitamin B supplementation & 14 & & \\
Other recommendations & & & \\
Total recommendations & 42 & & \\
Total referrals and recommenda- & & & \\
tions from medical assessment & & & \\
\hline
\end{tabular}

$* \mathrm{R} / \mathrm{R}=$ referral $/$ recommendation

Table 4 Recommendations ensuing from the occupational-therapy assessment and self-reported compliance with these recommendations

\begin{tabular}{lcc}
\hline & $\mathrm{R}^{*}$ made to participant & Self-reported compliance \\
\hline Recommendations & 124 & 68 \\
Adaptations to the home environment & 279 & 174 \\
Behavioural change & 6 & 3 \\
Health services & 11 & 4 \\
Assistive devices & & \\
Total & 420 & $249(59 \%)$ \\
\hline
\end{tabular}

$* \mathrm{R}=$ recommendation

\section{Participants' and practitioners' opinions about the programme}

\section{Participants' opinions about the programme}

During the telephone interviews, a majority of the participants reported that they had benefited from the assessments. This percentage was $82 \%$ for the medical assessments and $80 \%$ for the occupational-therapy assessments. Overall, $84 \%$ of the participants reported that they had perceived at least some benefit from the programme as a whole. Besides the perceived benefit, the participants were also asked whether they were satisfied with the medical 
and occupational-therapy assessments. Almost all participants were satisfied, viz. $97 \%$ and $99 \%$ for the medical and occupational-therapy assessments, respectively (ranging from somewhat satisfied to very satisfied).

\section{Practitioners' opinions}

The practitioners were asked to give their opinion about whether the participants had benefited from the programme. They judged that most participants had benefited, particularly those who received recommendations for footwear, adaptations to the home environment, or assistive devices. In addition, they thought that in most cases the participants were satisfied with the programme. Although the practitioners were optimistic about the programme benefits, they reported that in their opinion a considerable proportion of the participants, i.e. those with only minor health problems, should not have been included in the trial. The practitioners considered it unlikely that these persons would benefit much from the programme.

The practitioners judged the programme to be feasible and considered all aspects included in the assessments relevant. They considered the recording forms to be easy to work with, although some aspects could be improved, such as the structure and layout of the forms. They also mentioned two aspects that should be added to the programme protocol: a pre-printed list of medications that increase the risk of falling and an instrument to assess fear of falling.

The practitioners were also positive about their own role in the programme. However, they mentioned that there should be more interdisciplinary consultation and communication between the practitioners to agree on referrals and recommendations. Moreover, both assessments should be more closely tailored to the needs of individual patients and more assessments and training should be done in the home environment. To further optimize the programme, the practitioners recommended redistributing some of the assessment tasks between them, and to do some examinations more thoroughly.

\section{CONCLUSION \& DISCUSSION}

Overall, the programme turned out to be acceptable and feasible for both practitioners and participants. The results of our study show that the programme was largely performed according to protocol. The medical and occupational-therapy assessments led to an average of 3.85 recommendations 
and/or referrals per participant. However, the number of referrals and recommendations ensuing from the medical assessments was relatively small (on average 0.58) compared to the recommendations ensuing from the occupational-therapy assessments (on average 3.56). Participants' self-reported compliance with the advice to contact their GP to be informed of the recommendations and/or referrals from the medical assessment was low to moderate $(53 \%)$. Participants who were informed by their GP of the referrals and recommendations reported reasonable to good compliance $(75 \%)$ with these referrals and recommendations. Participants' self-reported compliance with the recommendations they received from the occupational therapists was moderate $(59 \%)$. Both participants and practitioners judged the programme to be feasible. A large majority of participants reported that they had benefited from the programme.

This process evaluation has provided insight into process-related factors that may explain the lack of effectiveness of our programme. The main processrelated factors that may be responsible for the lack of effectiveness are the relatively low numbers of referrals and recommendations ensuing from the medical assessments and participants' poor compliance with the suggestion to contact their GP to be informed of the recommendations and/or referrals resulting from the medical assessment.

The limited number of referrals and recommendations ensuing from the medical assessments may indicate that our study population possibly already received sufficient medical care and/or were relatively healthy and not at high risk for falls. Comparison of our population with the population of Close et al. ${ }^{16}$ however revealed that the number of recurrent fallers in our control group was comparable to the control group of Close et al. and other studies. $^{5,16,18,19}$

There are various possible explanations for the participants' low compliance with contacting their GP. Participants reported that the most important reasons for not contacting their GP were forgetting to do so, not thinking it useful, and not being aware of the possibility. These reasons may be related to the relatively long period between randomization and the moment the GPs were informed of the results of the assessments (on average 3.5 months). Recommending the participants to contact their GP and sending a subsequent reminder to all participants was apparently not sufficient to stimulate the participants to contact their GP. For our programme, this implies that it is not efficient to let the GPs act as intermediaries between the practitioners doing 
the assessments and the participants. However, our reason for incorporating the GPs was that we wanted to make the programme fit in easily with regular healthcare. In the Netherlands, referrals to medical specialists are implemented through a patient's GP. ${ }^{20}$ In addition, GPs are familiar with the health status of their patients and can therefore act as supervisors to provide the best possible care. With hindsight, including GPs in the procedure seems to be an inefficient option, and is likely to have contributed to the lack of effectiveness of our trial. In the British version of the programme, Close et al. referred their patients directly to other services or a day hospital for further investigation, assessment or follow-up ${ }^{16}$ However, it is unclear whether Close's programme would be feasible and effective when implemented in regular British care.

The present study had some possible limitations. First, participants and practitioners may have given socially desirable answers. We tried to avoid this tendency among participants by gathering data anonymously and by informing them that their answers would not affect their future use of healthcare services. Among practitioners, we tried to avoid social desirable answering by stressing that their comments and recommendations would only be used to improve the programme and not to judge their professionalism. A second limitation of this study is that we did not collect data directly from the GPs. We may have missed relevant data concerning the role of the GPs in the programme, e.g. whether the GP agreed with the suggested referrals and recommendations, and whether the participants actually called them.

\section{RECOMMENDATIONS}

Based on the results of this process evaluation and the lack of effectiveness of our programme we do not recommend implementing the programme in its present form in regular care. We recommend two major adjustments to the programme. Firstly, we recommend to screen the potential participants of the programme on their fall risk by a routinely performed short fall risk screening among patients who attend the A\&E department because of a fall. ${ }^{21-26}$ Hence it should be possible to discriminate between a low to moderate risk group and a high group among community-dwelling fallers who attending the $A \& E$ department. Focusing on fallers with a substantially increased risk of recurrent falls may improve the efficiency of the programme. Secondly, we aim to increase the efficiency of the programme by drastically decreasing the time between the patient attending the $A \& E$ department and the implementation 
of the fall prevention measures. We therefore recommend to perform the medical assessment preferably within two weeks after attending the $A \& E$ department for those directly discharged home, and around discharge for those admitted to hospital after the fall. Furthermore, the occupationaltherapy assessment should be performed preferably within two weeks after the patient is being discharged home. To further increase the efficiency, the geriatrician who performs the medical assessment should be permitted to refer patients directly to relevant services in stead of having the GP implement the referrals. The geriatrician and occupational therapist should send the GP a comprehensive report on the outcomes of the assessments and the actions already taken. This would allow the GPs to continue and coordinate the fall prevention measures initiated or implemented by the geriatrician and occupational therapist. A follow-up consultation with the geriatrician and occupational therapist after 6 months is recommended to assess the patient's current risk profile, to increase long-term compliance with fall prevention measures, and to take additional fall prevention measures if necessary. However, whether the recommended adaptations to the programme will be realizable and feasible in Dutch healthcare should be thoroughly explored, because the proposed procedure deviates considerably from usual procedures in the Netherlands. We therefore strongly recommend that both the feasibility and (cost-) effectiveness of this adjusted programme should be studied before implementing it in Dutch regular care.

\section{ACKNOWLEDGEMENTS}

This study was funded by the Netherlands Organization for Health Research and Development (ZonMw), Committee on Health Care Efficiency Research Programme, grant number 945-02-053 (info@zonmw.nl).

\section{REFERENCES}

1. Gillespie LD, Gillespie WJ, Robertson MC, Lamb SE, Cumming RG, Rowe BH. Interventions for preventing falls in elderly people. Cochrane Database Syst Rev 2003(4):CD000340.

2. Chang JT, Morton SC, Rubenstein LZ, et al. Interventions for the prevention of falls in older adults: systematic review and meta-analysis of randomised clinical trials. BMJ 2004; 328(7441):680.

3. Kannus P, Sievanen H, Palvanen M, Jarvinen T, Parkkari J. Prevention of falls and consequent injuries in elderly people. Lancet 2005;366(9500):1885-93.

4. Blake AJ, Morgan K, Bendall MJ, et al. Falls by elderly people at home: prevalence and associated factors. Age Ageing 1988;17(6):365-72. 
5. O'Loughlin J, Robitaille Y, Bolvin J-F, Suissa S. Incidence of and risk factors for falls and injurious falls among the community-dwelling elderly. Am J Epidemiol 1993;137:342-54.

6. Tinetti ME, Speechley M, Ginter SF. Risk factors for falls among elderly persons living in the community. N Engl J Med 1988;319(26):1701-7.

7. Nevitt MC, Cummings SR, Hudes ES. Risk factors for injurious falls: a prospective study. J Gerontol 1991;46(5):M164-70.

8. Fuller GF. Falls in the elderly. Am Fam Physician 2000;61(7):2159-68, 2173-4.

9. Scaf-Klomp W, van Sonderen E, Sanderman R, Ormel J, Kempen GI. Recovery of physical function after limb injuries in independent older people living at home. Age Ageing 2001; 30(3):213-9.

10. Yardley L, Donovan-Hall M, Francis K, Todd C. Older people's views of advice about falls prevention: a qualitative study. Health Educ Res 2006;21(4):508-17.

11. Vellas BJ, Wayne SJ, Romero LJ, Baumgartner RN, P.J. G. Fear of falling and restriction of mobility in elderly fallers. Age Ageing 1997;26:189-193.

12. Bloem BR, Boers, I., Cramer, M., Westendorp, R.G.J., Gerschlager, W. Falls in the elderly: identification of risk factors. Wiener Klinische Wochenschrift 2001;113:352-62.

13. Kiel DP, O'Sullivan P, Teno JM, Mor V. Health care utilization and functional status in the aged following a fall. Med Care 1991;29(3):221-8.

14. American Geriatrics Society BGS, and American Academy of Orthopaedic Surgeons Panel on Falls Prevention. Guideline for the prevention of falls in older persons. J Am Geriatr Soc 2001; 49(5):664-72.

15. Davison J, Bond J, Dawson P, Steen IN, Kenny RA. Patients with recurrent falls attending Accident \& Emergency benefit from multifactorial intervention--a randomised controlled trial. Age Ageing 2005;34(2):162-168.

16. Close J, Ellis M, Hooper R, Glucksman E, Jackson S, Swift C. Prevention of falls in the elderly trial (PROFET): a randomised controlled trial. Lancet 1999;353(9147):93-97.

17. Hendriks MR, van Haastregt JC, Diederiks JP, Evers SM, Crebolder HF, van Eijk JT. Effectiveness and cost-effectiveness of a multidisciplinary intervention programme to prevent new falls and functional decline among elderly persons at risk: design of a replicated randomised controlled trial [ISRCTN64716113]. BMC Public Health 2005;5(1):6.

18. Hendriks MR, Bleijlevens MH, van Haastregt JC, et al. Lack of effectiveness of a multidisciplinary fall prevention programme in elderly people at risk: a randomized controlled trial [ISRCTN64716113]. Accepted for Publication: J Am Geriatr Soc 2008.

19. Tinetti ME, Baker DI, McAvay G, et al. A multifactorial intervention to reduce the risk of falling among elderly people living in the community. N Engl J Med 1994;331(13):821-7.

20. Kulu Glasgow I, Delnoij D, de Bakker D. Self-referral in a gatekeeping system: patients' reasons for skipping the general-practitioner. Health Policy 1998;45(3):221-38.

21. Stalenhoef PA, Diederiks JP, Knottnerus JA, Kester AD, Crebolder HF. A risk model for the prediction of recurrent falls in community-dwelling elderly: a prospective cohort study. J Clin Epidemiol 2002;55(11):1088-94.

22. Stel VS, Pluijm SM, Deeg DJ, Smit JH, Bouter LM, Lips P. A classification tree for predicting recurrent falling in community-dwelling older persons. J Am Geriatr Soc 2003;51(10):1356-64.

23. Pluijm SM, Smit JH, Tromp EA, et al. A risk profile for identifying community-dwelling elderly with a high risk of recurrent falling: results of a 3-year prospective study. Osteoporos Int 2006; 17(3):417-25.

24. Tromp AM, Pluijm SM, Smit JH, Deeg DJ, Bouter LM, Lips P. Fall-risk screening test: a prospective study on predictors for falls in community-dwelling elderly. J Clin Epidemiol 2001;54(8):83744. 
25. Vassallo M, Stockdale R, Sharma JC, Briggs R, Allen S. A comparative study of the use of four fall risk assessment tools on acute medical wards. J Am Geriatr Soc 2005;53(6):1034-8.

26. Kalula SZ, de Villiers L, Ross K, Ferreira M. Management of older patients presenting after a fall-an accident and emergency department audit. S Afr Med J 2006;96(8):718-21. 



\section{ECONOMIC EVALUATION}

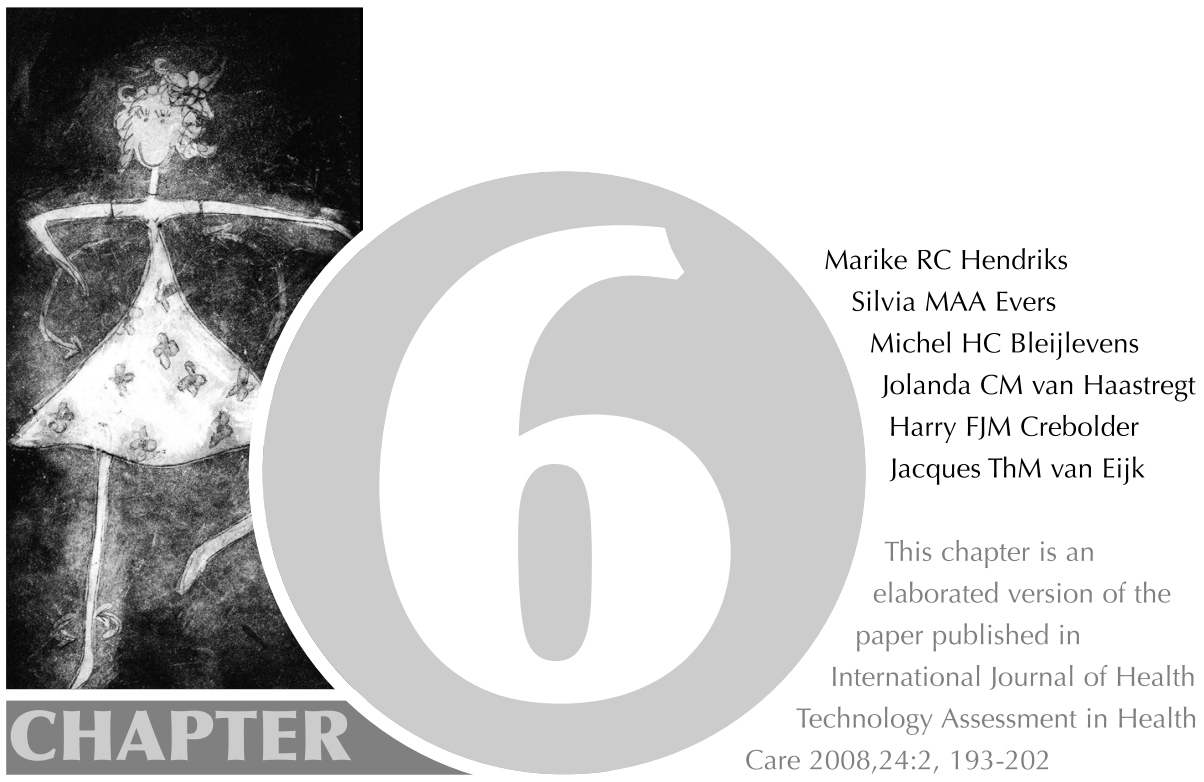




\section{ABSTRACT}

Objective. Multidisciplinary and multifactorial interventions seem to be effective in preventing falls. We aimed to assess the cost-effectiveness of a multidisciplinary fall prevention programme compared to Dutch usual healthcare in community-dwelling people aged 65 years or older who experienced a fall.

Methods. Cost-effectiveness and cost-utility analysis were performed from a societal perspective. Falls and healthcare utilization were measured continuously during twelve months. Daily functioning and quality of life were measured at baseline, after four and twelve months. Bootstrap analyses were performed to estimate uncertainty of the findings, and sensitivity analysis to assess the generalizability of assumptions made.

Results. 166 participants were randomly allocated to the experimental group and 167 to the control group. The overall response rate was $74 \%$. Healthcare and patient and family costs of both groups were comparable. Our analyses showed no effect of the intervention programme on falls, daily functioning or quality of life measures.

Conclusion. The multidisciplinary intervention programme to prevent falls was not costeffective compared to usual care in the Netherlands. Notwithstanding our findings, however, falls still have an important impact on society and individuals in terms of costs and effects. Economic evaluations studying promising interventions to prevent falls therefore remain necessary. 


\section{INTRODUCTION}

Falls are common among elderly people. About one-third of people over the age of 65 fall at least once a year and people who have sustained a fall are at greater risk of falling again., ${ }^{1,2}$ Furthermore, people who have fallen show increased morbidity and healthcare utilization, resulting in excessive healthcare costs. ${ }^{1,3-11}$ In the Netherlands, falls have been identified as a major cause of healthcare costs, and result in more than double the costs of traffic accidents. ${ }^{12}$ In terms of the diagnostic groups accounting for the highest healthcare expenditures in the Netherlands, falls rank third (accounting for $5.9 \%$ of the healthcare costs) after dementia and stroke among the oldest elderly (>85) and tenth (accounting for $2.5 \%$ of the healthcare costs) among those aged between 65 and 84 years. ${ }^{12,13}$ The largest share of the costs resulting from falls is due to hip fractures. ${ }^{14}$ Recent cost-of-illness studies confirm that falls are associated with substantial costs, ${ }^{12-19}$ and these costs are expected to rise further due to the aging population. In view of this, researchers, policymakers and practitioners in the area of fall prevention have become interested in the costs and effects of fall prevention programmes among elderly people. Currently, there is evidence for the effectiveness of multidisciplinary and multifactorial interventions that screen for health and environmental risk factors and address these risk factors in people at risk for falling. ${ }^{1,10,11}$ However, the effects of these interventions are generally small and there is some inconsistency in the results of different trials in this domain. ${ }^{1}$ Recently, some economic evaluations of fall prevention programmes have been performed, but these studies were limited in their measurement of healthcare costs, did not include patient family costs, or did not study multidisciplinary intervention programmes. ${ }^{2,20-27}$ Generally, cost-effectiveness of multidisciplinary fall prevention programmes has to be explored in greater detail. ${ }^{1}$ Therefore, we re-evaluated a British multidisciplinary intervention programme with shown effectiveness in preventing falls and functional decline, ${ }^{2}$ to evaluate its costs and effects in the Dutch healthcare setting from a broad societal perspective.

This paper reports on the economic evaluation of this multidisciplinary fall prevention programme. The evaluation aimed to assess whether this multidisciplinary intervention programme would be preferable to usual care in the Netherlands when assessed from a societal perspective in terms of costs and effects. We hypothesized that the intervention programme would lead to early detection and treatment of risk factors for falling, resulting in the 
prevention of recurrent falls ${ }^{2}$ and consequently be associated with a reduction in healthcare costs, and an improvement in the quality of life compared to usual care. We thus expected our intervention to be cost-effective.

\section{METHODS}

\section{Design and participants}

The economic evaluation was embedded in a two-group randomized controlled trial (RCT) with 12 months of follow-up. The trial design (ISRCTN 64716113) was approved by the Medical Ethics Committee of the Maastricht University and University Hospital. Details of the design have been published previously. $^{28}$

Economic evaluations compare additional costs and additional effects of an intervention programme with those of usual care. The differences in costs and effects are presented in incremental cost-effectiveness ratios (ICERs). ICERs represent the differences in mean costs between the intervention and usual care groups in the numerator and the difference in mean effects in the denominator $^{29}$ The present economic evaluation involved a combination of cost-effectiveness analysis (CEA) and cost-utility analysis (CUA). A CEA presents effects in terms of clinical outcomes (in our study falls and daily functioning), whereas a CUA presents effects in terms of quality adjusted life years (QALYs). The present economic evaluation was performed from a societal perspective, which implies that all relevant costs and outcomes were taken into account, regardless of who pays the costs and who benefits from the effects. ${ }^{30,31}$

Participants for the trial were recruited at the accident and emergency (A\&E) department and the general practitioner cooperative (GP cooperative, responsible for the out of hours A\&E care) of the Maastricht University Hospital. Participants were included if they were aged 65 years or over, and had visited the A\&E department or GP cooperative for the consequences of a fall. Participants were excluded if they were unable to speak or understand Dutch, were unable to complete questionnaires or interviews by telephone, were cognitively impaired (defined as a score of less than four on the fouritem Abbreviated Mental Test), ${ }^{32,33}$ had been admitted for more than four weeks to a hospital or other institution, and/or were permanently wheelchairdependent or bedridden. Eligible participants who signed the informed 
consent form and returned a completed baseline questionnaire were allocated to the intervention or control group by computerized alternate random allocation by an external agency. Participants allocated to the intervention group were invited to undergo a medical and occupational-therapy assessment followed by recommendations or further referral if indicated. The control group received usual care only. No restrictions were placed on cointerventions in both groups.

\section{Intervention}

The intervention was based on the interdisciplinary intervention programme developed by Close et al. ${ }^{2}$ and adapted for use in the Dutch setting. ${ }^{34}$ The intervention consisted of a medical and occupational-therapy assessment that aimed to assess and address potential risk factors for falls. The medical assessment comprised a comprehensive general examination, and detailed assessment of vision, hearing, balance, mobility, peripheral nervous system, feet, footwear, cognition, affect, and medication use. ${ }^{35}$ The medical assessment was performed by a geriatrician, a geriatric nurse and a rehabilitation physician in the hospital. On completion of the medical assessment, the geriatrician wrote a summary of the results plus recommendations and/or referrals to relevant services (if indicated) to the participant's GP, who was then expected to take action if he/she agreed with the recommendations. Participants were advised to contact their GP to be informed of the results and recommendations. After the medical assessment, an occupational therapist visited the participants at home for an assessment of daily functioning (Frenchay Activity Index, FAI) ${ }^{36}$, environmental hazards (Dutch home-safety checklist) $^{37}$ and psychological consequences of the fall (Falls Handicap Inventory, $\mathrm{FHI}){ }^{38}$ During and after the occupational-therapy assessment, recommendations with regard to behavioural change, functional needs and safety within the home environment were given to the participants. To increase compliance, a copy of the recommendations was sent to the participants and their GPs. The intervention period was scheduled to last for approximately $3 \frac{1}{2}$ months after baseline measurement.

\section{Usual Care}

Currently, no standard approach to fall risk assessment is available for fallers presenting to the A\&E department and discharged home, although such persons have a high prevalence of fall risk factors and a high risk of functional decline. ${ }^{39}$ In usual care in the Netherlands, medical risks and other risk factors 
such as environmental hazards are not systematically recorded and addressed by hospital physicians, specialists or general practitioners (GPs). Moreover, when people present to the A\&E department or the GP cooperative after a fall, the injuries are taken care of, but no systematic attention is generally paid to the specific causes of the falls.

\section{Measurements}

Participants were followed for 12 months after baseline. To ensure blinding during data collection, measurements by phone were contracted out to an independent call centre (Centre for Data and Information Management, MEMIC), whose operators have been trained to administer questionnaires and were unaware of group allocation.

\section{Cost measures}

We assessed intervention programme costs, other healthcare costs, and patient and family costs. Programme costs were based on the time the healthcare professionals spent performing the assessments, as recorded during the intervention period. The average amount of time per participant was 20 minutes for the geriatrician's consultation plus 15 minutes of administrative time per geriatrician, 20 minutes for the geriatric nurse's consultation, 20 minutes for the rehabilitation physician's consultation, and 1 hour for the home visit by the occupational therapist (OT) plus 15 minutes of administrative time for the OT. Other healthcare costs in our study related to general practitioners' consultations or home visits, inpatient and outpatient specialist care, hospital admissions, admissions to nursing homes or homes for the elderly, paramedics consultations (e.g. physiotherapy), alternative medicine, aids and assistive devices, professional home care, and prescribed and overthe-counter medication. Patient and family costs included home modifications and out-of-pocket expenses like costs of informal care and paid domestic help. Productivity losses were not assessed since they are of limited relevance in a population of mostly retired elderly persons (the age of retirement in the Netherlands being 65). All healthcare and patient and family costs were measured by means of a cost diary, ${ }^{40}$ in which participants continuously recorded volumes of healthcare utilization during the twelve-month followup period. In monthly telephone interviews, participants were asked to report the data from their cost diary for the previous month. Participants were asked whether they had received care (or devices, or services) or not. If participants answered the question on a particular topic affirmatively, they were asked to 
indicate the number of visits, hours of care or details about the specific type of healthcare utilization.

For cost valuation, we used the updated Dutch manual for cost analysis in healthcare research; for details see this manual. ${ }^{30,31}$ Briefly, we used standardized cost prices. Where no standardized cost prices were available, real costs or tariffs were used to estimate costs. In case of uncertainty, we used a conservative estimation (i.e. the lowest cost price). Costs of the intervention programme were based on standardized cost prices for care delivered by the medical and paramedical practitioners who performed the assessments. Costs of medication were calculated using prices based on Daily Defined Dosage (DDD), taken from a manual to help doctors prescribe drugs efficiently, ${ }^{41}$ Indicating mean medication use per adult per day, including claw-back (discount pharmacist are obliged to pass on to the patients). ${ }^{41,42}$ We added Value Added $\mathrm{Tax}^{42}$ plus an addition for prescription charges for prescribed medication. Prices of informal care were based on shadow prices for unpaid work (meaning a standardized cost price based on general hourly wages). Costs of transport were calculated as the mean distance per destination multiplied by standardized cost prices. Hence, costs of transportation are not presented separately, but included in the prices per subcategory.

Cost prices are presented in Euros from the baseline year 2004, and otherwise indexed to the baseline year. The consumer price index we used was $4 \%$, as suggested in the Dutch manual. ${ }^{30}$ Since the recruitment period was fourteen months and the follow-up period was relatively short (twelve months), it is unlikely that there were substantial differences in healthcare consumption and effects between participants who were included at the beginning of the recruitment period and those included towards the end. Hence, there were no reasons to discount volumes of healthcare consumption or effects.

\section{Health outcome measures}

The primary outcome measure for the cost-effectiveness analysis was the number of people sustaining a fall during one year of follow-up. Falls were recorded continuously by means of a falls calendar for twelve months after baseline. Participants were asked to report the data from their falls calendar relating to the previous month during the same monthly telephone interview in which they reported data from the cost diary. The secondary outcome measure was activities of daily living, measured by means of the Frenchay Activity Index (FAI) ${ }^{36}$ in a self-administered questionnaire that was sent to all participants at baseline and after four and twelve months of follow-up.

In the cost-utility analysis, primary outcomes were measured in terms of quality adjusted life years (QALYs). QALYs were estimated using participants' 
responses to the standard Dutch version of the EuroQol (EQ-5D) ${ }^{29,43}$ in selfadministered questionnaires at baseline and after four and twelve months. A direct value for every state of health was generated using the social tariff, ${ }^{29,44}$ which involves an algorithm for interpolating EuroQol results to population utilities. Utilities refer to preferences that individuals or society may have for a particular set of health outcomes. ${ }^{29}$ The utilities at the three measurements points (at baseline and after four and twelve months) were used to compute a QALY score by means of the area under the curve method. The area under the curve is the duration of the health state (on the x-axis) multiplied by the quality weight for the health state (on the $y$-axis), and represents the number of QALYs gained. ${ }^{29}$ In addition, specific quality of life was measured by means of the Falls Handicap Inventory $(\mathrm{FHI}){ }^{38}$ This instrument measures handicaps associated with falls. The instrument consists of 18 questions, 7 covering the emotional domain and 11 covering function/health.

\section{Analysis}

Power calculations for the present trial were based on a clinically relevant change in falls. Assuming a drop-out rate of $25 \%$, a sample of 164 patients was needed, equally distributed over the intervention and control groups. ${ }^{28}$ The analysis included those persons for whom at least $75 \%$ of the follow-up measurements of cost diary and falls calendar were available. Missing data were replaced by individual means of valid data. For the cost diary, imputation was done at the variable level before multiplying by the cost prices. For the other measures, missing data were negligible. Our primary analyses (basecase analyses) were performed according to the intention-to-treat principle, including all participants with valid data on costs and outcomes, regardless of whether they received the intervention or not. We used SPSS statistical software (version 13) and Excel (version 2003) for the analyses. While cost data are usually right-skewed and truncated to zero, decision makers are interested in the total costs of treating all patients, as well as in those persons consuming a disproportionate volume of healthcare. Therefore, despite the usual skewness in the distribution of costs, arithmetic means are generally considered the most appropriate measures to describe cost data. ${ }^{29,45}$ For this reason, arithmetic means (and standard deviations) are presented here and $\mathrm{T}$ tests were used to test for significant differences between the intervention and control groups. The robustness of the cost analyses was checked by (1000 times) bootstrapping (percentile method). ${ }^{29,46}$ Non-parametric bootstrapping is a method based on random sampling with replacement based on individual data of the participants. ${ }^{29,46}$ Uncertainty in the ICERs was also tested by means 
of bootstrapping (1000 times) based on the costs and outcomes of the participants in the trial.

One-way sensitivity analyses were performed to demonstrate the robustness of the assumptions in our base-case analyses. In a sensitivity analysis, uncertain components of the base-case analysis are varied, and results of these alternatives are compared (for example by varying costs between minimum and maximum), to assess whether these assumptions have influenced the incremental cost-effectiveness ratio (ICER). ${ }^{29,47}$ We compared our base-case CUA analyses with the following alternatives: analyses with double programme costs, complete case analysis (including all participants for whom $100 \%$ of the effect and cost data were available), per-protocol analysis (control group versus all participants who underwent both medical and occupationaltherapy assessments), and QALYs based on utilities calculated with Dutch EuroQol tariffs. ${ }^{48}$ Furthermore, we performed analyses including persons with valid data on outcomes but without cost data, by means of population mean imputation on costs.

Background characteristics were analyzed by means of T-tests or Chi-square tests. Clinical outcomes at baseline and after four and twelve months of follow-up were analyzed by means of T-tests, Chi-square tests, or in the case of a skewed distribution, Mann Whitney U-tests.

\section{RESULTS}

The study started with 333 participants, who were randomized to intervention group ( $n=166$ ) or control group $(n=167)$. At baseline, the intervention and control groups were comparable in terms of background characteristics (see table 1), quality of life and clinical outcomes (see table 3). 
Table 1 Background characteristics or outcomes measured at baseline of intervention and control groups $^{\text {a }}$

\begin{tabular}{lcc}
\hline & $\begin{array}{c}\text { Intervention group } \\
(\mathrm{n}=166)\end{array}$ & $\begin{array}{c}\text { Usual care group } \\
(\mathrm{n}=167)\end{array}$ \\
\cline { 2 - 3 } \cline { 2 - 2 } Mean $(\mathrm{sd})^{\mathrm{b}}$ age & $74.5(5.9)$ & $75.2(6.9)$ \\
Female & $111(66.9)$ & $117(70.1)$ \\
Living alone & $73(44.2)$ & $71(42.5)$ \\
$\quad$ elementary school education & $42(25.3)$ & $52(31.1)$ \\
Major injury at index fall (fracture or joint dislocation) & $66(39.8)$ & $60(35.9)$ \\
Persons who sustained more than one fall in the previous & $82(49.4)$ & $82(49.1)$ \\
year & & \\
Mean (sd) ${ }^{\mathrm{b}}$ self-reported weight & $73.4(13.7)$ & $71.8(12.3)$ \\
Mean $(\mathrm{sd})^{\mathrm{b}}$ number of illnesses & $2.8(2.0)$ & $3.2(2.5)$ \\
\hline
\end{tabular}

${ }^{a}$ Values are numbers (percentages) unless stated otherwise; ${ }^{\mathrm{b}}$ Standard deviation

Overall, $72 \%(n=120)$ of the participants in the intervention group received both the medical and occupational-therapy assessments, and $83 \%$ received at least one of the assessments. Ninety-seven percent of the items of both assessments were carried out according to protocol. After 12 months of follow-up, $25 \%(n=42)$ of the participants had dropped out of the intervention group, and $20 \%(n=33)$ out of the control group. Overall, 120 participants of the intervention group and 129 of the control group were available for cost analysis and ICER calculation. Reasons for dropout were comparable in the two groups. The main reason for dropping out of the study was health problems. Participants who withdrew from the study during the twelve-month follow-up period were on average older, had lower scores on the FAI and higher scores on the $\mathrm{FHI}$ at baseline, and reported more illnesses at baseline compared to those who completed the study. However, after twelve months of follow-up the intervention and control groups remained comparable in terms of background characteristics, except for level of education. Of the participants who remained in the study, $44(32 \%)$ in the intervention group reported only elementary school education or less, versus $26(21 \%)$ in the control group $(p=.032)$.

Table 2 shows the results of the cost analysis for the various cost categories, presented as arithmetic means and standard deviations (for details about volumes of healthcare resources consumed, please contact the first author). The mean intervention cost per participant amounted to $€ 385$. Despite these programme costs, the mean healthcare costs were somewhat lower in the intervention group. On the other hand, the intervention group showed somewhat higher mean patient and family costs. Nevertheless, the differences were not significant according to T-tests on arithmetic means or uncertainty 
intervals on bootstrapped mean differences. No significant differences between the two groups were detected in subcategories of healthcare costs or patient and family costs. Overall, mean total costs were somewhat higher in the control group $(€ 4,991)$ compared to the intervention group ( $€ 4,857)$, but this difference was not significant according to T-tests on arithmetic means or uncertainty intervals on bootstrapped mean differences.

Table 3 shows the results regarding quality of life and clinical outcome measures for the intervention and control groups. We found no significant difference between the groups as regards generic quality of life or fall-specific quality of life, nor as regards falls or daily functioning.

Since we detected differences neither in costs nor effects, it is not useful to present incremental cost-effectiveness or cost-utility ratios. Bootstrap analysis confirmed that there were no differences in effects or costs between the groups. Cost-effectiveness planes (CE-planes) visualize the results of a bootstrap analysis, with costs on the $y$-axis and effects on the $x$-axis, every dot representing an ICER computed for one bootstrap sample. Figure 1 shows the CE-plane of the CUA base-case analysis for QALYs, with only $27 \%$ of the ICERs in the dominant quadrant (representing the probability of the intervention having more effect and lower costs compared to usual care).

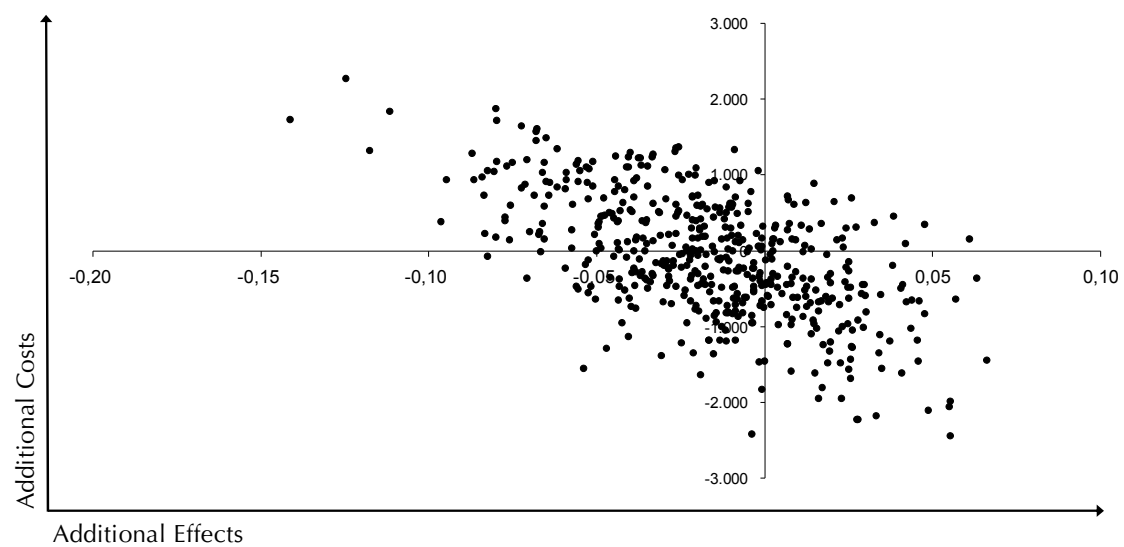

Figure 1 Cost-effectiveness plane for costs (euros) versus quality adjusted life years (QALYs) 


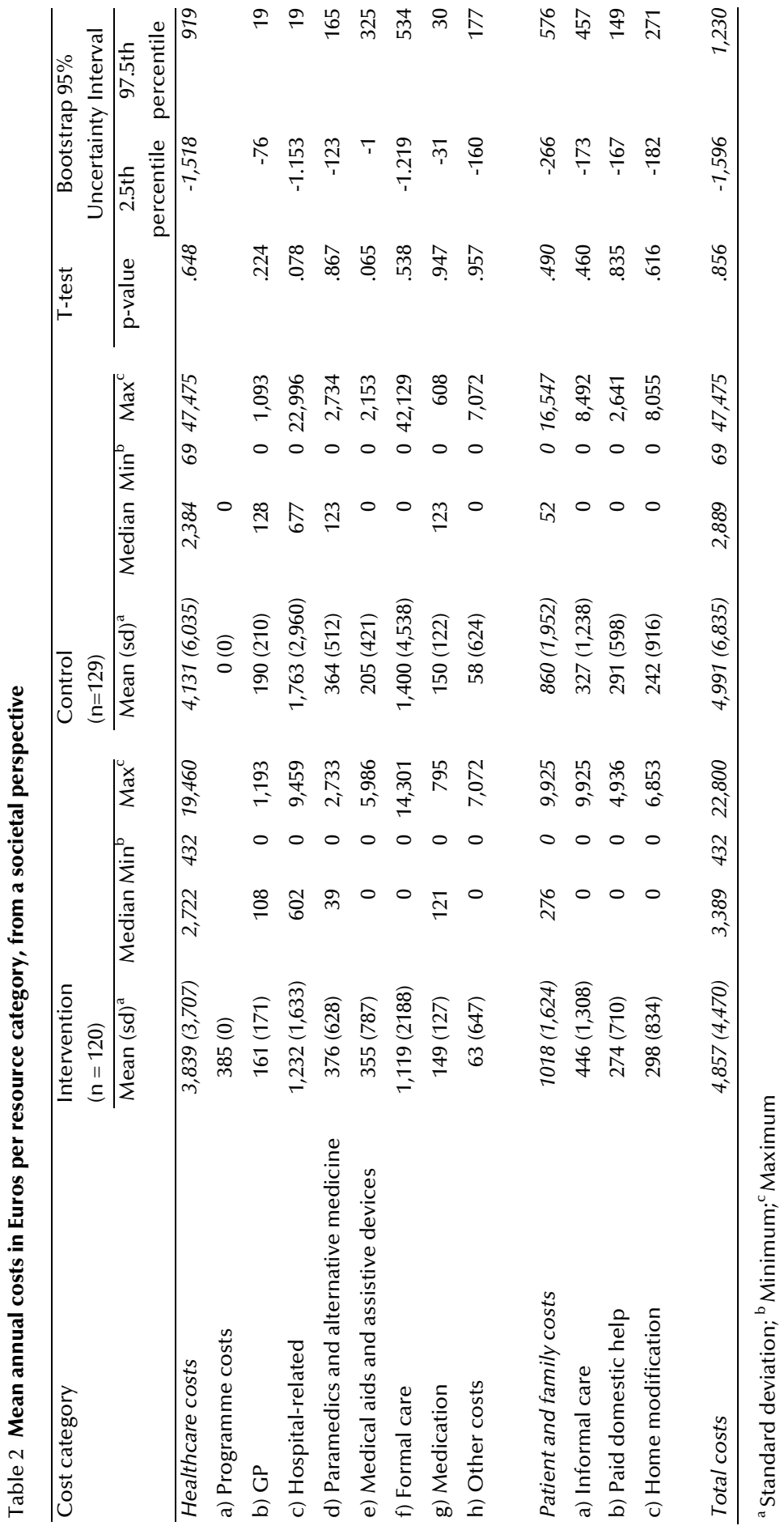




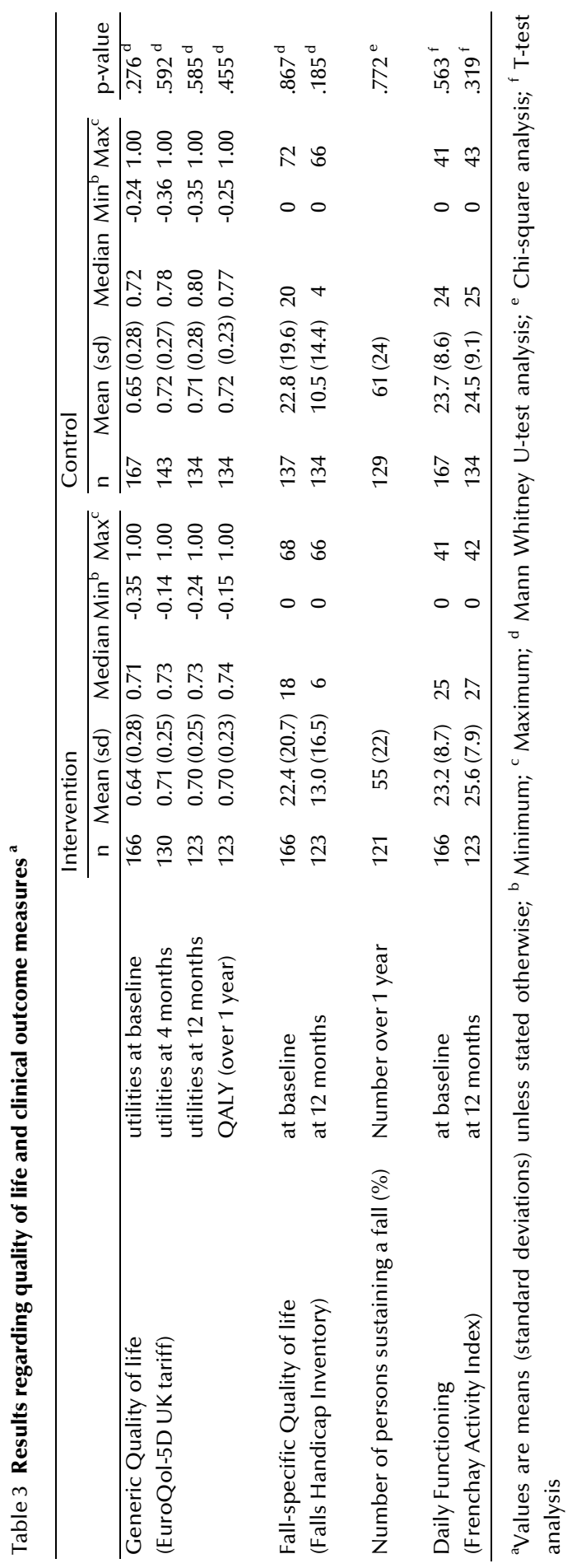


Table 4 shows the results of the sensitivity analyses to test alternatives for the assumptions we made for uncertain components in our base-case analysis. Analyses with double programme costs, per protocol analyses and complete case analyses showed no differences in cost-effectiveness ratios. The differences in costs and effects between the groups did not alter and remained statistically non-significant. Inclusion of people with valid effect data but with imputed cost data (population mean) in the analysis did not result in differences either (not tabulated).

Table 4 Results of the sensitivity analysis: Base case versus other Bootstrap analyses

\begin{tabular}{|c|c|c|c|c|c|c|c|c|}
\hline \multirow{3}{*}{ Base case ${ }^{a}$} & \multicolumn{2}{|l|}{ Participants } & \multirow[t]{2}{*}{ ICER } & \multicolumn{5}{|c|}{ Distribution on CE plane } \\
\hline & \multicolumn{2}{|c|}{ Control Intervention } & & \multirow[t]{2}{*}{$\mathrm{NE}^{\mathrm{f}} \%$} & $\begin{array}{l}\mathrm{NW}^{\mathrm{g}} \\
\text { (inferior)\% }\end{array}$ & \multicolumn{2}{|c|}{$\begin{array}{l}\mathrm{SW} \\
\mathrm{h}_{\%}\end{array}$} & $\begin{array}{l}\mathrm{SE}^{\mathrm{i}} \\
(\text { dominant }) \%\end{array}$ \\
\hline & & & & & & & & \\
\hline QALY $(U K)^{b}$ & 128 & 120 & 9,293 & 6 & 3 & 7 & 31 & 27 \\
\hline Falls & 129 & 120 & $-5,871$ & 23 & 3 & 2 & 16 & 38 \\
\hline \multicolumn{9}{|l|}{ Sensitivity analysis } \\
\hline \multicolumn{9}{|l|}{ QALY (UK) ${ }^{b}$} \\
\hline Double programme cost & 128 & 120 & 17,450 & 13 & 5 & 1 & 18 & 18 \\
\hline Complete case & 95 & 95 & 36,3794 & 7 & 3 & 2 & 33 & 29 \\
\hline Per protocol & 128 & 108 & 4,546 & 6 & 4 & 10 & 34 & 20 \\
\hline \multicolumn{9}{|l|}{ Other outcome measures } \\
\hline $\operatorname{QALY}(\mathrm{NL})^{\mathrm{c}}$ & 128 & 120 & 9,790 & 6 & 3 & 9 & 29 & 26 \\
\hline 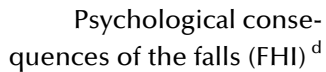 & 128 & 120 & 67 & 3 & 4 & 1 & 42 & 14 \\
\hline Functioning $(\mathrm{FAI})^{\mathrm{C}}$ & 128 & 120 & -129 & 32 & 1 & 2 & 05 & 51 \\
\hline
\end{tabular}

${ }^{\mathrm{a}}$ Base case means 1 year Follow-up, Intention to treat, 1000x Bootstrap; ${ }^{\mathrm{b}}$ QALYs based on utilities calculated with United Kingdom EuroQol tariffs; ${ }^{\mathrm{C}}$ QALYs based on utilities calculated with Dutch EuroQol tariffs; ${ }^{\mathrm{d}}$ Falls Handicap Inventory; ${ }^{\mathrm{e}}$ Frenchay Activity Index; ${ }^{\mathrm{f}}$ North East quadrant; ${ }^{\mathrm{g}}$ North

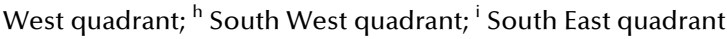

\section{DISCUSSION}

This study showed that the multidisciplinary intervention programme to prevent falls was not cost-effective compared to usual care in the Netherlands. Overall, we can conclude that healthcare utilization in the intervention and control groups was comparable. Our analyses showed no effect of the intervention programme on falls, daily functioning or quality of life measures. Also extensive effect analyses of the RCT showed no significant differences in various other outcome measures (i.e. recurrent falls, injurious falls, time to the first fall, recuperation from the fall, health complaints, perceived health, 
fear of falling, activity avoidance, mental health, ADL and IADL disability, social participation). ${ }^{35}$ Sensitivity analysis did not change our conclusions about cost-effectiveness, showing that the assumptions made in our basecase analysis were robust. Per-protocol analyses and complete case analyses did not result in differences in cost-effectiveness either.

There were no indications of selection bias in the present study. Randomization resulted in comparable intervention and control groups, and drop-outs did not alter this comparability. Most of the participants in the intervention group and none of the control group underwent the medical and occupational-therapy assessment. On the whole, we used internationally accepted and validated measurement instruments or scales. The response rate to the measurements was good.

Three limitations of the study have to be discussed. The first limitation is that the duration of follow-up was only one year. Although most studies on fall prevention programmes have a one-year follow-up period, it seems reasonable to suppose that a longer follow-up would be better able to detect differences in healthcare consumption. Since present expensive investments (e.g. home adaptations) may prevent or at least postpone high future expenditures (e.g. admission to a nursing home), cost differences may only be detectable after a longer period of follow-up. When planning new costeffectiveness studies to prevent falls by elderly people, we recommend extending the follow-up measurements of costs and effects to at least two years, to ensure that all consequences of the intervention can be measured. However, one should then keep in mind that a longer follow-up period may represent a burden to the participants, and that the number of drop-outs may increase over time. Another option would be to build a model to test the costeffectiveness of the intervention over a period longer than the time horizon of the trial. This can be done by means of decision-analytic or microsimulation models. ${ }^{29}$ The second limitation is that we did not correct for baseline differences in healthcare utilization. Since recruitment of participants took place after they had sustained a fall, we have no baseline information about healthcare utilization before the fall. However, because participants were randomly allocated to the intervention and control groups, we have no reason to assume differences in healthcare utilization between the groups. The third limitation is that our analyses were not restricted to costs related to falls only, but compared all healthcare costs and patient and family costs. Because falls account for only part of the total health expenditures of elderly people, differences in costs related to falls might not be observable. 
However, in view of the variety of risk factors for falls (including poor eyesight, mobility problems and neurological problems) it is hardly possible to distinguish costs related to falls from other healthcare costs.

This is the first published economic evaluation in the Netherlands to assess the costs and effects of a multidisciplinary intervention programme to prevent falls from a broad societal perspective. Although some other attempts have been made to study cost-effectiveness of fall prevention programmes, these are difficult to compare with the current study because they differ in methodology or studied other aspects of fall prevention programmes.,20-27 We reevaluated the study by Close and her colleagues in the UK. In terms of the costs associated with the intervention programme, no detailed comparison can be made with this study we re-evaluated due to limited data available. ${ }^{2}$ Close only reported that the programme was cost-neutral. ${ }^{49}$ Our study also found the intervention to be cost-neutral. It is remarkable, however, that Close $^{2}$ and her colleagues reported the intervention to be highly effective in preventing falls in the UK, while our study demonstrated no such effects. Overall, we conclude that although the intervention programme was costneutral in the Netherlands, it did not prevent falls or functional decline, and did not improve quality of life. Therefore, although the intervention did not result in higher costs for the intervention group, we do not recommend implementing the intervention programme in its present form in the Netherlands. At the same time, however, it must be said that falls do have an important impact on society as well as on individuals in terms of costs and effects, and economic evaluations of promising interventions to prevent falls therefore remain necessary.

\section{ACKNOWLEDGEMENTS}

Preliminary results of this cost-effectiveness analysis were presented at the $5^{\text {th }}$ Annual Meeting of the Gerontological Society of America, Dallas, USA November 2006. The full paper was presented at the International Health Economics Association (IHEA) Sixth World Congress: Explorations In Health Economics, Copenhagen, Denmark, Juli 2007. The study was funded by The Netherlands Organization for Health Research and Development (ZonMw), Committee Health Care Efficiency Research Programme, grant number 945-02053 (info@zonmw.nl) 


\section{REFERENCES}

1. Gillespie LD, Gillespie WJ, Robertson MC, Lamb SE, Cumming RG, Rowe BH. Interventions for preventing falls in elderly people. Cochrane Database Syst Rev 2003(4):CD000340.

2. Close J, Ellis M, Hooper R, Glucksman E, Jackson S, Swift C. Prevention of falls in the elderly trial (PROFET): a randomised controlled trial. Lancet 1999;353(9147):93-97.

3. Blake AJ, Morgan K, Bendall MJ, et al. Falls by elderly people at home: prevalence and associated factors. Age Ageing 1988;17(6):365-72.

4. Tinetti ME, Speechley M, Ginter SF. Risk factors for falls among elderly persons living in the community. N Engl J Med 1988;319(26):1701-7.

5. Nevitt MC, Cummings SR, Hudes ES. Risk factors for injurious falls: a prospective study. J Gerontol 1991;46(5):M164-70.

6. Speechley M, Tinetti M. Falls and injuries in frail and vigorous community elderly persons. J Am Geriatr Soc 1991;39(1):46-52.

7. Sattin RW, Rodriguez JG, DeVito CA, Wingo PA. Home environmental hazards and the risk of fall injury events among community-dwelling older persons. J Am Geriatr Soc 1998;46:669-76.

8. Kiel DP, O'Sullivan P, Teno JM, Mor V. Health care utilization and functional status in the aged following a fall. Med Care 1991;29(3):221-8.

9. Rizzo JA, Friedkin R, Williams CS, Nabors J, Acampora D, Tinetti ME. Health care utilization and costs in a Medicare population by fall status. Med Care 1998;36(8):1174-88.

10. Chang JT, Morton SC, Rubenstein LZ, et al. Interventions for the prevention of falls in older adults: systematic review and meta-analysis of randomised clinical trials. BMJ 2004; 328(7441):680.

11. Kannus $P$, Sievanen $H$, Palvanen $M$, Jarvinen T, Parkkari J. Prevention of falls and consequent injuries in elderly people. Lancet 2005;366(9500):1885-93.

12. Meerding WJ, Mulder S, van Beeck EF. Incidence and costs of injuries in The Netherlands. Eur J Public Health 2006;16(3):272-8.

13. Meerding WJ, Bonneux L, Polder JJ, Koopmanschap MA, van der Maas PJ. Demographic and epidemiological determinants of healthcare costs in Netherlands: cost of illness study. BMJ 1998;317(7151):111-5.

14. Polder JJ, Takken J, W.J. M, Kommer GJ, Stokx LJ. Kosten van Ziekten in Nederland. De zorgeuro ontrafeld. Bilthoven: Rijksinstituut voor Volksgezondheid en Milieu, 2002: 1-304.

15. Scuffham P, Chaplin S, Legood R. Incidence and costs of unintentional falls in older people in the United Kingdom. J Epidemiol Community Health 2003;57(9):740-4.

16. Carroll NV, Slattum PW, Cox FM. The cost of falls among the community-dwelling elderly. J Manag Care Pharm 2005;11(4):307-16.

17. Newton JL, Kyle P, Liversidge P, Robinson G, Wilton K, Reeve P. The costs of falls in the community to the North East Ambulance Service. Emerg Med J 2006;23(6):479-81.

18. Stevens JA, Corso PS, Finkelstein EA, Miller TR. The costs of fatal and non-fatal falls among older adults. Inj Prev 2006;12(5):290-5.

19. Roudsari BS, Ebel BE, Corso PS, Molinari NA, Koepsell TD. The acute medical care costs of fallrelated injuries among the U.S. older adults. Injury 2005;36(11):1316-22.

20. Rizzo JA, Baker DI, McAvay G, Tinetti ME. The cost-effectiveness of a multifactorial targeted prevention program for falls among community elderly persons. Med Care 1996;34(9):954-69.

21. Smith RD, Widiatmoko D. The cost-effectiveness of home assessment and modification to reduce falls in the elderly. Aust N Z J Public Health 1998;22(4):436-40.

22. Salkeld G, Cumming RG, O'Neill E, Thomas M, Szonyi G, Westbury C. The cost effectiveness of a home hazard reduction program to reduce falls among older persons. Aust $N$ Z J Public Health 2000;24(3):265-71. 
23. Robertson MC, Devlin N, Gardner MM, Campbell AJ. Effectiveness and economic evaluation of a nurse delivered home exercise programme to prevent falls. 1: Randomised controlled trial. BMJ 2001;322(7288):697-9.

24. Robertson MC, Devlin N, Scuffham P, Gardner MM, Buchner DM, Campbell AJ. Economic evaluation of a community based exercise programme to prevent falls. J Epidemiol Community Health 2001;55(8):600-6.

25. Robertson MC, Gardner MM, Devlin N, McGee R, Campbell AJ. Effectiveness and economic evaluation of a nurse delivered home exercise programme to prevent falls. 2: Controlled trial in multiple centres. BMJ 2001;322(7288):701-4.

26. Campbell AJ, Robertson MC, La Grow SJ, et al. Randomised controlled trial of prevention of falls in people aged $>$ or $=75$ with severe visual impairment: the VIP trial. BMJ 2005; 331(7520):817.

27. Beard J, Rowell D, Scott D, et al. Economic analysis of a community-based falls prevention program. Public Health 2006;120(8):742-51.

28. Hendriks MR, van Haastregt JC, Diederiks JP, Evers SM, Crebolder HF, van Eijk JT. Effectiveness and cost-effectiveness of a multidisciplinary intervention programme to prevent new falls and functional decline among elderly persons at risk: design of a replicated randomised controlled trial [ISRCTN64716113]. BMC Public Health 2005;5(1):6.

29. Drummond MF, Sculpher MJ, Thorrance GW, O'Brien BJ, Stoddart GL. Methods for the Economic Evaluation of Health Care Programmes. Third ed. New York: Oxford University Press, 2005.

30. Oostenbrink JB, Bouwmans CAM, Koopmanschap MA, Rutten FFH. Handleiding voor kosten onderzoek; methoden en standaard kostprijzen voor economische evaluaties in de gezondheidszorg (Geactualiseerde versie) [Dutch manual for costing: methods and standard costs for economic evaluations in healthcare]. Diemen: College voor zorgverzekeringen, 2004.

31. Oostenbrink JB, Koopmanschap MA, Rutten FF. Standardisation of costs: the Dutch Manual for Costing in economic evaluations. Pharmacoeconomics 2002;20(7):443-54.

32. Swain DG, Nightingale PG. Evaluation of a shortened version of the Abbreviated Mental Test in a series of elderly patients. Clin Rehabil 1997;11(3):243-8.

33. Swain DG, O'Brien AG, Nightingale PG. Cognitive assessment in elderly patients admitted to hospital: the relationship between the shortened version of the Abbreviated Mental Test and the Abbreviated Mental Test and Mini-Mental State Examination. Clin Rehabil 2000;14(6):608-10.

34. Hendriks MR, Bleijlevens $M H$, van Haastregt JC, et al. A multidisciplinary fall prevention program for elderly persons: a feasibility study. Geriatric Nursing: accepted for publication; available upon request 2008:000-000.

35. Hendriks MR, Bleijlevens $M H$, van Haastregt JC, et al. Effectiveness of a multidisciplinary fall prevention programme in elderly people at risk: a randomized controlled trial [ISRCTN64716113]. Provisionally accepted: available upon request 2008.

36. Schuling J, de Haan R, Limburg M, Groenier KH. The Frenchay Activities Index. Assessment of functional status in stroke patients. Stroke 1993;24(8):1173-7.

37. Stalenhoef P, Diederiks J, Knottnerus A, de Witte L, Crebolder H. How predictive is a homesafety checklist for indoor fall risk in the elderly living in the community? Eur J Gen Pract 1998; 4:114-120.

38. Rai GS, Kiniorns M, Wientjes H. Falls Handicap Inventory (FHI)--an instrument to measure handicaps associated with repeated falls. J Am Geriatr Soc 1995;43(6):723-4.

39. Russell MA, Hill KD, Blackberry I, Day LL, Dharmage SC. Falls risk and functional decline in older fallers discharged directly from emergency departments. J Gerontol A Biol Sci Med Sci 2006;61(10):1090-5. 
40. Goossens ME, Rutten van Molken MP, Vlaeyen JW, van der Linden SM. The cost diary: a method to measure direct and indirect costs in cost-effectiveness research. J Clin Epidemiol 2000;53(7):688-95.

41. Commissie Farmacotherapeutische Hulp CVZ. Farmacotherapeutisch Kompas [pharmacotherapeutic manual]. 21 ed. Amstelveen: College voor zorgverzekeringen, 2005.

42. Tinke J, Griens A. Data en Feiten 2004 [Data and facts for 2004]. Den Haag: Stichting Farmacotherapeutische Kengetallen, 2004.

43. Brooks R. Euroqol:the current state of play. Health policy 1996;37(1):53-72.

44. Dolan P. Modeling valuations for EuroQol health states. Med Care 1997;35(11):1095-108.

45. Ramsey S, Willke R, Briggs A, et al. Good research practices for cost-effectiveness analysis alongside clinical trials: the ISPOR RCT-CEA Task Force report. Value Health 2005;8(5):521-33.

46. Briggs $\mathrm{AH}$, Wonderling DE, Mooney CZ. Pulling cost-effectiveness analysis up by its bootstraps: a non-parametric approach to confidence interval estimation. Health Econ 1997;6(4):327-40.

47. Briggs A, Sculpher M, Buxton M. Uncertainty in the economic evaluation of health care technologies: the role of sensitivity analysis. Health Econ 1994;3(2):95-104.

48. Lamers LM, Stalmeier PF, McDonnell J, Krabbe PF, van Busschbach JJ. [Measuring the quality of life in economic evaluations: the Dutch EQ-5D tariff]. Ned Tijdschr Geneeskd 2005;149(28):1574-8.

49. Close JC, Hooper R, Glucksman E, Jackson SH, Swift CG. Predictors of falls in a high risk population: results from the prevention of falls in the elderly trial (PROFET). Emerg Med J 2003; 20(5):421-5. 



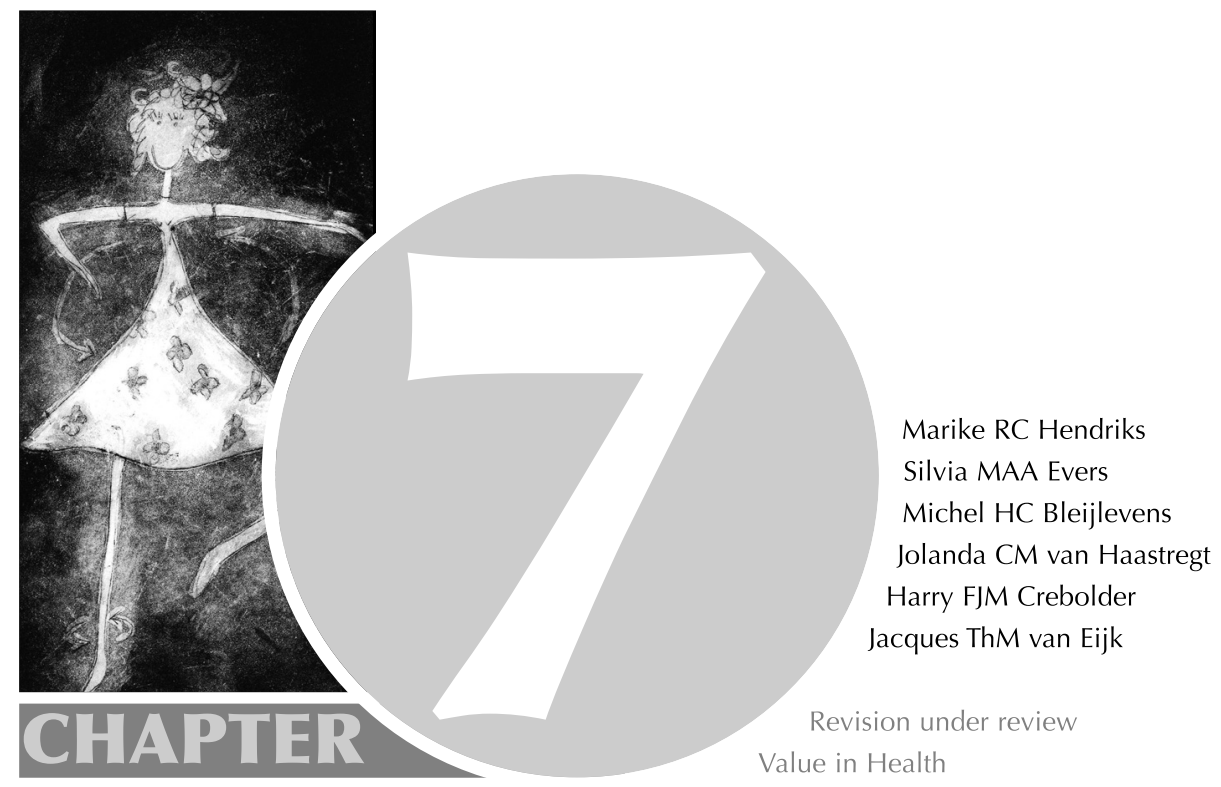




\section{ABSTRACT}

Objective. Economic evaluations are preferably performed from a societal perspective. This implies that participants report their healthcare utilization continuously during followup. Because this is a burden to participants, often resulting in missing values or withdrawal, researchers advocate collecting data discontinuously (i.e. in at least three months a year). This study aimed to compare costs of discontinuous measurement of healthcare utilization with those of continuous measurement, using several discontinuous measurement patterns and three imputation techniques: Individual Mean (IM), Last Observation Carried Forward (LOCF) and Next Observation Carried Backward (NOCB).

Methods. We used continuous healthcare utilization data from a trial with twelve months' follow-up and simulated several discontinuous measurement patterns combined with different imputation methods, to calculate simulated annual volumes and costs. Correlations and paired T-tests were used to compare simulated annual costs with actual annual costs from the continuous measurements.

Results. Analyses confirmed that discontinuous measurements using cost diaries offer good estimates of annual health expenditures, but measurement patterns and imputation methods did influence the outcomes, as the correlations differed between methods. The best estimated annual costs were obtained by random cohort measurement, using three random cohorts, ensuring that at least a third of the participants were measuring costs each month, combined with IM imputation. Discontinuous measurement of health expenditures carries a small risk of missing infrequent expensive events, which may result in underestimation of annual costs.

Conclusions. To reduce the burden on participants in future economic evaluation, we recommend calculating annual costs from discontinuous measurements in random cohorts, combined with IM imputation. 


\section{INTRODUCTION}

In the coming years, healthcare costs are expected to rise in Western countries, as a result of various developments. Demographic changes caused by the post-war baby boom will result in a growing number of elderly people and hence in increased morbidity and multi-morbidity. ${ }^{1}$ In addition, the number of new healthcare interventions is growing rapidly as a result of medicotechnological progress as well as cultural changes, such as increasing demands for healthcare at lower complaint levels. ${ }^{1}$ In face of limited financial resources, there is an obvious need to improve the efficiency of healthcare. This together with the current availability of economic evaluation and analysis techniques explains the increase in the number of economic evaluation in healthcare research. Economic evaluations use different sources to obtain information about health expenditures, for example databases of insurance companies, healthcare providers (hospitals or other healthcare institutions), or self-reports by patients. ${ }^{2-4}$

Economic evaluations should preferably be performed from a societal perspective, which means that all relevant costs and effects should be included. ${ }^{2,5}$ For costs, this implies measuring not only costs within the official healthcare sector but also out-of-pocket expenses for patients, for example overthe-counter medication, informal care, travelling expenses, and productivity losses. ${ }^{2,5}$ No single database can provide this information and the only person who can provide an overview of the total scope of healthcare utilization and other expenses is the patient. Patient information is thus an important and valuable information source for economic evaluations. ${ }^{2,6}$

Information from patients can be retrieved using various instruments, such as diaries, questionnaires, and interviews. ${ }^{2,6-8}$ The validity of self-report data may however be affected by the way the data are collected, for example by the burden imposed by the data collection instrument, the length of the recall period, and social desirability. ${ }^{3,6}$ From a societal perspective, patients should preferably report their use of resources continuously during the follow-up of the economic evaluation, which can last up to several years in some studies. We define continuous reporting as the use of any measurement technique by which researchers can gain insight into the healthcare utilization for every day during the timeframe of the study. Continuous data can be obtained prospectively, using cost-diaries, or retrospectively, using questionnaire or interviews. 
Diaries offer some advantages over questionnaires, as they ensure continuous registration, measure healthcare consumption prospectively and are more accurate. $^{2,7-9}$ Disadvantages of diaries are the relatively low compliance with their completion and a relatively high level of missing values, especially over a long follow-up period. ${ }^{6-8}$ Questionnaires have the advantage of being less time-consuming and expensive, but are retrospective, so rely on momentary recall, which can cause recall bias. ${ }^{2,6,9}$ Whatever method is used, continuous data collection imposes a burden on participants, so missing values and withdrawal are common. ${ }^{6,10-12}$ To relieve the burden on participants, data collection of healthcare utilization is often performed periodically (discontinuous data collection), instead of continuously during the entire evaluation period. $^{2,13-19}$

Previous studies revealed that measuring in at least three months during a year provides good estimates of annual costs. ${ }^{2,13}$ However, as far as we know there is no evidence about the most appropriate choice of measurement patterns. Different patterns of data collection have been used for discontinuous health expenditures measurement, ${ }^{2,13,14,18}$ for example measuring during the first and last months of a year, and one month in between (e.g. months $1,6,12)$. Some studies used intensive measurement during those periods in which higher costs were expected, such as for the duration of an injury, during recovery, or during and immediately after treatment. ${ }^{15,16}$ In a fall prevention trial studying participants after they had sustained an injurious fall, this would mean measuring during some months after the inclusion and at the end of the follow-up (e.g. months 1,2,3,12). Occasionally studies measure during random periods, in order to obtain a random sample of individual healthcare consumption. ${ }^{17}$ This method randomly assigns all subjects to, for example, three cohorts and measures all cohorts a number of times a year, with the various cohorts starting their measurements in successive months (e.g. cohort 1 measures health expenditures in months 1, 4, 7, and 10; cohort 2 in months 2, 5, 8, and 11; and cohort 3 in months 3, 6, 9, and 12).

When data are collected discontinuously using the above methods, total annual costs have to be estimated from the data for the periods for which they are available. Annual health expenditures are then estimated by interpolating (calculating a new point between two existing data points) or extrapolating (constructing new data points outside a discrete set of known data points). Although several papers have discussed ways of dealing with unavailable data caused by incomplete observations or withdrawal (missing values), ${ }^{10-12}$ less attention has been paid to ways of interpolating or extrapolating data 
that are intentionally unavailable because of discontinuous data collection. Moreover, reports on studies extrapolating data have not always clearly described how this was done. ${ }^{14,18,19}$ Traditionally, annual cost data are obtained from discontinuous data collection by multiplying data for the available periods (e.g. 3 months multiplying by 4 to obtain annual costs, which is the same as individual mean imputation, in which the missing data are filled in using the calculated average of the available periods). ${ }^{2,13}$ Other common methods to estimate annual costs are the last-observation-carried-forward (LOCF) method, in which missing values for each individual are replaced by the last observed value of that variable, or the opposite method, called nextobservation-carried-backward (NOCB). ${ }^{2,16}$

The problem with discontinuous measurements is the risk of missing important changes in between measurements, such as missing a temporary or sudden decline in health status. ${ }^{8}$ This may result in missing expensive events like hospital admissions or the purchase of expensive aids like a mobility scooter. Healthcare expenditures can be classified into four types: (1) frequent health expenditures with low costs, (2) frequent health expenditures with high costs, (3) infrequent health expenditures with low costs, (4) infrequent health expenditures with high costs. Measuring health expenditures discontinuously carries a risk of missing infrequent events, which may result in underestimation of annual costs, especially when the missed health expenditures are the expensive ones (type 4).

As regards discontinuous measurement of healthcare utilization, there is a need for additional information about the preferred measurement patterns and the preferred way to impute the unavailable data. Furthermore, it is necessary to know whether combinations of different measurement patterns and different imputation methods are sufficiently sensitive to measure various types of costs. Therefore, the present study explored which combination of discontinuous measurement patterns and imputation techniques results in the best estimate of annual costs for different cost types. 


\section{METHODS}

\section{Participants and measurements}

Data for this study were obtained from a two-group randomized controlled trial (RCT) evaluating the effectiveness and cost-effectiveness of a fall prevention programme [ISRCTN64716113]. ${ }^{20}$ Persons eligible to participate in the RCT were community-dwelling elderly people aged 65 years and over who attended the Accident and Emergency (A\&E) department of the University Hospital Maastricht (the Netherlands) for the consequences of a fall. Participants randomly allocated to the control group received usual care, while participants allocated to the intervention group received a medical and occupational-therapy assessment followed by recommendations and/or further referral if indicated. The intervention period was scheduled to last around three months from the baseline measurement, and the RCT included 12 months of follow-up. ${ }^{20}$ Resource utilization was measured prospectively by the participants by means of a validated monthly cost diary, ${ }^{2}$ in which participants continuously recorded volumes of healthcare utilization during the twelve-month follow-up period. In monthly telephone interviews, participants were asked to report the data from their cost diary for the previous month.

Analyses for the present study were restricted to those participants with complete data on resource utilization. Resource utilization in this study related to general practitioner consultations, specialist care, hospital admissions, ambulance transport, consultations with allied health professionals (e.g. physiotherapy, alternative medicine), social services, aids and assistive devices, home modifications, professional home care, informal care, and paid domestic help.

For cost valuation, we used the updated Dutch manual for cost analysis in healthcare research; for details see this manual. ${ }^{21,22}$ Briefly, we used standardized cost prices. Where no standardized cost prices were available, real costs or tariffs were used to estimate costs. Cost prices are presented in Euros from the baseline year 2004, and otherwise indexed to the baseline year. The consumer price index we used was $4 \%$, as suggested in the Dutch manual. ${ }^{21}$

\section{Design}

To explore the best estimate of various discontinuous measurement patterns combined with several imputation techniques, we simulated discontinuous 
data collection on individual level and calculated annual volumes of healthcare utilization using different imputation techniques. Subsequently, we compared these simulated annual volumes with the actual continuously measured volumes obtained over twelve months, referred to below as "true volumes". Next we multiplied annual volumes with the cost prizes to obtain annual costs, and compared the simulated annual costs with the actual costs obtained over twelve months, referred to below as "true costs".

We performed three different pattern simulations (see the overview in table 1). For the first data simulation, we calculated annual costs based on data obtained in three months during the year, namely the first, the last and one in the middle (i.e. months 1,6, and 12); we refer to this as "SIM1". Secondly, we calculated annual costs based on data obtained during the period in which higher costs were expected and data obtained at the end of the follow-up period (i.e. months 1, 2, 3, and 12), referred to as "SIM2". Thirdly, we calculated annual costs based on random periods in three sub-cohorts, with at least one-third of the participants measuring costs in each month (i.e. the first cohort measuring in months 1,4, 7, and 10; the second in months 2, 5, 8, and 11 ; and the third in months $3,6,9$, and 12); this is referred to as "SIM3".

Subsequently, we used three common imputation methods to calculate annual costs for the simulations. We did not use multiple imputation techniques and limited ourselves to single imputation methods, as these are most likely to be used in common practice. We first examined individual mean imputation (IM), in which the sum of the observations available for each individual is multiplied to obtain annual costs. This imputation method could be used in all simulations. Secondly, when possible (i.e. if the first month was available) we examined the last-observation-carried-forward (LOCF) method, in which unavailable data are imputed from the data for the previous period. Thirdly, when possible (i.e. if the last month was available) we examined the next-observation-carried-backward (NOCB) method, in which unavailable data are imputed from the data for the subsequent period. The different measurement patterns, combined with the different imputation methods, resulted in seven different simulations, which were then compared with the true data (for details see table 1 ). 
Table 1 Overview of true annual costs and seven simulations of annual costs*

\begin{tabular}{lccccccccccccc}
\hline & MONTHS & & & & & & & & & & $\begin{array}{l}\text { ANNUAL } \\
\text { COSTS }\end{array}$ \\
\hline TRUE COSTS & $\mathbf{1}$ & $\mathbf{2}$ & $\mathbf{3}$ & $\mathbf{4}$ & $\mathbf{5}$ & $\mathbf{6}$ & $\mathbf{7}$ & $\mathbf{8}$ & $\mathbf{9}$ & $\mathbf{1 0}$ & $\mathbf{1 1}$ & $\mathbf{1 2}$ & $\sum(1-12)$ \\
SIMULATIONS & & & & & & & & & & & & & \\
SIM1 IM & $\mathbf{1}$ & - & - & - & - & $\mathbf{6}$ & - & - & - & - & - & $\mathbf{1 2}$ & $(1+6+12) \times 4$ \\
SIM1 LOCF & $\mathbf{1}$ & 1 & 1 & 1 & 1 & $\mathbf{6}$ & 6 & 6 & 6 & 6 & 6 & $\mathbf{1 2}$ & $(5 \times 1)+(6 \times 6)+12$ \\
SIM1 NOCB & $\mathbf{1}$ & 6 & 6 & 6 & 6 & $\mathbf{6}$ & 12 & 12 & 12 & 12 & 12 & $\mathbf{1 2}$ & $1+(5 \times 6)+(6 \times 12)$ \\
SIM2 IM & $\mathbf{1}$ & $\mathbf{2}$ & $\mathbf{3}$ & - & - & - & - & - & - & - & - & $\mathbf{1 2}$ & $(1+2+3+12) \times 3$ \\
SIM2 LOCF & $\mathbf{1}$ & $\mathbf{2}$ & $\mathbf{3}$ & 3 & 3 & 3 & 3 & 3 & 3 & 3 & 3 & $\mathbf{1 2}$ & $1+2+3+(8 \times 3)+12$ \\
SIM2 NOCB & $\mathbf{1}$ & $\mathbf{2}$ & $\mathbf{3}$ & 12 & 12 & 12 & 12 & 12 & 12 & 12 & 12 & $\mathbf{1 2}$ & $1+2+3+(9 \times 12)$ \\
SIM3 IM Cohort I & $\mathbf{1}$ & - & - & $\mathbf{4}$ & - & - & $\mathbf{7}$ & - & - & $\mathbf{1 0}$ & - & - & \\
\multicolumn{1}{c}{ Cohort II } & - & $\mathbf{2}$ & - & - & $\mathbf{5}$ & - & - & $\mathbf{8}$ & - & - & $\mathbf{1 1}$ & - & $\sum(1-12) \times 3$ \\
\multicolumn{1}{c}{ Cohort III } & - & - & $\mathbf{3}$ & - & - & $\mathbf{6}$ & - & & $\mathbf{9}$ & - & - & $\mathbf{1 2}$ & \\
\hline
\end{tabular}

* printed in bold are the months for which true costs were used; IM = individual mean; LOCF = last observation carried forward; NOCB = next observation carried backward

\section{ANALYSIS}

To examine the frequency of different types of healthcare utilization, we analyzed "true volumes" of various categories of healthcare utilization by means of descriptive statistics and assessed which simulation would be preferable. We first compared "true volumes" with "simulated volumes" for each category of healthcare utilization by means of correlations, and then compared "true annual costs" with "simulated annual costs". Despite the fact that the cost distribution is usually skewed, arithmetic means are generally considered the most appropriate measures to describe cost data ${ }^{23,24}$, because decision makers are interested in the total costs of treating all patients as well as in those for persons consuming a disproportionate volume of healthcare. For this reason, we analyzed the mean difference between "true annual costs" and "simulated annual costs", and used paired T-tests to compare the means. Because previous studies had taught us that collecting data over shorter periods (periodically) increases the variance of the estimate ${ }^{2,13,14}$, we also present the standard deviation. Finally, we assessed the correlations between "true annual costs" and "simulated annual costs" by means of parametric (Pearson) and non-parametric (Spearman) correlations. 


\section{RESULTS}

\section{Participants}

Analyses for the present study were restricted to those participants with complete data on resource utilization in the trial $(n=190)$. The mean age of the participants was $74.5 ; 128$ participants $(67 \%)$ were female; $83(44 \%)$ were living alone; $48(25 \%)$ had completed primary education or less; and $69(36 \%)$ had experienced a major injury (i.e. fracture or joint dislocation) from the fall for which they attended the A\&E department.

\section{Volumes and estimated annual health expenditures}

Table 2 shows the cost price per unit of each category of healthcare utilization in column 2. Table 2 also shows descriptive statistics of true annual volumes (based on continuous measurements during one year) per category of healthcare utilization for the total group $(n=190)$ in columns 3 to 6 , listing mean, standard deviation, minimum, and maximum, respectively. Since low mean volumes correspond with infrequent events, table 2 shows that most infrequent events were expensive. Moreover, all extremely expensive events, costing $€ 1000$ or over, happened on average fewer than 0.1 times per person per year (see also the maximum in Table 2). Most categories of healthcare utilization showed a standard deviation of volumes below 1, although some categories had an extremely high standard deviation. 
Table 2 Categories of healthcare utilization: cost prices per unit and descriptives of annual volumes $(n=190)$

\begin{tabular}{|c|c|c|c|c|c|}
\hline \multirow[t]{2}{*}{ Category of healthcare utilization } & \multirow{2}{*}{$\begin{array}{l}\text { Cost } \\
\text { price }\end{array}$} & \multicolumn{4}{|l|}{ Volumes } \\
\hline & & Mean & (sd) & Min & $\overline{\operatorname{Max}}$ \\
\hline$\overline{\text { General Practitioner * }}$ & $€ 25$ & 7.52 & $(6.56)$ & 0 & $\overline{42}$ \\
\hline Hospital stay (days) ${ }^{\#}$ & $€ 495$ & 0.99 & $(3.23)$ & 0 & 25 \\
\hline Specialty care $*$ & $€ 104$ & 7.05 & $(9.05)$ & 0 & 90 \\
\hline Allied health professional * & $€ 41$ & 12.23 & $(19.59)$ & 0 & 91 \\
\hline Social meal catering service ${ }^{\#}$ & $€ 5$ & 11.04 & $(48.44)$ & 0 & 318 \\
\hline Ambulance transport ${ }^{\#}$ & $€ 460$ & 0.01 & $(0.07)$ & 0 & 1 \\
\hline Informal and private help $\$$ & $€ 9$ & 75.83 & $(172.40)$ & 0 & 1150 \\
\hline Professional help $\$$ & $€ 35$ & 63.09 & (145.59) & 0 & 1451 \\
\hline Walking cane ${ }^{\#}$ & $€ 16$ & 0.17 & $(0.58)$ & 0 & 4 \\
\hline Rolling walker or zimmer frame ${ }^{\#}$ & $€ 106$ & 0.13 & $(0.47)$ & 0 & 3 \\
\hline Wheelchair" & $€ 416$ & 0.03 & $(0.20)$ & 0 & 2 \\
\hline Orthopedic footwear ${ }^{\#}$ & $€ 697$ & 0.21 & $(0.76)$ & 0 & 6 \\
\hline Support stockings, arch support, hip protector ${ }^{\#}$ & $€ 70$ & 0.21 & $(0.59)$ & 0 & 4 \\
\hline Mobility scooter $\#$ & $€ 911$ & 0.01 & $(0.07)$ & 0 & 1 \\
\hline Lift chair ${ }^{\#}$ & $€ 1,414$ & 0.01 & $(0.07)$ & 0 & 1 \\
\hline Hearing device ${ }^{\#}$ & $€ 456$ & 0.02 & $(0.16)$ & 0 & 2 \\
\hline Minor home adaptations (e.g. handrails) ${ }^{\#}$ & $€ 60$ & 0.96 & $(2.08)$ & 0 & 13 \\
\hline Medium-size home adaptations (e.g. raised toilet seat) ${ }^{\#}$ & $€ 350$ & 0.36 & $(1.05)$ & 0 & 7 \\
\hline Major home adaptations (e.g. adapted bathroom) ${ }^{\#}$ & $€ 3,600$ & 0.08 & $(0.37)$ & 0 & 3 \\
\hline
\end{tabular}

${ }^{*}$ consultation; ${ }^{*}$ number; ${ }^{\$}$ hours

Table 3 shows the highest and lowest correlations of "simulated volumes" with "true volumes" for each individual category of healthcare utilization. For most categories, the measurement pattern with the lowest correlation was SIM2, followed by SIM1. SIM3 showed the highest correlation in almost all categories, while never resulting in the lowest correlation.

The imputation method having the lowest correlation with "true volumes" for each category of healthcare utilization was in most cases LOCF (lastobservation-carried-forward) followed by NOCB (next-observation-carriedbackward). The IM (individual mean) imputation method had the highest correlation with "true volumes" in all categories.

The combination of SIM3 (i.e. random cohort method) with individual mean (IM) imputation generally had the highest correlation with "true volumes", and never had the lowest correlation with "true volumes" in any category of healthcare utilization. When SIM3/IM did not show the highest correlation with "true volumes", its correlation was only slightly lower $(<0.08)$ than that of the simulation with the highest correlation for that category of healthcare utilization (see footnote to table 3). 
Table 3 Correlation of worst and best simulation with true volumes of healthcare utilization

\begin{tabular}{|c|c|c|c|c|}
\hline \multirow[t]{2}{*}{ Category of Healthcare utilization } & \multicolumn{2}{|c|}{ LOWEST CORRELATION } & \multicolumn{2}{|c|}{ HIGHEST CORELATION } \\
\hline & Simulation & Pearson* & Simulation & Pearson* \\
\hline General Practitioner consultation & SIM1 LF & .68 & SIM3 IM & .87 \\
\hline Days of hospital stay & SIM1 LF & .60 & SIM1 IM & $.87^{\# 1}$ \\
\hline Specialty care & SIM1 LB & .66 & SIM3 IM & .92 \\
\hline Allied health professional consultation & SIM2 LB & .78 & SIM3 IM & .95 \\
\hline Social services meal catering & SIM2 LF & .92 & SIM3 IM & .99 \\
\hline Ambulance & $x$ & $x$ & $\mathrm{x}$ & $x$ \\
\hline Informal and private help & SIM2 LB & .68 & SIM2 IM & $.87^{\# 2}$ \\
\hline Professional help & SIM1 LF & .76 & SIM2 IM & $.95^{\# 3}$ \\
\hline Walking cane & SIM2 LF & .72 & SIM2 IM & $.83^{\# 4}$ \\
\hline Rolling walker or zimmer frame & SIM2 LF & .57 & SIM2 IM & $.85^{\# 5}$ \\
\hline Wheelchair & SIM2 IM & .50 & SIM2 LB/IM & $.88^{\# 6}$ \\
\hline Orthopedic footwear & SIM2 LF & .43 & SIM3 IM & .72 \\
\hline $\begin{array}{l}\text { Support stockings, arch support, hip } \\
\text { protector }\end{array}$ & SIM2 LF & .49 & SIM3 IM & .77 \\
\hline Mobility scooter & $\mathrm{x}$ & $x$ & $\mathrm{x}$ & $\mathrm{x}$ \\
\hline Lift chair & $\mathrm{x}$ & $x$ & $x$ & $x$ \\
\hline Hearing device & SIM1 IM/LB/LF & .43 & SIM3 IM & .89 \\
\hline Minor home adaptations & SIM2 LB & .43 & SIM2 IM & $.66^{\# 7}$ \\
\hline Medium-size home adaptations & SIM2 LF & .46 & SIM3 IM & .84 \\
\hline major home adaptations & SIM2 LF & .30 & SIM3 IM & .72 \\
\hline
\end{tabular}

* Pearson correlation coefficient; ${ }^{*}$ Corresponding SIM3/IM correlation in this cost category was ${ }^{\# 1}(.86),{ }^{\# 2}(.86),{ }^{\# 3}(.92),{ }^{\# 4}(.75),{ }^{\# 5}(.78),{ }^{\# 6}(.86),{ }^{\# 7}(.65) ; X$ cannot be computed because at least one of the variables is constant

Table 4 shows that there was no significant difference between mean annual costs measured continuously over the entire year ("true annual costs") and mean annual costs estimated from data for limited periods (at least three months), regardless of the periods in which costs were measured or the imputation method used. SIM2/LOCF resulted in an underestimation of the annual costs, while all other simulations resulted in an overestimation. Furthermore, all simulations showed a high correlation with "true annual costs" when analyzed with Pearson (all > .78) as well as with Spearman (all > .87, not tabulated). Individual mean imputation of both SIM2 and SIM3 resulted in the highest correlation with "true costs". SIM3/IM had the smallest difference in mean "true" and mean simulated annual cost, without much increase in standard deviation corresponding to only a slight increase in variance. 
Table 4 Comparison of true annual cost with simulated annual costs

\begin{tabular}{|c|c|c|c|c|}
\hline & $\begin{array}{c}\text { Mean annual (sd) } \\
\text { costs }(€)\end{array}$ & $\begin{array}{l}\text { Mean difference } \\
\text { with true cost }(€)\end{array}$ & $\begin{array}{c}\text { P-values Paired } \\
\text { T-test }\end{array}$ & $\begin{array}{c}\text { Pearson } \\
\text { Correlation * }\end{array}$ \\
\hline True annual costs & 5534 (7403) & 0 & - & 1 \\
\hline \multicolumn{5}{|c|}{ Simulated annual costs } \\
\hline SIM1 IM & 6317 (10553) & 783 & .05 & .88 \\
\hline SIM1 LOCF & 6012 (10370) & 478 & .29 & .80 \\
\hline SIM1 NOCB & 6319 (11951) & 785 & .14 & .81 \\
\hline SIM2 IM & 5899 (8691) & 365 & .15 & .92 \\
\hline SIM2 LOCF & 5150 (7249) & -384 & 18 & .86 \\
\hline SIM2 NOCB & 6057 (12217) & 523 & .37 & .78 \\
\hline SIM3 IM & 5789 (8717) & 255 & .34 & .91 \\
\hline
\end{tabular}

$* p=0.000$ for all comparisons

\section{DISCUSSION}

Our analyses confirmed that a periodic cost diary (measuring in at least three different months during one year) provides a good estimate of annual health expenditures, regardless of the measurement patterns and imputation techniques used. However, when assessing healthcare utilization per category, the measurement patterns and imputation methods used to calculate annual cost data seem to have a considerable influence on the outcomes.

The best measurement pattern seems to be random cohort measurement (SIM3), for both total annual costs and volumes per category of healthcare utilization. It is reasonable to assume that this pattern decreased the risk of missing sudden changes in health expenditures, for example due to the course of a disease, intervention influences, and seasonal influences, ${ }^{2,8,13,14,17}$ as this is the only pattern that collects data throughout the year (with at least one third of the participants measuring costs in each month). That SIM3 seems to be the best measurement pattern is confirmed by the fact that SIM3 showed the best correlation with "true" volumes for the largest number of categories of healthcare utilization.

The best imputation method according to our results is IM (individual mean imputation). This seems plausible, as table 1 shows that in $I M$, the relative weight of each month of measurements (e.g. months 1, 6, and 12 in SIM1) is equally important for the calculation of annual costs, whereas in LOCF some measurement months might have a higher relative weight in the calculation of annual costs (e.g. months 1 and 6 for LOCF in SIM1 and months 6 and 12 for 
NOCB in SIM1). In short, every measured month has the same importance in the calculation of annual costs in IM. Our results also revealed that, although frequently used, LOCF seems to be the least preferred imputation technique for this purpose.

Our analyses of the "true annual volumes" (the total of twelve months of continuous data) confirmed that events causing extremely expensive healthcare utilization ( $>€ 1000$ ) were rare (less than 0.1 times per person per year). These events are therefore likely to be missed in periodic measurements, which may lead to an underestimation of the annual costs. However, our results show that this problem is limited, because we only found a small underestimation in one of the simulations (SIM2/LOCF).

This study had some limitations. Firstly, we limited our analyses to single imputation methods, although multiple imputation techniques are generally preferred. We did this, however, because we intended to use feasible methods for periodic cost assessments. ${ }^{10-12}$ Moreover, since the single imputation methods yielded high correlations with "true annual costs", we believe that multiple imputation methods would not yield much better results. Secondly, we ignored cost distributions, although different cost distributions might have an impact on periodic measurement methods. Further studies are needed to assess the effects of measurement patterns and imputation methods in different cost distributions, such as normal or poisson distributions. A third limitation of the present study was that we restricted our analyses to complete cases in a study with elderly people, so persons who withdrew from the trial were not available for these analyses. Mean baseline characteristics of the complete cases were not different from those of all 333 persons originally included in the RCT. ${ }^{25}$ However, it is possible that persons who withdrew because of health reasons had a different pattern of healthcare expenditures, which may have had some influence on the outcomes of the imputations. Fourth, it needs to be considered that the lack of differences between "true costs" and "simulated costs" may be due to the small sample size $(n=190)$. Finally, we only tested our assumptions by the data of one study, a fall prevention trial. Although we think that it may also be applied to patients with other conditions, this needs further exploration.

Our study revealed that the periods (months) in which costs are measured and the methods used to extrapolate the periodic data to full annual volumes have consequences for the cost calculation per category of healthcare 
utilization. We also conclude that LOCF imputation methods should be avoided.

Remarkably, published reports on trials do not always clearly describe measurement patterns and imputation methods. ${ }^{14,17-19}$ We therefore recommend that the patterns and imputation methods should always be reported in detail.

Our study confirmed that periodic data collection often increases the variance of the annual costs. To overcome this problem, researchers might consider including more participants when using discontinuous cost diaries to collect data. ${ }^{2,14}$ In conclusion, to relieve participants from the burden of continuously filling out diaries over a long follow-up period, we recommend that future economic evaluations use measurement patterns in random cohorts (SIM3) combined with individual mean imputation, as this yields the best estimate of annual costs based on periodic cost diaries, and may prevent missing values or attrition. Additionally, if one extremely expensive event is expected to occur, it might be useful to use a questionnaire to obtain information about the occurrence of this event, because recall bias is not likely to occur for these extremely expensive events.

\section{REFERENCES}

1. Polder JJ, Achterberg PW. Cost of illness in the Netherlands. Highlights. Bilthoven: National Institute for Public Health and the Environment, 2004: 1-19.

2. Goossens ME, Rutten van Molken MP, Vlaeyen JW, van der Linden SM. The cost diary: a method to measure direct and indirect costs in cost-effectiveness research. J Clin Epidemiol 2000;53(7):688-95.

3. Van den Brink M, van den Hout WB, Stiggelbout AM, van de Velde CJ, Kievit J. Cost measurement in economic evaluations of health care: whom to ask? Med Care 2004;42(8):740-6.

4. Tisnado DM, Adams JL, Liu H, et al. What is the concordance between the medical record and patient self-report as data sources for ambulatory care? Med Care 2006;44(2):132-40.

5. Drummond MF, Sculpher MJ, Thorrance GW, O'Brien BJ, Stoddart GL. Methods for the Economic Evaluation of Health Care Programmes. Third ed. New York: Oxford University Press, 2005.

6. Van den Brink M, van den Hout WB, Stiggelbout AM, Putter H, van de Velde CJ, Kievit J. Selfreports of health-care utilization: diary or questionnaire? Int J Technol Assess Health Care 2005; 21(3):298-304.

7. Freer CB. Health diaries: a method of collecting health information. $J R$ Coll Gen Pract 1980; 30(214):279-82.

8. Richardson A. The health diary: an examination of its use as a data collection method. J Adv Nurs 1994;19(4):782-91.

9. Verbrugge LM. Health diaries. Med Care 1980;18(1):73-95. 
10. Manca A, Palmer S. Handling missing data in patient-level cost-effectiveness analysis alongside randomised clinical trials. App/ Health Econ Health Policy 2005;4(2):65-75.

11. Burton A, Billingham LJ, Bryan S. Cost-effectiveness in clinical trials: using multiple imputation to deal with incomplete cost data. Clin Trials 2007;4(2):154-61.

12. Briggs A, Clark T, Wolstenholme J, Clarke P. Missing. .. presumed at random: cost-analysis of incomplete data. Health Econ 2003;12(5):377-92.

13. Lamoureux EL, Chou SL, Larizza MF, Keeffe JE. The reliability of data collection periods of personal costs associated with vision impairment. Ophthalmic Epidemiol 2006;13(2):121-6.

14. Decock RC, Depoorter AM, De Graeve D, Colebunders R. Direct costs of health care for HIV/AIDS patients in Belgium. AIDS Care 2001;13(6):721-31.

15. Verhagen EA, van Tulder M, van der Beek AJ, Bouter LM, van Mechelen W. An economic evaluation of a proprioceptive balance board training programme for the prevention of ankle sprains in volleyball. Br J Sports Med 2005;39(2):111-5.

16. Fiddelers AA, van Montfoort AP, Dirksen CD, et al. Single versus double embryo transfer: costeffectiveness analysis alongside a randomized clinical trial. Hum Reprod 2006;21(8):2090-7.

17. Norman GR, McFarlane AH, Streiner DL, Neale K. Health diaries: strategies for compliance and relation to other measures. Med Care 1982;20(6):623-9.

18. Ostelo RW, Goossens ME, de Vet HC, van den Brandt PA. Economic evaluation of a behavioralgraded activity program compared to physical therapy for patients following lumbar disc surgery. Spine 2004;29(6):615-22.

19. Geraets JJ, Goossens ME, de Bruijn CP, et al. Cost-effectiveness of a graded exercise therapy program for patients with chronic shoulder complaints. Int J Technol Assess Health Care 2006; 22(1):76-83.

20. Hendriks MR, van Haastregt JC, Diederiks JP, Evers SM, Crebolder HF, van Eijk JT. Effectiveness and cost-effectiveness of a multidisciplinary intervention programme to prevent new falls and functional decline among elderly persons at risk: design of a replicated randomised controlled trial [ISRCTN64716113]. BMC Public Health 2005;5(1):6.

21. Oostenbrink JB, Bouwmans CAM, Koopmanschap MA, Rutten FFH. Handleiding voor kosten onderzoek; methoden en standaard kostprijzen voor economische evaluaties in de gezondheidszorg (Geactualiseerde versie) [Dutch manual for costing: methods and standard costs for economic evaluations in healthcare]. Diemen: College voor zorgverzekeringen, 2004.

22. Oostenbrink JB, Koopmanschap MA, Rutten FF. Standardisation of costs: the Dutch Manual for Costing in economic evaluations. Pharmacoeconomics 2002;20(7):443-54.

23. Ramsey S, Willke R, Briggs A, et al. Good research practices for cost-effectiveness analysis alongside clinical trials: the ISPOR RCT-CEA Task Force report. Value Health 2005;8(5):521-33.

24. Thompson SG, Barber JA. How should cost data in pragmatic randomised trials be analysed? BMJ 2000;320(7243):1197-200.

25. Hendriks MR, Bleijlevens $M H$, van Haastregt JC, et al. Effects of a multidisciplinary fall prevention programme in elderly people at risk: a randomized controlled trial [ISRCTN64716113]. Submitted: available upon request 2007. 

GENERAL DISCUSSION | 129

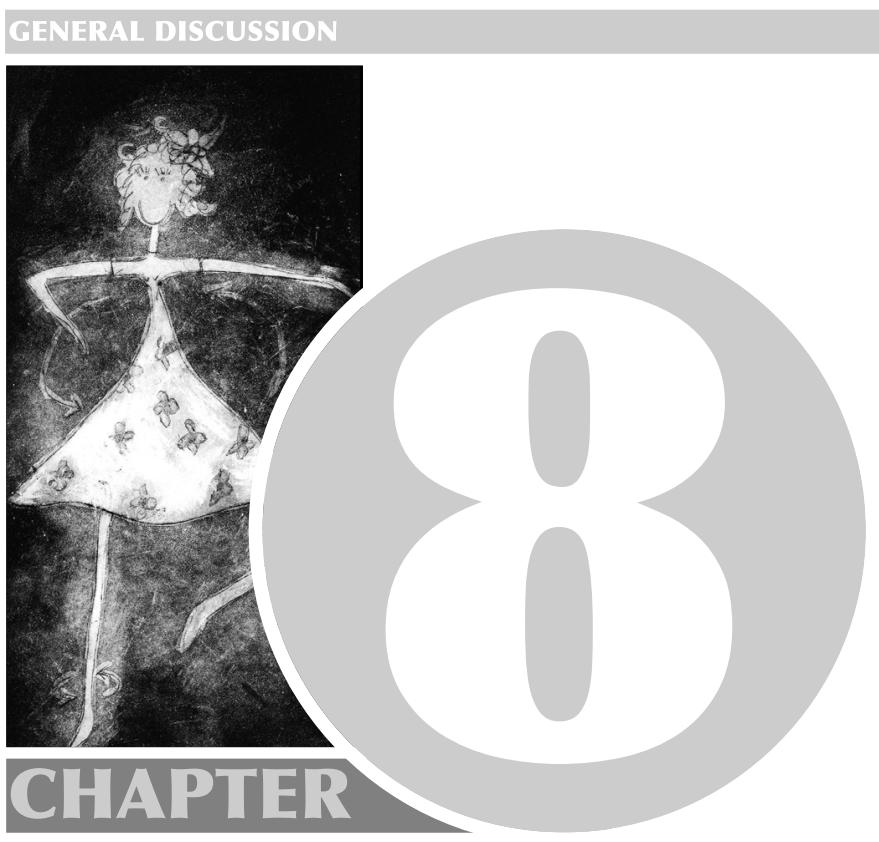





\section{INTRODUCTION}

Since falls are a major health problem among elderly people, fall prevention research has received much attention in recent decades ${ }^{1-3}$ At the start of the research project reported on in this thesis, evidence suggested that effective fall prevention could especially be achieved by programmes involving multifaceted, patient-centred interventions for high-risk populations. ${ }^{1,4}$ One convincing example of such a programme was the medical and occupationaltherapy programme developed by Close and colleagues, which was aimed at elderly persons attending an Accident and Emergency (A\&E) department after an injurious fall. ${ }^{4}$ This programme showed promising effects in terms of both falls and daily functioning. ${ }^{4}$ Since the effectiveness of a programme in one healthcare setting is no guarantee that it will also be effective in another, we decided to evaluate this successful British programme in the Netherlands after adapting it for use in the Dutch setting. ${ }^{5}$

The main objective of this thesis is to explore the effectiveness, feasibility and cost-effectiveness of the medical and occupational-therapy programme to prevent falls and functional decline among elderly people at risk, compared to usual healthcare in the Netherlands. The research project consisted of five parts, whose objectives are summarized below

1. To develop a Dutch version of a successful British multifactorial intervention programme to prevent falls among elderly people.

2. To assess the effectiveness of the adapted version of this fall prevention programme in the Netherlands in terms of falls, functional decline and a number of secondary outcomes.

3. To assess the feasibility of the adapted Dutch version of the fall prevention programme for elderly persons and implementers.

4. To assess the cost-effectiveness of the adapted version of the fall prevention programme in the Netherlands, from a broad societal perspective.

5. To assess the best way to calculate annual costs when health expenditures are measured discontinuously.

This chapter summarizes the main findings of the studies described in the previous chapters of this thesis. This is followed by some theoretical and methodological considerations. Finally, implications and recommendations for practice, policy and future research are given. 


\section{MAIN FINDINGS OF THE THESIS}

In order to adapt the programme developed by Close to Dutch healthcare, we gave General Practitioners (GPs) a central role in implementing the recommendations and referrals resulting from the medical assessment. ${ }^{5}$ Additionally, we concluded from the pilot study that to improve expertise in assessing feet and footwear a rehabilitation physician should cooperate in the medical assessment to improve the available expertise in assessing feet and footwear, and a geriatric nurse should assist the geriatrician to improve efficiency. ${ }^{5}$ The adapted programme was evaluated in a two-group randomized controlled trial (RCT).

The RCT (chapter 4) revealed that the Dutch programme did not result in a decrease in the number of persons who sustained a fall compared to usual care, nor did the time to the first fall differ between the intervention and control groups. In addition, neither daily functioning nor secondary outcome measures proved different between the intervention and control groups. Per protocol analyses and complete case analyses yielded comparable results.

A process evaluation (chapter 5) revealed that the medical and occupationaltherapy programme was acceptable and feasible for both practitioners and participants. Furthermore, the implementers reported that the assessments had been performed largely according to protocol. Although the majority of the participants reported that they had benefited from the programme, the number of referrals and recommendations following the medical assessment was smaller than we expected. Furthermore, the participants' self-reported compliance with the aspect of contacting their GP about the referrals and recommendations resulting from the medical assessment was moderate to low. On the other hand, when participants actually contacted their GP and received the referrals and recommendations from their GP, their self-reported compliance was reasonable to good.

Our economic evaluation (chapter 6) revealed that the programme did not result in higher healthcare costs than usual care. ${ }^{6}$ Overall, mean annual costs were comparable in the intervention and control groups. However, since no difference in effects on falls, daily functioning or quality of life was found between the intervention and control groups, the programme was not costeffective compared to usual care. 
Our economic evaluation was performed from a societal perspective, and cost data were collected continuously from the participants. This proved very labour-intensive and also imposed quite a burden on the participants. Therefore, if we were to plan another trial, we would prefer to measure discontinuously, as many other trials do. ${ }^{7-9}$ However, we found no evidence in the literature regarding the best way to calculate annual costs when measuring health expenditures discontinuously. Our analyses of complete continuous cost-diary data versus simulated discontinuous cost-diary data showed that a periodic cost diary (measuring in at least three months a year) provides a good estimate of annual health expenditures. Moreover, the best estimate of annual costs can be obtained by random cohort measurement, using three random cohorts, ensuring that at least a third of the participants are measuring costs each month, combined with individual mean imputation (for further details see chapter 7).

\section{METHODOLOGICAL AND THEORETICAL CONSIDERATIONS}

\section{Comparison with previous findings and current knowledge}

Fall prevention research has progressed since we started our study. Gillespie and colleagues updated their systematic review in 2003 and maintained their conclusion that multidisciplinary, multifactorial health/environmental risk screening and intervention programmes are likely to be beneficial, especially when individually tailored and delivered by a health professional. ${ }^{1}$ A subsequent systematic review and meta-analysis by Chang and colleagues $(2004)^{2}$ agreed that multifactorial fall risk assessment and management programmes are most effective. In addition, they found that exercise programmes are effective in reducing falls. ${ }^{2}$ In line with this, Kannus and colleagues concluded in 2005 that many reviews, meta-analyses and RCTs corroborate that falls can be prevented by multiple interventions addressing many intrinsic and extrinsic factors simultaneously. ${ }^{3}$ However, based on the reviews by $\mathrm{Chang}^{2}$ and herself $^{1}$, in 2004 Gillespie noted that the reductions in falls that are achieved by these programmes are only moderate, even in the somewhat artificial and perhaps optimal settings of randomized controlled trials. ${ }^{10}$ Very recently (2008), Gates and colleagues performed a systematic review of multifactorial assessment and prevention programmes. ${ }^{11}$ They agreed that fall risk assessment and intervention would appear an attractive solution for fall prevention, but they had found little evidence to support the effectiveness of multifactorial interventions to prevent falls in older people in community and emer- 
gency care settings. Only two of the eighteen trials they reviewed found statistically significant favourable effects on falls and injuries, and one trial even found adverse effects. Subgroup analysis revealed that a greater reduction in falls was found in interventions with higher intensity, but the difference was only of borderline significance, so further studies would be required. ${ }^{11}$ The findings of our trial fit in with this line of thought, as we found that multidisciplinary assessment followed by referral and recommendations (if indicated) was not effective in preventing falls and functional decline. It remains unclear, however, whether direct intervention would have been implementable and effective in our setting.

\section{Fall prevention programme}

Three aspects of the present fall prevention programme need to be considered here: the small numbers of referrals and recommendations following the medical assessment, the consequences of involving GPs, and exercise as a possible intervention component.

The small numbers of referrals and recommendations resulting from the medical assessment may have been caused by several factors. The first is that we may not have selected the high-risk group we expected to select. Our population was relatively young compared to that in Close's study (74.9 versus 78.2 years). ${ }^{4}$ Moreover, only a small number of participants died compared to the British study $(n=6,2 \%$ versus $n=46,12 \%)$, and none of the participants moved into a home for the elderly or nursing home during our trial in contrast to 36 participants in Close's trial $(n=18,8 \%$ of the control group, and $\mathrm{n}=18,10 \%$ of the intervention group). ${ }^{4}$ However, the number of recurrent fallers in our control group was comparable to that in previous studies. ${ }^{4,12,13}$ It is therefore unlikely that this factor explains the lack of effects found in our study. Another explanation for the small number of referrals and recommendations resulting from the medical assessment is that our participants were initially at risk but were already receiving sufficient care, so that potential risk factors were already being detected and addressed. However, the fact that the number of recurrent fallers in our control group was comparable to that in previous studies indicates that usual care in our study is not substantially better in preventing falls than previous studies.

In addition to the small numbers of referrals or recommendations following the medical assessment, the involvement of GPs had some negative consequences for our programme. Firstly, involving the GP extended the period 
that elapsed between the fall and the actual intervention or the time when action was taken. It is therefore possible that persons already sustained a new fall before the risk factors had been addressed. Secondly, only half of the participants asked their GP about the results and recommendations, and therefore only a limited number of recommendations or referrals actually reached the patients. Thirdly, GPs did not follow all suggestions made by the geriatrician. We do not know the reasons for not following the geriatrician's suggestions, but the intended referrals or recommendations may already have been covered by regular healthcare. Furthermore, the GPs' failure to follow all of the geriatrician's suggestions may also be explained by the fact that physicians are generally autonomous care providers who are used to deciding about the most appropriate care or referral for their patients. ${ }^{14}$ The GPs in our study were expected to follow the advice of the geriatrician without having been involved in the assessments, which may have resulted in some resistance on the part of the GPs involved. Finally, the GPs may simply not have had enough time for fall prevention. ${ }^{15,16}$ Overall, although the involvement of the GPs seemed an essential adaptation to implement the programme in regular healthcare in the Netherlands, this appeared to be inefficient, and is likely to have contributed to the lack of effectiveness of our programme.

The final issue to be discussed with regard to our programme is the fact that it did not explicitly address exercise. It has often been suggested that exercise is an important element of multifactorial fall prevention programmes. ${ }^{1,2}$ In our study, none of the participants was recommended to join an exercise programme. It is possible that this aspect was not addressed because it was not incorporated explicitly in the assessment protocol and therefore the implementers simply did not consider referral to exercise. However, it is also possible that our study population was not in need of exercise.

\section{Usual care}

Usual care was the comparator in our study, as in many previous fall prevention studies. However, fall prevention research has progressed in recent years, and several guidelines for preventing falls have been developed and implemented..$^{17-19}$ Therefore, usual care may have improved compared to the usual care in earlier studies. This may have made it more difficult to show effects of our programme, because it may have reduced the contrast between programme and usual care compared to a decade (or more) ago. We have no clear indications that usual care has improved over the years. In general, no standard approach to fall risk assessment is used for fallers presenting to the 
A\&E department and being discharged home. ${ }^{20-22}$ Furthermore, one in three elderly persons still fall each year, and the finding that the fall risk in our study population was similar to that in previous studies, seem to indicate that improved usual care is unlikely to explain the ineffectiveness of our programme. ${ }^{1,23}$ However, it also indicates that it is rather difficult to influence fall rates among elderly persons.

\section{LIMITATIONS OF THE STUDY}

Limitations of our study are related to design of the main study, outcome measures and analyses of the periodic cost diary.

\section{Design}

A limitation of the design of our study may be that some of the GPs involved in the trial had patients from both the intervention and control groups. Consequently, we cannot exclude that the programme also influenced the way they treated the control patients. However, the referrals and recommendations following the assessments were tailored to individual patients. Moreover, GPs were not aware which patients had been allocated to the control group. Hence, we regard a contamination effect as unlikely.

Although the process evaluation provided insights into several factors which may have contributed to the ineffectiveness of our programme, some aspects need to be considered. Firstly, the process evaluation may have been influenced by socially desirable answers. Although we tried to avoid this by collecting data anonymously from the participants and explaining to both participants and practitioners that their answers would not affect their future treatment or work, we cannot exclude the possibility that they suppressed certain negative aspects they perceived. Secondly, we did not gather data from the GPs. The GPs had an important role in implementing the referrals and recommendations following the assessments, and it remains important to ask them for their opinions about the programme. ${ }^{24}$ It would have been useful to explore why some referrals or recommendations were not followed by the GPs.

A limitation of our economic evaluation was that measurements were restricted to one year after baseline, which is relatively short to assess aspects like admission to nursing homes. Falls are a common risk factor for nursing 
home admissions, ${ }^{25}$ which are associated with substantial costs. ${ }^{26}$ However, during the twelve-month follow-up of our trial, no one was admitted to a nursing home. We may have missed nursing home admissions as they happened after the end of our follow-up period. Furthermore, data collection on resource use in our trial was not specifically related to falls, and elderly people's general healthcare utilization is extensive. ${ }^{27}$ This may have made cost differences related to the fall prevention programme difficult to detect. However, it is very difficult to make a distinction between healthcare related to falls and other (unrelated) healthcare, as falls are often a consequence of the interaction of several risk factors. ${ }^{28}$ Moreover, in terms of internal consistency it was appropriate to include unrelated (i.e. generic) healthcare utilization, as we used generic health-related quality of life as an effect measure in our cost-utility analysis. ${ }^{29}$ Finally, presenting generic costs and effects is useful for healthcare decision makers, who aim to maximize health given a fixed budget constraint. ${ }^{29}$

As in previous evaluations of fall prevention programmes, our study focussed primarily on health gains, defined as a reduction of falls, improved daily functioning and improved quality of life. The programme would have been considered successful if it had resulted in health gains (e.g. fewer people sustaining new falls) or if it had accomplished the same health gains at lower costs as a result of decreased healthcare utilization. However, health is not the only potential benefit of innovations in healthcare, as people may also be willing to pay for 'less unpleasant' healthcare, provided the outcomes are equal. ${ }^{30}$ Thus, it is not only the outcomes of new programmes that people value, but also the process of healthcare, something which is referred to as process utility. ${ }^{30}$ Our process evaluation revealed some information about the process of healthcare, for example in that most participants perceived the programme as feasible, pleasant and beneficial. Participants might thus have preferred the programme over usual care. However, we did not study the preferences for process elements, nor the trade-off between the various process elements in our programme and usual care in relation to outcomes like fall reduction.

\section{Main outcome measures}

The validity and reliability of the instruments we used to measure the main outcomes (i.e. falls, daily functioning, health-related quality of life and healthcare utilization) need to be considered. 
Falls were prospectively measured by means of a fall calendar over a period of one year, and participants were phoned monthly to report any falls during the previous month. Although the accuracy of self-report data remains unclear, older people are often the only witnesses of their fall events, so self-reports remain an important source of information about falls. ${ }^{31}$ Furthermore, whereas prospective registration is generally preferred as a method to collect data on falls, the telephone call may have caused retrospective recall, which may have resulted in some recall error in the data. ${ }^{32}$ Nevertheless, since fall calendars are commonly used in fall prevention studies, and therefore increase the comparability of data between fall prevention studies, calendars remain the preferred instrument to collect such data.

We assessed daily functioning by means of the Frenchay Activity Index (FAI), as the study by Close and colleagues ${ }^{4}$ the Barthel Index showed a ceiling effect. The FAI was originally developed for stroke patients, but has proved to be suitable for the general population of elderly people. ${ }^{33,34}$ Moreover, the FAI measures not only activities of daily living (ADL) but also complex activities like housekeeping, recreation, hobbies and social interaction. These so-called instrumental activities of daily living (IADL) may affect the quality of life considerably. ${ }^{33}$

Health-related quality of life in our study was measured by means of the Euroqol. ${ }^{35}$ Although there is some evidence that the Short-Form 36 Health Survey (SF-36) is more sensitive to lower levels of morbidity when used in an elderly population, the Euroqol is sufficiently sensitive for use in elderly people. ${ }^{36}$ Moreover, the Euroqol is short, and results in higher completion rates in the elderly population. ${ }^{36}$

We used a continuous cost diary to measure healthcare utilization, ${ }^{7}$ and participants reported their healthcare utilization during a monthly telephone interview, at the same time reporting the data from their fall calendar. Cost diaries represent a good method to collect data on costs within the formal healthcare sector as well as out-of-pocket expenses for patients (e.g. over-thecounter medication, informal care, travelling expenses). ${ }^{7,37}$ However, number of persons who completed all diaries was low (190,57\%), so completing a cost diary continuously during one year of follow-up revealed a burden to the participants. Therefore, it might be better to use periodic (i.e. discontinuous) cost diaries rather than continuous diaries. 


\section{The periodic cost diary analyses}

Our analysis to compare continuous versus discontinuous measurement used single imputation rather than complex multiple imputation methods. As the methods we used already yielded high correlations, we assume that more complex methods would not have added much information. Secondly, we ignored other possible cost distributions, such as normal or Poisson distributions, so this aspect remains to be explored. Finally, we only tested our assumptions on the data of one study, the fall prevention trial described in this thesis. Although we think that these findings may also be applied to patients with other conditions, this also needs further exploration.

\section{CONCLUSION AND RECOMMENDATIONS}

\section{Conclusion}

Our medical and occupational-therapy programme had no favourable effects on falls and daily functioning among older persons who had sustained an injurious fall. However, the individual and societal burdens of falls and their consequences remain: recent cost-of-illness studies confirm that falls are still associated with substantial costs. ${ }^{26,27,38-42}$ Moreover, these costs are expected to rise further in the coming decades, because demographic changes resulting from the post-war baby boom are causing a growth in the number of elderly people. ${ }^{43}$ For this reason, there remains a great need for effective fall prevention strategies, and fall prevention should be high on the agenda. Our detailed evaluation of a medical and occupational-therapy revealed several aspects that could be optimized to improve future fall prevention programmes. We therefore recommend not to implement the programme in its current form, but to improve several aspects and assess if these adaptations result in greater effectiveness and efficiency. Furthermore, it is important to disseminate the findings of this trial, so as to inform care providers and policy makers.

\section{Recommendations for clinical practice}

We recommend three major adjustments to improve the efficiency and effectiveness of our fall prevention programme. Firstly, the programme should preferably focus on those participants who are at increased risk for recurrent falls. We therefore recommend using a short validated fall risk 
screening tool to distinguish the low- to moderate-risk groups from the highrisk group among community-living fallers presenting at the A\&E department. Several risk profiles, differing in complexity, duration of assessment, predictive value and sensitivity, have been developed. ${ }^{44-48}$ Unfortunately, we found no data on the validation of these tools. We recommend choosing a short and easy-to-use risk profile like the 'desk model' developed by Stalenhoef and colleagues. ${ }^{45}$ Subsequently, the high-risk group thus identified should receive a detailed assessment, immediately followed by intervention tailored to their individual situation. In addition, the low-risk group could receive general information about fall prevention. Secondly, since exercise is often recognized as an important component of multifactorial fall prevention interventions, we recommend adding this to future fall prevention programmes. ${ }^{1-3}$ Finally, we recommend drastically reducing the time that elapses between the moment when a patient attends the A\&E department and the implementation of the fall prevention measures.

In order to improve fall prevention in practice, such programmes need to be feasible, and possible barriers to their implementation should be addressed. ${ }^{14-}$ 16,49 Possible barriers to fall prevention include logistical factors (e.g. the problem of direct referral of non-acute patients, and lack of time), ${ }^{14,15}$ healthcare provider factors (e.g. awareness, competence and motivation) ${ }^{16,50}$ and patient factors (e.g. compliance). ${ }^{14,16}$ In order to decrease the time that elapses between the fall and the moment when potential risk factors are addressed, the geriatrician who performs the medical assessment should be permitted to refer patients directly to relevant services, instead of having the GP implement the referrals. We therefore need to search for opportunities to overcome the barrier of direct referral of non-acute patients. If GPs remain required for referral to secondary care, it is important to communicate with them about their involvement in order to signal and act upon any perceived barriers. Furthermore, participants should be stimulated to comply with the recommendations. We therefore recommend adding a follow-up visit by the assessors, to stimulate compliance by both participants and practitioners.

\section{Recommendations for future research on fall prevention}

Because the proposed procedure (direct referral by geriatricians) deviates from usual procedures in the Netherlands, there is a need to thoroughly explore whether the recommended adaptations to the programme would be effective and cost-effective and feasible in Dutch healthcare. In addition, we have several recommendations to improve future research on fall prevention. 
- It revealed important to assess a programme in the setting in which it will be applied and implemented, and to consider these setting and implementation factors within the evaluation of a programme. ${ }^{51}$ In this aspect, process evaluation revealed important to examine those aspects that may explain the effectiveness or ineffectiveness of a programme. ${ }^{24}$ As these aspects are interrelated with each other, evaluations of new complex programmes (like those in fall prevention) should preferably study effects, feasibility and cost-effectiveness simultaneously, together with implementation factors, in one design. ${ }^{51,52}$

- In addition to health benefits, the utility associated with the treatment process in fall prevention programmes should also be taken into account in resource allocation decisions. ${ }^{53}$ In order to gain information about participants' valuation of these treatment process factors, we therefore recommend studying process utility. ${ }^{37}$ Process utility can for example be estimated by means of a discrete choice experiment which studies the relative importance of different aspects of care and the trade-offs between these aspects, to provide a overall utility that respondents derive from healthcare services. ${ }^{54,55}$

- Possible long-term effects should be examined by extending the followup period in future fall prevention studies to at least two years. Another option would be to build a mathematical model to simulate the costeffectiveness of programmes over a period that exceeds the time horizon of a trial, for example by means of decision trees or micro-simulation models. $^{37}$

- It proved difficult to distinguish fall-related from unrelated healthcare utilization. It would be possible to ask patients which healthcare utilization they perceived to be related to falls or not. Additionally, the same information could be gathered from healthcare providers or insurance companies. Subsequently, sensitivity analysis can be used to compare unrelated healthcare utilization versus fall-related healthcare utilization with effects according to different perspectives. ${ }^{29,56}$

- To relieve participants of the burden of continuous cost diaries, we recommend collecting cost data discontinuously (during at least three months a year), by means of random cohorts, ensuring that at least a third of the participants are measuring costs each month. Subsequently, annual costs can be obtained by individual mean imputation. 


\section{CONCLUDING REMARKS}

Our multidisciplinary fall prevention programme proved feasible but not effective or cost-effective compared to usual care in the Netherlands. Nevertheless, falls still have an important impact on society in terms of costs and effects, and the individual burden is large. We therefore recommend not to implement the programme in its current form, but to improve several aspects and assess if these adaptations result in greater effectiveness and efficiency.

\section{REFERENCES}

1. Gillespie LD, Gillespie WJ, Robertson MC, Lamb SE, Cumming RG, Rowe BH. Interventions for preventing falls in elderly people. Cochrane Database Syst Rev 2003(4):CD000340.

2. Chang JT, Morton SC, Rubenstein LZ, et al. Interventions for the prevention of falls in older adults: systematic review and meta-analysis of randomised clinical trials. BMJ 2004; 328(7441):680.

3. Kannus P, Sievanen H, Palvanen M, Jarvinen T, Parkkari J. Prevention of falls and consequent injuries in elderly people. Lancet 2005;366(9500):1885-93.

4. Close J, Ellis M, Hooper R, Glucksman E, Jackson S, Swift C. Prevention of falls in the elderly trial (PROFET): a randomised controlled trial. Lancet 1999;353(9147):93-97.

5. Hendriks MR, Bleijlevens $M H$, van Haastregt JC, et al. A multidisciplinary fall prevention program for elderly persons: a feasibility study. Geriatric Nursing: accepted for publication; available upon request 2008:000-000.

6. Hendriks MR, Evers SM, Bleijlevens MH, van Haastregt JC, Crebolder HF, van Eijk JT. Costeffectiveness of a multidisciplinary fall prevention program in community dwelling elderly people: a randomized controlled trial [ISRCTN64716113]. International Journal of Technology Assessment in Health Care 2008; (in press).

7. Goossens ME, Rutten van Molken MP, Vlaeyen JW, van der Linden SM. The cost diary: a method to measure direct and indirect costs in cost-effectiveness research. J Clin Epidemiol 2000;53(7):688-95.

8. Decock RC, Depoorter AM, De Graeve D, Colebunders R. Direct costs of health care for HIV/AIDS patients in Belgium. AIDS Care 2001;13(6):721-31.

9. Ostelo RW, Goossens ME, de Vet HC, van den Brandt PA. Economic evaluation of a behavioralgraded activity program compared to physical therapy for patients following lumbar disc surgery. Spine 2004;29(6):615-22.

10. Gillespie L. Preventing falls in elderly people. BMJ 2004;328(7441):653-4.

11. Gates S, Lamb SE, Fisher JD, Cooke MW, Carter YH. Multifactorial assessment and targeted intervention for preventing falls and injuries among older people in community and emergency care settings: systematic review and meta-analysis. BMJ 2008;336(7636):130-3.

12. Tinetti ME, Baker DI, McAvay G, et al. A multifactorial intervention to reduce the risk of falling among elderly people living in the community. N Engl J Med 1994;331(13):821-7.

13. O'Loughlin J, Robitaille Y, Bolvin J-F, Suissa S. Incidence of and risk factors for falls and injurious falls among the community-dwelling elderly. Am J Epidemiol 1993;137:342-54.

14. Chou WC, Tinetti ME, King MB, Irwin K, Fortinsky RH. Perceptions of physicians on the barriers and facilitators to integrating fall risk evaluation and management into practice. J Gen Intern Med 2006;21(2):117-22. 
15. Yarnall KS, Pollak KI, Ostbye T, Krause KM, Michener JL. Primary care: is there enough time for prevention? Am J Public Health 2003;93(4):635-41.

16. Fortinsky $\mathrm{RH}$, lannuzzi-Sucich M, Baker DI, et al. Fall-risk assessment and management in clinical practice: views from healthcare providers. J Am Geriatr Soc 2004;52(9):1522-6.

17. CBO KvdG. Richtlijn preventie van valincidenten bij ouderen. 2004.

18. American Geriatrics Society BGS, and American Academy of Orthopaedic Surgeons Panel on Falls Prevention. Guideline for the prevention of falls in older persons. J Am Geriatr Soc 2001; 49(5):664-72.

19. Feder G, Cryer C, Donovan S, Carter Y. Guidelines for the prevention of falls in people over 65. The Guidelines' Development Group. BMJ 2000;321(7267):1007-11.

20. Salter AE, Khan KM, Donaldson MG, et al. Community-dwelling seniors who present to the emergency department with a fall do not receive Guideline care and their fall risk profile worsens significantly: a 6-month prospective study. Osteoporos Int 2006;17(5):672-83.

21. Russell MA, Hill KD, Blackberry I, Day LL, Dharmage SC. Falls risk and functional decline in older fallers discharged directly from emergency departments. J Gerontol A Biol Sci Med Sci 2006;61(10):1090-5.

22. Donaldson MG, Khan KM, Davis JC, et al. Emergency department fall-related presentations do not trigger fall risk assessment: a gap in care of high-risk outpatient fallers. Arch Gerontol Geriatr 2005;41(3):311-7.

23. Todd C, Skelton D. What are the main risk factors for falls among older people and what are the most effective interventions to prevent falls? Copenhagen: WHO Regional Office for Europe (Health Evidence Network), 2004: 1-28.

24. Oakley A, Strange V, Bonell C, Allen E, Stephenson J. Process evaluation in randomised controlled trials of complex interventions. BMJ 2006;332(7538):413-6.

25. Tinetti ME, Williams CS. Falls, injuries due to falls, and the risk of admission to a nursing home. N Engl J Med 1997;337(18):1279-84.

26. Meerding WJ, Mulder S, van Beeck EF. Incidence and costs of injuries in The Netherlands. Eur J Public Health 2006;16(3):272-8.

27. Polder JJ, Takken J, W.J. M, Kommer GJ, Stokx LJ. Kosten van Ziekten in Nederland. De zorgeuro ontrafeld. Bilthoven: Rijksinstituut voor Volksgezondheid en Milieu, 2002: 1-304.

28. Rubenstein LZ. Falls in older people: epidemiology, risk factors and strategies for prevention. Age Ageing 2006;35 Suppl 2:ii37-ii41.

29. Van Baal PH, Feenstra TL, Hoogenveen RT, de Wit GA, Brouwer WB. Unrelated medical care in life years gained and the cost utility of primary prevention: in search of a 'perfect' cost-utility ratio. Health Econ 2007;16(4):421-33.

30. Donaldson C, Shackley P. Does "process utility" exist? A case study of willingness to pay for laparoscopic cholecystectomy. Soc Sci Med 1997;44(5):699-707.

31. Dickens J, Jones M, Johansen A. Falls definition--reliability of patients' own reports. Age Ageing 2006;35(4):450-1.

32. Hauer K, Lamb SE, Jorstad EC, Todd C, Becker C. Systematic review of definitions and methods of measuring falls in randomised controlled fall prevention trials. Age Ageing 2006;35(1):5-10.

33. Schuling J, de Haan R, Limburg M, Groenier KH. The Frenchay Activities Index. Assessment of functional status in stroke patients. Stroke 1993;24(8):1173-7.

34. Van Haastregt JC, Diederiks JP, van Rossum E, de Witte LP, Voorhoeve PM, Crebolder HF. Effects of a programme of multifactorial home visits on falls and mobility impairments in elderly people at risk: randomised controlled trial. BMJ 2000;321(7267):994-8.

35. Brooks R. Euroqol:the current state of play. Health policy 1996;37(1):53-72.

36. Brazier JE, Walters SJ, Nicholl JP, Kohler B. Using the SF-36 and Euroqol on an elderly population. Qual Life Res 1996;5(2):195-204. 
37. Drummond MF, Sculpher MJ, Thorrance GW, O'Brien BJ, Stoddart GL. Methods for the Economic Evaluation of Health Care Programmes. Third ed. New York: Oxford University Press, 2005.

38. Scuffham P, Chaplin S, Legood R. Incidence and costs of unintentional falls in older people in the United Kingdom. J Epidemiol Community Health 2003;57(9):740-4.

39. Carroll NV, Slattum PW, Cox FM. The cost of falls among the community-dwelling elderly. J Manag Care Pharm 2005;11(4):307-16.

40. Newton JL, Kyle P, Liversidge P, Robinson G, Wilton K, Reeve P. The costs of falls in the community to the North East Ambulance Service. Emerg Med J 2006;23(6):479-81.

41. Stevens JA, Corso PS, Finkelstein EA, Miller TR. The costs of fatal and non-fatal falls among older adults. Inj Prev 2006;12(5):290-5.

42. Roudsari BS, Ebel BE, Corso PS, Molinari NA, Koepsell TD. The acute medical care costs of fallrelated injuries among the U.S. older adults. Injury 2005;36(11):1316-22.

43. Polder JJ, Achterberg PW. Cost of illness in the Netherlands. Highlights. Bilthoven: National Institute for Public Health and the Environment, 2004: 1-19.

44. Stel VS, Pluijm SM, Deeg DJ, Smit JH, Bouter LM, Lips P. A classification tree for predicting recurrent falling in community-dwelling older persons. J Am Geriatr Soc 2003;51(10):1356-64.

45. Stalenhoef PA, Diederiks JP, Knottnerus JA, Kester AD, Crebolder HF. A risk model for the prediction of recurrent falls in community-dwelling elderly: a prospective cohort study. J Clin Epidemiol 2002;55(11):1088-94.

46. Pluijm SM, Smit JH, Tromp EA, et al. A risk profile for identifying community-dwelling elderly with a high risk of recurrent falling: results of a 3-year prospective study. Osteoporos Int 2006; 17(3):417-25.

47. Vassallo M, Stockdale R, Sharma JC, Briggs R, Allen S. A comparative study of the use of four fall risk assessment tools on acute medical wards. J Am Geriatr Soc 2005;53(6):1034-8.

48. Tromp AM, Pluijm SM, Smit JH, Deeg DJ, Bouter LM, Lips P. Fall-risk screening test: a prospective study on predictors for falls in community-dwelling elderly. J Clin Epidemiol 2001;54(8):83744.

49. Tinetti ME, Gordon C, Sogolow E, Lapin P, Bradley EH. Fall-risk evaluation and management: challenges in adopting geriatric care practices. Gerontologist 2006;46(6):717-25.

50. Lin MK, Marsteller JA, Shortell SM, et al. Motivation to change chronic illness care: results from a national evaluation of quality improvement collaboratives. Health Care Manage Rev 2005; 30(2):139-56.

51. Hummel MJ, van Rossum W, Verkerke GJ, Rakhorst G. Assessing medical technologies in development. A new paradigm of medical technology assessment. Int J Technol Assess Health Care 2000;16(4):1214-9.

52. Hoomans T, Severens J, Evers S, Ament A. Value for money of clinical practice change: should decisions about guidelines and implementation strategies be made sequentially or simultaneously? Presented to the Health Economists' Study Group meeting. 2008, University of East Anglia.

53. Drummond MF, McGuire A. Economic Evaluation in Health Care: Merging theory with practice. New York: Oxford University Press, 2004.

54. Ryan M, Farrar S. Using conjoint analysis to elicit preferences for health care. BMJ 2000; 320(7248):1530-3.

55. Ryan M. Discrete choice experiments in health care. BMJ 2004;328(7436):360-1.

56. Ramsey S, Willke R, Briggs A, et al. Good research practices for cost-effectiveness analysis alongside clinical trials: the ISPOR RCT-CEA Task Force report. Value Health 2005;8(5):521-33. 


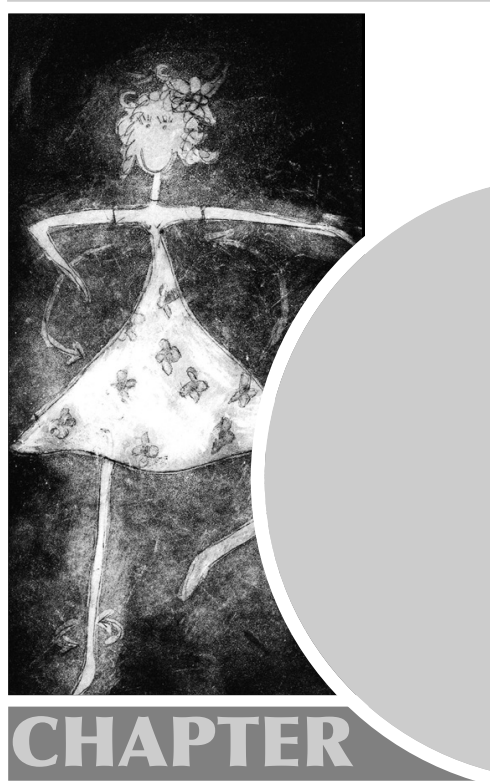

Summary

Samenvatting

Coauthors and affiliations

Curriculum Vitae

Dankwoord 


\section{SUMMARY}

Falls are a common problem among elderly people. About a third of community-living people aged 65 years and over fall at least once a year ${ }^{1,4,31,42-44}$, and people who have sustained a fall are at greater risk of falling again. Falls in elderly people may have several adverse consequences. Around 40 to $70 \%$ result in some kind of physical injury, which may cause decline in mobility or daily functioning. Injurious falls are associated with increased morbidity, which subsequently may cause increased healthcare utilization, resulting in excessive healthcare costs. In addition, falls can also have psychosocial consequences, such as fear of falling, avoidance of activity and social isolation. The need for effective fall prevention strategies is thus evident. As falls are often caused by interactions between several risk factors, multifactorial fall prevention programmes are likely to be beneficial. One convincing example of such a programme is a medical and occupational-therapy programme developed by Close and colleagues, which was aimed at elderly persons attending an Accident and Emergency (A\&E) department after an injurious fall. The programme comprised structured medical and occupational-therapy assessments to identify and address potential risk factors for new falls. This programme showed promising results in the British setting, in terms of preventing falls and functional decline. However, the effectiveness of a programme in one setting is no guarantee that it will also be effective in another setting. We therefore implemented a study to evaluate this programme in the Netherlands.

Chapter 1 presents an overview of the incidence and consequences of falls among elderly people, the aetiology of falls, the prevention of falls and the importance of economic evaluations in fall prevention. The main objective of this thesis is to explore the effectiveness, feasibility and cost-effectiveness of the medical and occupational-therapy programme to prevent falls and functional decline among elderly people at risk, compared to usual Dutch healthcare. This thesis reports on five studies, whose objectives are summarized below.

1. To develop a Dutch version of a successful British multifactorial intervention programme to prevent falls among elderly people.

2. To assess the effectiveness of the adapted version of this fall prevention programme in the Netherlands in terms of falls, functional decline and a number of secondary outcomes. 
3. To assess the feasibility of the adapted Dutch version of the fall prevention programme for elderly persons and implementers.

4. To assess the cost-effectiveness of the adapted version of the fall prevention programme in the Netherlands, from a broad societal perspective.

5. To assess the best way to calculate annual costs when health expenditures are measured discontinuously.

Chapter 2 describes the adaptation of the medical and occupational-therapy programme to make it fit in with the Dutch healthcare setting, as well as a pilot study to assess the feasibility of the adapted programme in the Dutch setting, in the opinion of the implementers of the intervention and the participants. A number of adaptations appeared essential to make the programme fit the Dutch healthcare setting. Since (non-acute) referrals to medical specialists (secondary care) are implemented through a GP (primary care) in the Netherlands, the participants' GPs were given a central role in implementing the referrals and recommendations resulting from the medical assessment. Additionally, we concluded from the pilot study that a rehabilitation physician should cooperate in the medical assessment to improve the available expertise in assessing feet and footwear, and a geriatric nurse should assist the geriatrician to improve efficiency.

Chapter 3 elaborates on the design of the two-group RCT with twelve months of follow-up. The objective of this trial was to assess the effectiveness and cost-effectiveness of the adapted medical and occupational therapy programme in the Netherlands. In addition to the effect evaluation and economic evaluation, a process evaluation was undertaken to assess the feasibility and applicability of the intervention programme for those receiving and implementing the intervention. The target population consisted of communityliving elderly people aged 65 years or over, who had visited an accident and emergency department (A\&E department) or an out-of-hours general practice service (GP cooperative) because of a fall. Participants in the intervention group received the medical and occupational-therapy programme, while those allocated to the control group received usual care. The main outcome measures of the effect evaluation were number of falls and daily functioning. Secondary measures were recuperation from the fall, health complaints, perceived health, activities of daily living (ADL) and instrumental ADL ability, mental health, fear of falling, activity avoidance, social participation and 
quality of life. Cost-effectiveness and cost-utility analyses were performed from a societal perspective, which means that all relevant costs and outcomes were taken into account, regardless of who pays the costs and who benefits from the effects. Falls and healthcare utilization were measured continuously by means of a fall calendar and a cost diary over a period of twelve months. Daily functioning, quality of life and other secondary outcome measures were assessed by means of self-administered questionnaires at baseline and after four and twelve months.

Chapter 4 describes the effect evaluation of the medical and occupationaltherapy programme. Randomization resulted in 166 persons being allocated to the intervention group and 167 persons to the control group. Results showed no statistically significant favourable effects on falls, daily functioning or secondary outcome measures after twelve months of follow-up. Because the medical and occupational-therapy programme was not effective in preventing falls and functional decline in this Dutch healthcare setting, we do not recommend implementing the programme in its present form in the Netherlands.

Chapter 5 reports on the process evaluation that was performed alongside the trial to assess the feasibility of the medical and occupational-therapy programme. Data were collected from participants allocated to the intervention group ( $\mathrm{n}=166)$ and from all practitioners who performed the assessments $(\mathrm{n}=8)$. The programme was found to have been largely carried out according to protocol, and both participants and practitioners judged the programme to be feasible. The number of referrals and recommendations resulting from the medical assessment was small. Participants' self-reported compliance in terms of contacting their GP to be informed of the recommendations and/or referrals was low to moderate, although self-reported compliance with the referrals and/or recommendations they actually received was reasonable to good. A large majority of participants reported they had benefited from the programme. The main factors that seem to be responsible for the lack of effectiveness were the relatively low numbers of referrals and recommendations resulting from the medical assessments and the participants' low compliance as regards contacting their GP to hear about referrals and/or recommendations.

Chapter 6 reports on the economic evaluation that aimed to assess the costeffectiveness of the medical and occupational-therapy programme compared to usual healthcare in the Netherlands. Cost-effectiveness and cost-utility 
analyses were performed from a societal perspective. Bootstrap analyses were used to estimate the uncertainty of the findings, and sensitivity analyses were done to assess the generalizability of the assumptions made. Healthcare and patient and family costs were comparable between the two groups. Since we found no effects on falls, daily functioning or quality of life measures, however, the medical and occupational-therapy programme to prevent falls was not cost-effective compared to usual care in the Netherlands.

Chapter 7 reports on a study that aimed to compare costs derived from discontinuous measurement of healthcare utilization with those derived from continuous measurement. Since continuous measurement of healthcare utilization is often a burden to participants, resulting in missing values or withdrawal, it is important to assess the best way to calculate annual costs when measuring health expenditures discontinuously. In this study we used several simulations of discontinuous measurement patterns, combined with three commonly used imputation techniques: Individual Mean (IM), Last Observation Carried Forward (LOCF) and Next Observation Carried Backward (NOCB). Analyses confirmed that discontinuous measurements using cost diaries yielded good estimates of annual health expenditures, but measurement patterns and imputation methods did influence the outcomes, as the correlations differed between methods. This study revealed that the best estimate of annual costs can be obtained by random cohort measurement, using three random cohorts and ensuring that at least a third of the participants are measuring costs each month, combined with IM imputation.

Chapter 8 discusses the main findings of the studies described in the previous chapters of this thesis. Theoretical and methodological considerations are presented and implications and recommendations are made for practice, policy and future research. The main conclusion of the study presented in this thesis is that the multidisciplinary fall prevention programme proved feasible but not effective or cost-effective compared to usual care in the Netherlands. Nevertheless, falls still have an important impact on society in terms of costs and effects, and the individual burden is large. We therefore recommend not to implement the programme in its current form, but to improve several aspects and assess whether these adaptations result in greater effectiveness and efficiency. We recommend the following adaptations: improved selection of persons with increased fall risk, taking preventive measures more quickly, and adding exercise programmes to fall prevention programmes. Moreover we present several recommendations for future research. 


\section{SAMENVATTING}

Vallen is een veel voorkomend probleem bij ouderen. Ongeveer een derde van de zelfstandig wonende ouderen van 65 jaar en ouder valt één of meer keer per jaar. Ouderen die al eens zijn gevallen, hebben een verhoogd risico om opnieuw te vallen. Vallen bij ouderen heeft diverse negatieve gevolgen. Zo heeft ongeveer 40-70 procent van de valincidenten fysiek letsel tot gevolg, wat vervolgens kan leiden tot mobiliteitsvermindering of slechter dagelijks functioneren. Letsel als gevolg van een val verhoogt vaak ook de kans op morbiditeit, wat kan leiden tot een toename van het gezondheidszorggebruik en daaraan gerelateerde kosten. Verder heeft vallen vaak ook psychosociale gevolgen, zoals angst om te vallen, vermijden van activiteiten en sociale isolatie. Kortom, het belang van effectieve valpreventie is evident.

Vallen wordt meestal veroorzaakt door een combinatie van verschillende risicofactoren. Multifactoriële valpreventie lijkt daarom aangewezen. Een voorbeeld van een dergelijk programma is het programma dat is ontwikkeld door Close en haar collega's. Dat programma was gericht op ouderen die zich met verwondingen ten gevolge van een val hadden gemeld bij de spoedeisende hulp (SEH). Het programma omvatte een gestructureerd medisch en ergotherapeutisch onderzoek om mogelijke risicofactoren voor opnieuw vallen op te sporen en aan te pakken. In de Britse setting liet dit programma veelbelovende resultaten zien voor wat betreft het voorkómen van vallen en het verbeteren van het dagelijks functioneren. Effectiviteit van een programma in een bepaalde setting is echter geen garantie voor effectiviteit in een andere setting. Daarom hebben we het Britse programma aangepast aan de Nederlandse situatie, toegepast en geëvalueerd.

Hoofdstuk 1 geeft een overzicht van de incidentie en de gevolgen van valincidenten bij ouderen, evenals de oorzaken van het vallen, preventie en het belang van economische evaluaties bij valpreventie-onderzoek. Daaruit volgt het doel van het onderzoek waarover dit proefschrift gaat: onderzoek naar de effectiviteit, de praktische uitvoerbaarheid en de kosteneffectiviteit van een medisch-ergotherapeutisch programma ter preventie van vallen en functionele achteruitgang bij ouderen met een verhoogd risico, vergeleken met reguliere zorg in Nederland. Het proefschrift bespreekt vijf studies, met de volgende doelstellingen. 
1. De ontwikkeling van een Nederlandse versie van een succesvol Brits multifactorieel interventieprogramma ter preventie van vallen bij ouderen.

2. Evaluatie van de effectiviteit van het aangepaste valpreventieprogramma voor wat betreft vallen, functionele achteruitgang en een aantal secundaire uitkomstmaten.

3. Evaluatie van de praktische uitvoerbaarheid van het aangepaste valpreventieprogramma voor zowel de ouderen zelf als degenen die het programma moeten uitvoeren.

4. Evaluatie van de kosteneffectiviteit van het aangepaste valpreventieprogramma in Nederland vanuit een breed maatschappelijk perspectief.

5. Onderzoek naar de beste manier om jaarlijkse kosten te berekenen als gezondheidszorggebruik discontinu gemeten wordt.

Hoofdstuk 2 beschrijft de aanpassing van het medisch-ergotherapeutische programma aan de Nederlandse gezondheidszorg en een pilotstudie om te onderzoeken of het programma uitvoerbaar is volgens zowel de uitvoerders als de deelnemers. Een aantal aanpassingen bleek noodzakelijk om het programma te kunnen uitvoeren in de Nederlandse gezondheidszorg. In Nederland worden (niet-acute) verwijzingen naar medische specialisten (tweede lijn) via de huisarts (eerste lijn) geïmplementeerd. Daarom hebben de huisartsen van de deelnemers een centrale rol gekregen bij de uitvoering van verwijzingen en aanbevelingen die resulteerden uit het medische onderzoek. Daarnaast concludeerden we op basis van de pilotstudie dat een revalidatie-arts bij het programma betrokken zou moeten worden, vanwege diens expertise op het gebied van voeten en schoenen, en ook een geriatrisch verpleegkundige, om de efficiëntie van de uitvoering van de metingen te verbeteren.

Hoofdstuk 3 beschrijft de opzet van het twaalf maanden durende gerandomiseerde experimentele onderzoek (RCT) met twee groepen. Het doel van het onderzoek was om de (kosten-)effectiviteit van het aangepaste medischergotherapeutische programma in Nederland te onderzoeken. Naast de effectevaluatie en de economische evaluatie was een procesevaluatie uitgevoerd om de praktische uitvoerbaarheid en toepasbaarheid van het programma te onderzoeken voor zowel degenen op wie de interventie wordt toegepast als degenen die de interventie uitvoeren. De doelpopulatie bestond uit zelfstandig wonende ouderen van 65 jaar of ouder, die zich met de gevolgen van een val hadden gemeld bij de spoedeisende hulp (SEH) of huisartsenpost (HAP). Deelnemers die (willekeurig) waren toegewezen aan de 
interventiegroep namen deel aan het medisch-ergotherapeutische programma. Mensen die waren toegewezen aan de controlegroep ontvingen reguliere zorg. De primaire uitkomstmaten van de effectevaluatie waren het aantal valincidenten en dagelijks functioneren. Secundaire uitkomstmaten waren het herstel na de val, gezondheidsklachten, door de persoon zelf ervaren gezondheid, dagelijkse activiteiten en dagelijks functioneren, mentale gezondheid, angst om te vallen, vermijding van activiteiten, sociale participatie en kwaliteit van leven. Het onderzoek naar de kosteneffectiviteit en kostenutiliteit werd uitgevoerd vanuit een maatschappelijk perspectief, wat inhoudt dat alle relevante kosten en uitkomsten in beschouwing worden genomen onafhankelijk van wie de kosten betaalt of wie voordeel heeft van de effecten. Het aantal valincidenten en het gezondheidszorggebruik werden gedurende twaalf maanden continu gemeten door middel van een valkalender en een kostendagboek. Het dagelijks functioneren, de kwaliteit van leven en andere uitkomstmaten werden gemeten door middel van door de deelnemers zelf ingevulde schriftelijke vragenlijsten vlak voor aanvang van de studie en na vier en twaalf maanden.

Hoofdstuk 4 beschrijft het onderzoek naar het effect van het medischergotherapeutische programma. Door randomisatie werden 166 personen toegewezen aan de interventiegroep en 167 aan de controlegroep. De resultaten na twaalf maanden lieten geen significant gunstige effecten zien ten aanzien van vallen, dagelijks functioneren of secundaire uitkomstmaten. Omdat het door ons uitgevoerde programma niet effectief bleek voor wat betreft het voorkómen van vallen en de achteruitgang in functioneren, is onze aanbeveling om het niet in de huidige vorm in Nederland te implementeren.

Hoofdstuk 5 beschrijft de procesevaluatie die naast de effectevaluatie werd uitgevoerd om de praktische uitvoerbaarheid van het medisch-ergotherapeutische programma te onderzoeken. We verzamelden gegevens van deelnemers die waren toegewezen aan de interventiegroep $(n=166)$ en alle uitvoerders van het medisch-ergotherapeutische programma $(n=8)$. Het programma bleek grotendeels uitgevoerd volgens protocol. Zowel deelnemers als uitvoerders beoordeelden het programma als praktisch uitvoerbaar. Het aantal verwijzingen en aanbevelingen dat voortvloeide uit het medische onderzoek was gering. Het door de deelnemers zelf gerapporteerde opvolggedrag voor wat betreft contact opnemen met de huisarts was gering tot matig. Als de aanbevelingen of verwijzingen echter daadwerkelijk bij de deelnemers terecht kwamen, werden deze volgens de deelnemers redelijk tot 
goed opgevolgd. Een grote meerderheid van de deelnemers gaf aan baat te hebben gehad bij het programma. Factoren die verantwoordelijk lijken voor het uitblijven van een effect zijn het relatief lage aantal aanbevelingen en verwijzingen op basis van het medisch onderzoek en de beperkte mate waarin de deelnemers contact opnamen met de huisarts over mogelijke aanbevelingen en verwijzingen.

Hoofdstuk 6 doet verslag van de economische evaluatie met als doel de kosteneffectiviteit van het medisch-ergotherapeutische programma te vergelijken met die van de reguliere zorg in Nederland. Kosteneffectiviteit en -utiliteit zijn onderzocht vanuit een maatschappelijk perspectief. Met behulp van nonparametrische "bootstrapping"-analyses werd de mate van onzekerheid in de bevindingen geschat, en met sensitiviteitsanalyses werd de generaliseerbaarheid van de aannames bepaald. Zowel de kosten van gezondheidszorg als de kosten voor patiënt en familie waren vergelijkbaar tussen beide groepen. Aangezien het programma geen effect bleek te hebben op het aantal valincidenten, het dagelijks functioneren of de kwaliteit van leven, werd geconcludeerd dat het programma niet kosteneffectief is vergeleken met reguliere zorg in Nederland.

Hoofdstuk 7 doet verslag van een studie met als doel discontinue metingen van de kosten van gezondheidszorggebruik te vergelijken met continue metingen. Continu meten van gezondheidszorggebruik vormt vaak een belasting voor de patiënt, met als gevolg ontbrekende waarden of uitval van deelnemers. Daarom is het belangrijk om te onderzoeken wat de beste manier is om de jaarlijkse gezondheidszorgkosten te schatten op basis van kortere meetperiodes. In deze studie hebben we daarom de kosten zoals die continu waren gemeten over een heel jaar, vergeleken met gesimuleerde kosten over een heel jaar die waren verkregen met behulp van verschillende discontinue meetpatronen, gecombineerd met drie veel gebruikte imputatietechnieken: individueel gemiddelde, "Last Observation Carried Forward", en "Next Observation Carried Backward". De analyses bevestigden dat discontinu meten met kostendagboekjes goede schattingen oplevert van het gezondheidszorggebruik over een heel jaar. Wel bleek dat het meetpatroon en de gebruikte imputatietechniek van invloed waren op de uitkomsten. De beste benadering van het gezondheidszorggebruik over een heel jaar op basis van discontinue metingen bleek het gebruik van drie random cohorten, waarbij elke maand bij tenminste een derde van de deelnemers wordt gemeten, gecombineerd met imputatie via het individuele gemiddelde. 
Hoofdstuk 8 vat de voornaamste bevindingen van de voorafgaande hoofdstukken samen en presenteert methodologische en theoretische overwegingen. Ook worden implicaties en aanbevelingen voor praktijk, beleid en verder onderzoek aangegeven. De belangrijkste conclusie is dat het onderzochte medisch-ergotherapeutische programma weliswaar praktisch uitvoerbaar bleek, maar niet (kosten-)effectief is vergeleken met de reguliere zorg in Nederland. Niettemin blijft de impact van vallen op de maatschappij en het individu groot. Daarom is onze aanbeveling om dit programma niet in de huidige vorm in te voeren, maar bepaalde aspecten te verbeteren en te onderzoeken of deze aanpassingen leiden tot een grotere effectiviteit en efficiëntie. We denken daarbij aan de volgende aanpassingen: betere selectie van mensen met een verhoogd valrisico, het sneller uitvoeren van de preventiemaatregelen, en het toevoegen van beweeg- en oefenprogramma's. Daarnaast geven we enkele aanbevelingen voor vervolgonderzoek. 


\section{CO-AUTHORS \& AFFILIATIONS}

Michel HC Bleijlevens, MSc

Maastricht University Department of Social Medicine, Faculty of Health, Medicine and Life Sciences; School for Public Health and Primary Care (Caphri), Maastricht, the Netherlands

Fleur de Bruijn, MD

Maastricht University Department of General Practice, Faculty of Health, Medicine and Life Sciences, Maastricht, the Netherlands

Harry FJM Crebolder, MD, PhD

Maastricht University, Department of General Practice, Faculty of Health, Medicine and Life Sciences; School for Public Health and Primary Care (Caphri), Maastricht, the Netherlands

Joseph PM Diederiks, PhD

School for Public Health and Primary Care (Caphri), Maastricht, The Netherlands

Jacques ThM van Eijk, PhD

Department of Social Medicine, Faculty of Health, Medicine and Life Sciences Maastricht University; School for Public Health and Primary Care (Caphri), Maastricht, the Netherlands

Silvia MAA Evers, PhD

Maastricht University, Department of Health Organization, Policy and Economics, Faculty of Health, Medicine and Life Sciences; School for Public Health and Primary Care (Caphri), Maastricht, the Netherlands

Jolanda CM van Haastregt, PhD

Maastricht University, Department of Health Care and Nursing Science, Faculty of Health, Medicine and Life Sciences; School for Public Health and Primary Care (Caphri), Maastricht, the Netherlands

Gertrudis IJM Kempen, PhD

Maastricht University, Department of Health Care and Nursing Science, Faculty of Health, Medicine and Life Sciences; School for Public Health and Primary Care (Caphri), Maastricht, the Netherlands 
Wubbo J Mulder, MD, PhD

Maastricht University Hospital, Department of Internal Medicine, Division Medicine and Geriatrics, Maastricht, the Netherlands

Erik van Rossum, PhD

Maastricht University, Department of Health Care and Nursing Science, Faculty of Health, Medicine and Life Sciences; School for Public Health and Primary Care (Caphri), Maastricht; Professional University Zuyd, Faculty of Health and Technology, Heerlen, the Netherlands

Joop M Ruijgrok, MD, PhD

Maastricht University Hospital, Department of Internal Medicine, Division Medicine and Geriatrics, Maastricht, the Netherlands

Paul A Stalenhoef,MD, PhD

Maastricht University, Department of General Practice, Faculty of Health, Medicine and Life Sciences; School for Public Health and Primary Care (Caphri), Maastricht, the Netherlands 


\section{DANK！}

Dit proefschrift was nooit tot stand gekomen zonder de betrokkenheid en ondersteuning van veel mensen en organisaties. Hierbij wil ik graag iedereen bedanken die daaraan heeft bijgedragen. Een aantal mensen wil ik daarbij in het bijzonder noemen.

Allereerst dank aan alle deelnemers van het onderzoek en de pilotstudie. Zonder jullie deelname was het niet mogelijk geweest.

In het bijzonder gaat mijn dank uit naar mijn promotoren en copromotoren Harry Crebolder, Jacques van Eijk, Jolanda van Haastregt en Silvia Evers. Samen vormden jullie voor mij een team waarin ik van alle kanten werd uitgedaagd om het beste resultaat naar boven te halen, bedankt daarvoor! Harry, bedankt voor de het vertrouwen dat je me hebt gegeven, en de positieve woorden op momenten dat het voor mij soms allemaal onmogelijk leek. Jouw eeuwig enthousiasme heeft me enorm gemotiveerd! Jacques, bedankt dat je zoals je zelf vaak zei 'advocaat van de duivel' speelde, kritische vragen stelde, me stimuleerde mijn ideeën helder te formuleren en onderbouwen. Ook niet te vergeten: je waarschuwingen om niet te veel de diepte in te duiken, met het gevaar de rode draad te verliezen. Hiervan heb ik veel geleerd. Jolanda, eigenlijk moet ik zeggen mede mogelijk gemaakt door JvH. Bedankt dat jij jouw project aan mij hebt toevertrouwd. Misschien dat je daarom vaak mijn eerste aanspreekpunt was, maar daardoor heb ik geweldig veel van je geleerd. Je hebt me enorm gemotiveerd en vertrouwen gegeven. Bedankt dat ik altijd bij je kon binnenlopen, ik vind het fijn dat jij dat tegenwoordig ook bij mij kan doen! En last but not least Silvia, als laatste sloot jij voor de HTA ondersteuning bij het team aan. Door jouw enthousiasme en ondersteuning heb ik me ook op dat gebied kunnen ontwikkelen. Je bent een onuitputtelijke bron van ideeën en hebt me enorm geïnspireerd. Samen hebben we de enorme HTA-data-berg bedwongen, en daarna werd het alleen maar interessanter!

De leden van de beoordelingscommissie, te weten voorzitter prof.dr. J.L. Severens, prof.dr. J.M.G.A. Schols, prof.dr. J.A. Knottnerus, prof.dr.ir. H.C.W. de Vet, prof.dr. H.J.J. Verhaar, wil ik van harte bedanken voor het beoordelen van dit proefschrift. 
De leden van de projectgroep en/of coauteurs: Jos Diederiks, Ruud Kempen, Erik van Rossum, Paul Stalenhoef, Peter Brink, Luc de Witte. Hoewel het aantal verschillende meningen met zo'n grote groep mensen toeneemt, was het team van alle markten thuis. Voor elke vraag kon ik wel bij iemand terecht, bedankt voor jullie adviezen. Daarnaast gaat mijn dank uit naar de uitvoerders van de interventie: Wubbo Mulder, Joop Ruygrok, Ludo Scheres, Ron Warnier, Tanja de Bie, Charlotte Mom en Evelien Cratzborn. Dat het project zo goed werd ontvangen door de deelnemers is mede dankzij jullie inzet!

Tevens wil ik alle betrokken organisaties bedanken. Huize Blankenberg bedankt dat we de pilot studie op deze prachtige lokatie konden uitvoeren. Het Azm, in het bijzonder de afdelingen Spoed Eisende Hulp en Radiologie en Huisartsenpost Maastricht heel erg bedankt dat we wekelijks mochten inbreken om de nieuwe 'gevallen' uit jullie administratie te vissen. Het Memic, bedankt: Jack Berben als proefkonijn van de rolodex, ging het niet altijd in 1x goed, maar we hebben we enorm veel gehad aan deze logistieke ondersteuning, Annemie Mordant, Hilde Sielhorst, Marlene Ronner bedankt voor jullie hulp en tips. Ook alle enquêtrices bedankt! Sommige deelnemers gaven aan jullie telefoontjes zelfs te missen. Daarnaast gaat mijn dank uit naar de medewerkers van het Regionaal Indicatie Orgaan Maastricht, Gemeente Maastricht, Gemeente Valkenburg, Gemeente Meerssen, Gemeente Magraten en Gemeente Eijsden, bedankt voor de medewerking bij onder andere de uitvoering van de WVG.

Mijn (oud)collega's bij Medische Sociologie wil ik bedanken voor de gezelligheid en kritische reflectie tijdens de 'THEE!', AIO-soep of andere momenten. Mijn nieuwe collega's bij zowel BEOZ als KEMTA en het TTOPBCCproject wil ik bedanken voor hun hartelijke ontvangst. Ik heb heel veel zin er nu echt met volle kracht tegen aan te gaan!

Dank ook aan al mijn intervalproject-genoten (ja ja verleden tijd van genieten). Fleur, ik ben blij dat ik samen met jou gestart ben. We hebben heel veel gelachen en gezweet (en niet alleen op de fiets naar Blankenberg). Ik vond het echt heel jammer dat je stopte, maar enorm bedankt voor het vertrouwen dat je me hebt gegeven om het project af te ronden. En nu zijn we toch allebei dr! Natasja en Violet, fijn dat jullie zo snel op die rijdende trein zijn gesprongen, uiteindelijk hebben jullie beide gekozen voor een ander spoor. En toen 
kwam er eindelijk een echte vent. Michel, misschien was het wel omdat we niet fulltime met elkaar zaten opgescheept;-), maar het is nog steeds genieten! We hebben inmiddels onze eigen interval taal ontwikkeld, en blikken zeggen genoeg. Gelukkig maar, want we hebben het soms beide te druk om nóg meer te ouwehoeren. Ik ben blij dat jij naast me staat op deze dag, en de volgende keer sta jij in het midden!

Dank ook aan alle lieve mensen om me heen, dicht bij en ver weg. Zoveel lieve vrienden die me waarschuwden dat ik niet te veel hooi op mijn vork zou moeten nemen, en er toch weer voor me waren als ik zelf ontdekte dat het misschien toch allemaal wel een beetje veel was. Wie niet waagt wie niet wint, maar zonder jullie eindeloze geduld, pep-talks, begrip, telefoontjes, berichtjes, etentjes, koffiebreaks, theemomenten, afleidende bike- en klimtripjes en wat al niet meer, was dit alles me nooit gelukt! Ik vind het fijn om er nu weer meer voor jullie te zijn!

Michelle, lieve zus, op eigen wijze heb jij me gesteund al die jaren, met je enorme relativeringsvermogen, je daadkracht (op het moment dat ik ervaringsdeskundige werd kwam je gewoon een week 'professionele thuiszorg' bieden!), je kaartjes, krabbels, telefoontjes, samen zijn en niet te vergeten 'Sint's grote INSPIRATIE boek aflevering 2007 - valpreventie bij ouderen'. Ik ben super trots dat jij naast me staat op deze bijzondere dag.

Tot slot, lieve papa en mama, wie had ooit gedacht dat ik hier zou staan? Jullie misschien wel, maar het is zeker dankzij jullie oneindige interesse en vertrouwen in mij. Jullie hebben me enorm gesteund, door er altijd te zijn, op welke manier dan ook, niks is jullie te veel. Dank jullie wel.
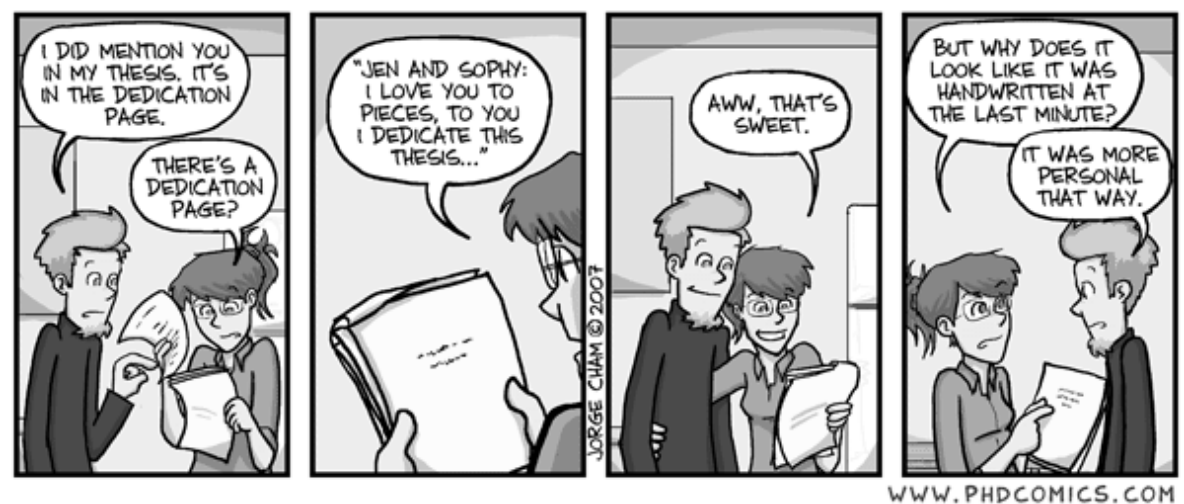


\section{CURRICULUM VITAE}

Marike Hendriks was born in Delft (the Netherlands) on March 25, 1977. She attended secondary school (VWO) at the Onze Lieve Vrouwe Lyceum in Breda. After her graduation in 1995 she studied Health Sciences at Maastricht University. She completed her Masters in Movement Science and Health Education and Health Promotion in 2001. In 2002 she started as a researcher on a fall prevention project at the Faculty of Health Medicine and Life Sciences, and the School for Public Health and Primary Care (CAPHRI), both part of Maastricht University. The project included a longitudinal randomized controlled trial to assess the effectiveness and cost-effectiveness of a multidisciplinary fall prevention programme aimed at community-living elderly people at risk for falling. Health Technology Assessment was also part of the project. From June 2006 until March 2008 she combined her PhD research with several projects in the field of social care at the School for Public Health and Primary Care (CAPHRI). In December 2007 she started as assistant professor Strategic Management at the department of Health Organisation Policy and Economics (HOPE), at Maastricht University. Since March 2008 she also works as a researcher at the Department of Clinical Epidemiology and Medical Technology Assessment (KEMTA) at the University Hospital Maastricht, by joining a project on three non-invasive treatment options for superficial basal cell carcinoma (cost minimalisation analysis and a discrete choice experiment). 